\title{
Thermal Production of Gravitinos
}

\author{
Dissertation \\ zur Erlangung des Doktorgrades \\ des Fachbereichs Physik \\ der Universität Hamburg
}

vorgelegt von

Matthias Bolz

aus Heilbronn

Hamburg

1999 
Gutachter der Dissertation:

Gutachter der Disputation:

Datum der Disputation:

Dekan des Fachbereichs Physik und Vorsitzender des

Promotionsausschusses:
Prof. Dr. Wilfried Buchmüller

Prof. Dr. Joachim Bartels

Prof. Dr. Wilfried Buchmüller

Prof. Dr. Bernd A. Kniehl

21. März 2000

Prof. Dr. Friedrich-Wilhelm Büßer 


\begin{abstract}
The gravitino regeneration rate $\Gamma_{\tilde{G}}$ is calculated perturbatively to the leading logarithmic and corresponding constant order in the strong coupling constant $g$. Two approaches of obtaining the coefficient of the leading logarithmic term, one starting from individual gravitino production processes and one applying the thermal version of the optical theorem, are shown to deliver analytically the same result. In the second approach the next-to-leading constant term is obtained along with that coefficient. Here the imaginary part of the gravitino self energy is calculated in the framework of hard thermal loop effective field theory, with a resummed gluon propagator for both soft and hard gluons. The constant is strongly depending on the gravitino energy $E$. As an application, the implications of a large baryogenesis temperature, $T_{B}=\mathcal{O}\left(10^{10} \mathrm{GeV}\right)$, on the mass spectrum of superparticles have been re-analyzed with the new value for $\Gamma_{\tilde{G}}$. Previous phenomenological conclusions remain essentially unchanged.
\end{abstract}

\title{
Zusammenfassung
}

Die Gravitino-Regenerationsrate $\Gamma_{\tilde{G}}$ wurde störungstheoretisch zu führender logarithmischer und zu dazugehöriger konstanter Ordnung in der starken Kopplungskonstanten $g$ berechnet. Zwei verschiedene Methoden, den Koeffizienten des führenden logarithmischen Terms zu berechnen - die eine geht von einzelnen Gravitino-Erzeugungsprozessen aus, während die andere das optische Theorem bei endlicher Temperatur benutzt - liefern analytisch dasselbe Ergebnis. Mit der zweiten Methode erhält man darüberhinaus den nächstführenden konstanten Term: Der Imaginärteil der Gravitino-Selbstenergie wurde im Rahmen der effektiven "hard thermal loop"-Feldtheorie berechnet, wobei sowohl für weiche als auch für harte Gluonen ein resummierter Gluonpropagator verwendet wurde. Die Konstante zeigt eine starke Abhängigkeit von der Gravitino-Energie E. Als Anwendung des neuen Werts für $\Gamma_{\tilde{G}}$ wurde erneut untersucht, welche Konsequenzen eine hohe Baryogenesetemperatur, $T_{B}=\mathcal{O}\left(10^{10} \mathrm{GeV}\right)$, auf das Massenspektrum supersymmetrischer Teilchen hat. Die Ergebnisse einer früheren Untersuchung behalten im wesentlichen ihre Gültigkeit. 

Denn, was man schwarz auf weiß besitzt, Kann man getrost nach Hause tragen.

J. W. v. Goethe, Faust 



\section{Contents}

$\begin{array}{ll}\text { Introduction } & 1\end{array}$

1 Spin-3/2 Particles 5

1.1 Equation of motion ..................... . . . . . . . . . .

1.2 Wave function and spin sum . . . . . . . . . . . . . . 6

1.3 Spin sum for states with maximal helicity . . . . . . . . . . . . 8

1.4 Effective theory for light gravitinos . . . . . . . . . . . . . . 11

2 Gravitino Production Processes 13

2.1 Example of a $2 \rightarrow 2$ cross section for gravitino production . . . . . . . . . 13

2.2 Summary of all ten $2 \rightarrow 2$ cross sections . . . . . . . . . . . . . . 17

2.3 Two cut-off methods . . . . . . . . . . . . . . . . . . . . 20

2.4 The Boltzmann collision term . . . . . . . . . . . . . . 22

3 The Gravitino Self Energy $\quad 27$

3.1 The optical theorem for finite temperature . . . . . . . . . . . . . . 27

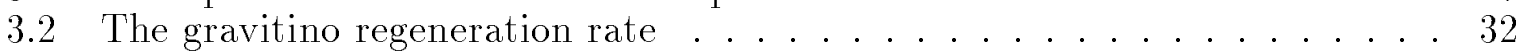

3.2.1 Soft loop momenta . . . . . . . . . . . . . . . . . . . . . 39

3.2.2 Hard loop momenta. . . . . . . . . . . . . . . . . . . . . 40

3.2.3 The Boltzmann collision term ................ 44

4 Baryon Asymmetry And Dark Matter $\quad 46$

4.1 Baryogenesis and the gravitino problem . . . . . . . . . . . . 47

4.2 The gravitino abundance after inflation . . . . . . . . . . . . . . 47

4.3 An inflationary scenario with $T_{R} \simeq T_{B} \simeq \mathcal{O}\left(10^{10} \mathrm{GeV}\right) \ldots . . . . . .49$

$\begin{array}{ll}\text { Conclusions } & 53\end{array}$

A Feynman Rules $\quad \mathbf{5 5}$

A.1 External particle lines . . . . . . . . . . . . . . . . 55

A.2 Propagators . . . . . . . . . . . . . . . . . . . . 56

A.3 Vertices . . . . . . . . . . . . . . . . . 56

A.3.1 Vertices of SUSY-QCD . . . . . . . . . . . . 57

A.3.2 Vertices with gravitinos (full theory) . . . . . . . . . . . . 58

A.3.3 Vertices with gravitinos (effective theory for light gravitinos) . . . . 60

B Evaluation of The Phase Space Integrals in The Laboratory System 62

B.1 General formalism . . . . . . . . . . . . . . . . 62

B.2 Integration of a divergent differential cross section $\propto \frac{1}{t} \ldots \ldots \ldots$ 
C The Supersymmetric Effective Gluon Mass

D Approximations in The Calculation of The Imaginary Part of The Gravitino Self Energy

D.1 Contribution of the pole terms to the imaginary part of the gravitino self energy . . . . . . . . . . . . . . . . . . . . . 69

D.2 Approximation of the $\omega$ integration limits $\ldots \ldots \ldots \ldots \ldots$ 


\section{Introduction}

Supergravity is the theory of supersymmetry extended from a global to a local symmetry. A fundamental ingredient of supergravity is the gravitino field. The gravitino is the gauge field of supersymmetry. It is a spin-3/2 field and the supersymmetric partner of the graviton [1]. As long as supersymmetry prevails, it only couples with gravitational strength $\sim \frac{1}{M}$ (where $M=\left(8 \pi G_{\mathrm{N}}\right)^{-\frac{1}{2}} \simeq 2.4 \cdot 10^{18} \mathrm{GeV}$ is the Planck mass) to other fields, and it is massless, just as the graviton.

When SUSY is broken, two things change. First, the gravitino acquires mass trough the super Higgs mechanism [2], eating the goldstino degrees of freedom. In a supersymmetric theory, goldstinos are the fermions corresponding to the Goldstone bosons arising from global symmetry breaking. Second, the gravitino's coupling strength increases from the gravitational strength $\sim \frac{1}{M}$ to a strength which can be as strong as a weak-type interaction [3]. Both phenomena, the mass and the increased coupling, are related: the gravitino mass $m_{\tilde{G}}$ is of the order of $[3]$

$$
m_{\tilde{G}} \sim \frac{\Lambda^{2}}{M}
$$

where $\Lambda$ is the scale of SUSY breaking. In turn, the gravitino's interaction strength after SUSY breaking is enhanced by a factor of scale of SUSY breaking over gravitino mass. Even with this enhanced interaction strength, however, gravitinos play a particular role in the spectrum of supergravity particles: they are a massive species which decouples much earlier than all other massive particles from thermal equilibrium (except for the case of extremely light gravitinos), resulting in a very high gravitino abundance in the universe. This has been used to derive cosmological constraints. In [4] an upper mass bound of $m_{\tilde{G}}<1 \mathrm{keV}$ on stable gravitinos was derived from the requirement that overclosure of the universe has to be avoided. The authors of $[5,6]$ found a lower mass bound $m_{\tilde{G}}>\mathcal{O}\left(10^{4}\right) \mathrm{GeV}$ on unstable gravitinos. If unstable gravitinos were lighter, the enormous amount of entropy from their decay would be produced late enough to disturb nucleosynthesis.

Inflation may be a way to avoid these gravitino problems and to reopen the window for 
a more natural gravitino mass range between several $\mathrm{MeV}$ and several hundred $\mathrm{GeV}$ [7]. In an inflationary epoch, any primordial gravitino abundance is enormously diluted. The cosmologically relevant gravitino abundance is created in the reheating phase after the inflationary period. This reheating period is characterized by the maximum temperature reached, the reheating temperature $T_{R}$. Gravitinos are dominantly produced by inelastic $2 \rightarrow 2$ scattering processes of particles from the thermal bath [8]. The gravitino abundance is essentially linear in the reheating temperature $T_{R}$. If one chooses $T_{R}$ too large, one encounters again the old gravitino problems of a possible overclosure of the universe or a disturbance of nucleosynthesis. This, in turn, poses constraints on baryogenesis models that may require a large reheating temperature, such as baryogenesis through leptogenesis $[9]$.

Thus in [8] a bound $T_{R}<10^{8}-10^{12} \mathrm{GeV}$ for the reheating temperature was found. The authors of [10] were the first ones to calculate the production cross sections for all relevant inelastic $2 \rightarrow 2$ production processes for gravitinos heavier than gauginos. They derived a bound of $T_{R}<10^{9}-10^{10} \mathrm{GeV}$. Four of the ten production processes turned out to have a logarithmically singular cross section due to the exchange of infrared massless gluons. The divergence was regularized by an ad hoc introduced effective thermal gluon mass. The analysis of [10] was refined and extended to the case of light gravitinos by [11]; a bound $T_{R}<10^{2}-10^{10} \mathrm{GeV}$, as a function of $m_{\tilde{G}}$, was derived there.

In 1994, Fischler [12] claimed a much higher production rate than the authors before him had found. Due to heat-bath effects the gravitino regeneration rate should be proportional to $T^{5}$, instead of $T^{3}$, as estimated previously. If correct, this would have dramatic consequences both for cosmology and supersymmetric phenomenology.

Motivated by Fischler's considerations, Ellis et al. [13] studied the leading finitetemperature effects of the gravitino regeneration rate in the effective theory for light gravitinos. In a new approach the gravitino regeneration rate was calculated from the imaginary part of the gravitino self energy rather than from considering individual gravitino production processes. The old result that the gravitino regeneration rate is proportional to $T^{3}$ could be confirmed, Fischler's argument was not supported. Furthermore, in [13] it became apparent that the divergent cross sections are regularized by the electric rather than the magnetic Debye mass of the gluon, and that the logarithm which arose from the cut-off procedure actually is the leading term in an expansion of the gravitino regeneration rate in powers of the strong coupling constant. However, the coefficients of this leading logarithmic term found in both approaches differed by a factor of $\sim 15$, and the constant term remained unknown, because only the contribution from soft gluons was calculated. 
In [14] we generalized the calculation of the ten dominant gravitino production processes to arbitrary gravitino masses. It turned out that adding the results of the previously considered limiting cases of very light and very heavy gravitinos yields the general result, with no new contributions from the intermediate gravitino mass range. Furthermore, we found an inflationary scenario with a large reheating temperature $T_{R}=\mathcal{O}\left(10^{10} \mathrm{GeV}\right)$ which is consistent with the gravitino constraints.

This thesis presents two new results: the constant term of the gravitino regeneration rate $\Gamma_{\tilde{G}}$ is calculated for the first time consistently in the framework of thermal field theory, and the coefficient of the leading logarithmic term is determined with improved accuracy. The integral over $\Gamma_{\tilde{G}}$, weighted with a Fermi distribution, leads to the Boltzmann collision $\operatorname{term} \mathcal{C}_{\tilde{G}}$ of the Boltzmann equation for the time evolution of the gravitino number density. It is demonstrated that the coefficients of the leading logarithmic term of $\mathcal{C}_{\tilde{G}}$ found in the two approaches coincide analytically. As an application of these new results, the validity of our large $T_{R}$ scenario [14] could be confirmed. The gravitino constraints are even slightly relaxed, but this effect is overcompensated by using a smaller Hubble parameter $h$ compared to [14].

The constant in the gravitino regeneration rate is found by extending the calculation of [13] to include also the contribution from hard gluons. Since the logarithm of the strong coupling is numerically quite small (about a third of the constant term), knowing the constant term means a considerable improvement for any quantitative discussion of the gravitino problem.

The improved accuracy in the coefficient of the leading logarithmic term is realized by including a quantum statistical factor for the particle which is produced along with the gravitino in the $2 \rightarrow 2$ gravitino production processes when calculating the Boltzmann collision term $\mathcal{C}_{\tilde{G}}$. The inclusion of the quantum statistical factor, which is usually set to 1 in this approach, is possible by doing all necessary phase space integrations in the laboratory system. The result for the coefficient of the leading logarithmic term coincides analytically with the one found by [13] in the self energy approach, if one supplies two numerical factors which have been overlooked in [13]. This provides a consistency check for the two approaches.

In the recent past, several publications appeared in which non-thermal production of gravitinos was studied. In [15] for example an investigation of gravitino production during preheating due to a combined effect of interactions with an oscillating inflaton field and absence of conformal invariance is carried out. Although non-thermal mechanisms can lead to a gravitino production much more copious than thermal processes, the efficiency of these new non-thermal mechanisms is very sensitive to the choice of the underlying 
inflation model. In this thesis, I do not consider such non-thermal mechanisms but only the thermal production of gravitinos which is always present, independent of the specific inflation model.

The thesis has four chapters. In chapter 1 the theory of massive and massless spin- $3 / 2$ particles is reviewed and it is shown how to calculate processes that involve such particles. In chapter 2 the cross sections of the ten dominant gravitino production processes are calculated. From these cross sections the Boltzmann collision term governing the Boltzmann equation for the time development of the gravitino number density is calculated. In chapter 3 a different approach is used to calculate the same Boltzmann collision term: using the thermal version of the optical theorem, it is obtained from the imaginary part of the gravitino self energy. In chapter 4 an update of our analysis [14] is presented, where the implications of a large reheating temperature, $T_{R}=\mathcal{O}\left(10^{10} \mathrm{GeV}\right)$, on the mass spectrum of superparticles was studied. The result is that the phenomenological conclusions remain essentially unchanged.

Appendix A contains the Feynman rules of SUSY QCD with gravitinos. In appendix B the technical details are explained of how to perform all phase space integrations of the Boltzmann collision term in the laboratory system. This is needed in chapter 2 in order to keep the quantum statistical factor for the particle which is produced along with the gravitino in a $2 \rightarrow 2$ process. In appendix $C$ the supersymmetric effective thermal gluon mass is calculated. In appendix D the approximations made when calculating the imaginary part of the gravitino self energy in chapter 3 are justified. 


\section{Chapter 1}

\section{Spin-3/2 Particles}

The objective of this chapter is to provide both the theoretical background of quantized spin-3/2 fields and the tools to do practical calculations with massive and massless spin$3 / 2$ particles. I start with deriving the equation of motion for the Rarita-Schwinger field. The wave function, the spinor vector $\psi_{\mu}(P)$, is derived, as well as the expression for the sum $\Pi_{\mu \nu}(P)$ over projection operators on all four helicity states. The wave functions are

then used to construct explicitly the sum $\Pi_{\mu \nu}^{\frac{3}{2}}(P)$ over projection operators on the massless states with helicity $+3 / 2$ and $-3 / 2$, and it is shown how to do practical calculations with $\Pi_{\mu \nu}^{\frac{3}{2}}(P)$. Finally the effective theory for light gravitinos is explained. In this theory only the spin-1/2 or goldstino components of the gravitino are left over, and the gravitino can simply be treated as an ordinary spin-1/2 particle.

\subsection{Equation of motion}

The field equations for spin-3/2 particles are a particular case of the Bargmann-Wigner equations $[16,17]$ which are a system of relativistic wave equations for arbitrary spin. In the Bargman-Wigner formulation, a field of rest mass $m$ and spin $s \geq \frac{1}{2}$ is represented by a completely symmetric multispinor $\psi^{\alpha \beta \gamma \ldots \tau}(x)$ of rank $2 s$ satisfying Dirac-type equations in all indices:

$$
\begin{aligned}
& (i \not \partial-m)^{\alpha \alpha^{\prime}} \psi^{\alpha^{\prime} \beta \gamma \ldots \tau}(x)=0 \\
& (i \not \partial-m)^{\beta \beta^{\prime}} \psi^{\alpha \beta^{\prime} \gamma \ldots \tau}(x)=0
\end{aligned}
$$

In the case of $s=\frac{3}{2}$ one can show that the symmetric rank 3 spinor $\psi^{\alpha \beta \gamma}(x)$ is uniquely 
determined by the components of a vector-spinor $\psi_{\mu}^{\alpha}(x)$. This vector-spinor is a more convenient object to deal with as a wave function than the multispinor $\psi^{\alpha \beta \gamma}(x)$. $\psi_{\mu}^{\alpha}(x)$ carries both a Dirac spinor index $\alpha$ and a Lorentz vector index $\mu$; the Dirac spinor index will be suppressed in the following text. From the symmetry requirement in all three spinor indices and from the Bargman-Wigner equations 1.2 for $\psi^{\alpha \beta \gamma}(x)$ one derives for $\psi_{\mu}(x)$ the two equations of motion

$$
\begin{aligned}
\gamma^{\mu} \psi_{\mu}(x) & =0, \\
(i \not \partial-m) \psi_{\mu}(x) & =0,
\end{aligned}
$$

which are know as the Rarita-Schwinger equations [18]. They yield the further constraint

$$
\partial^{\mu} \psi_{\mu}(x)=0
$$

An alternative way to derive the Rarita-Schwinger equations is to start from the Lagrangian density for a free spin-3/2 field:

$$
\mathcal{L}=-\frac{1}{2} \varepsilon^{\mu \nu \rho \sigma} \bar{\psi}_{\mu} \gamma_{5} \gamma_{\nu} \partial_{\rho} \psi_{\sigma}-\frac{1}{4} m \bar{\psi}_{\mu}\left[\gamma^{\mu}, \gamma^{\nu}\right] \psi_{\nu}
$$

This density is part of the full supergravity Lagrangian [19], describing the motion of a free gravitino. Varying eq. 1.5 with respect to $\psi_{\mu}$ yields

$$
0=\frac{\partial \mathcal{L}}{\partial \bar{\psi}_{\mu}}-\partial_{\rho} \frac{\partial \mathcal{L}}{\partial\left(\partial_{\rho} \bar{\psi}_{\mu}\right)}=-\frac{1}{2} \varepsilon^{\mu \nu \rho \sigma} \gamma_{5} \gamma_{\nu} \partial_{\rho} \psi_{\sigma}-\frac{1}{4} m\left[\gamma^{\mu}, \gamma^{\nu}\right] \psi_{\nu}
$$

With the identity

$$
\gamma_{\mu} \varepsilon^{\mu \alpha \beta \gamma}=-i \gamma_{5}\left(\gamma^{\alpha} \gamma^{\beta} \gamma^{\gamma}-\gamma^{\alpha} g^{\beta \gamma}+\gamma^{\beta} g^{\alpha \gamma}-\gamma^{\gamma} g^{\alpha \beta}\right)
$$

this also leads to the Rarita-Schwinger equations 1.2 and 1.3.

\subsection{Wave function and spin sum}

Following [20], the solutions $\psi_{\mu}^{l}(P)$ to the Rarita-Schwinger equations 1.2 and 1.3 in momentum space with helicity index $l=+\frac{3}{2},+\frac{1}{2},-\frac{1}{2},-\frac{3}{2}$ can be constructed as direct products of ordinary Dirac spinors $u^{s}(P), v^{s}(P)\left(s=+\frac{1}{2},-\frac{1}{2}\right)$ for massive spin-1/2 particles and polarization vectors $\varepsilon_{\mu}^{m}(P)(m=+1,0,-1)$ for massive spin-1 particles: 


$$
\begin{aligned}
& \psi_{\mu}^{l+}(P)=\sum_{s, m} C(l, s, m) u^{s}(P) \varepsilon_{\mu}^{m}, \\
& \psi_{\mu}^{l-}(P)=\sum_{s, m} C(l, s, m) v^{s}(P) \varepsilon_{\mu}^{m},
\end{aligned}
$$

where the $C(l, s, m)$ are Clebsch-Gordon coefficients. Since the gravitino is a Majorana particle for which the charge conjugated wave function equals the wave function itself, I will restrict myself to positive energy solutions $\psi_{\mu}^{l+}(P)$ involving only $u^{s}(P)$ and drop the superscript + in $\psi_{\mu}^{l+}(P)$.

The Dirac spinors are normalized to

$$
\bar{u}^{s} u^{s^{\prime}}=2 m \delta^{s s^{\prime}}
$$

and for the polarization vectors one has

$$
\varepsilon_{\mu}^{m} \varepsilon^{m^{\prime} * \mu}=-\delta^{m m^{\prime}},
$$

therefore the wave functions $\psi_{\mu}^{l}(P)$ are normalized to

$$
\bar{\psi}_{\mu}^{l} \psi^{l^{\prime} \mu}=-2 m \delta^{l l^{\prime}}
$$

The sum $\Pi_{\mu \nu}(P)=\sum_{l} \psi_{\mu}^{l}(P) \bar{\psi}_{\nu}^{l}(P)$ of projection operators on all four helicity states can be obtained as follows [17]: Because of Lorentz invariance, $\Pi_{\mu \nu}(P)$ must be a linear combination of the ten tensors

$$
\begin{array}{lll}
m g_{\mu \nu}, & m \gamma_{\mu} \gamma_{\nu}, \quad \gamma_{\mu} P_{\nu}, \quad \gamma_{\nu} P_{\mu}, \quad \frac{P_{\mu} P_{\nu}}{m} \\
\not P g_{\mu \nu}, & P P \gamma_{\mu} \gamma_{\nu}, \quad \not P \gamma_{\mu} \frac{P_{\nu}}{m}, \quad \not P \gamma_{\nu} \frac{P_{\mu}}{m}, \quad \not P \frac{P_{\mu} P_{\nu}}{m^{2}}
\end{array}
$$

From the requirement that $\Pi_{\mu \nu}(P)$ must satisfy the Rarita-Schwinger equations

$$
\begin{aligned}
\gamma^{\mu} \Pi_{\mu \nu}(P) & =0, \\
(\not P-m) \Pi_{\mu \nu}(P) & =0,
\end{aligned}
$$

and must have the projector property

$$
\Pi_{\mu}^{\lambda}(P) \Pi_{\lambda \nu}(P)=-2 m \Pi_{\mu \nu}(P),
$$

the coefficients of the ten terms can be determined in a unique manner. The result is that all ten terms 1.13 figure in $\Pi_{\mu \nu}(P)$,

$$
\Pi_{\mu \nu}(P)=-(\not P+m)\left[g_{\mu \nu}-\frac{P_{\mu} P_{\nu}}{m^{2}}-\frac{1}{3}\left(g_{\mu \rho}-\frac{P_{\mu} P_{\rho}}{m^{2}}\right)\left(g_{\nu \sigma}-\frac{P_{\nu} P_{\sigma}}{m^{2}}\right) \gamma^{\rho} \gamma^{\sigma}\right]
$$




\subsection{Spin sum for states with maximal helicity}

In this section the sum $\Pi_{\mu \nu}^{\frac{3}{2}}(P)$ of projection operators on states with helicity $+3 / 2$ and $-3 / 2$ is constructed explicitly. In the limit of very heavy gravitinos, only the gravitino components with maximal helicity play a role. $\Pi_{\mu \nu}^{\frac{3}{2}}(P)$ is needed if one wants to calculate just their contribution to a process.

Helicity is the spin projection on the momentum axis. For massive particles this is not a Lorentz invariant quantity. Therefore it is not possible to extract in a Lorentz invariant manner the contributions of single helicity states to a complete cross section. However, one can go to the case of massless spin-3/2 particles. In this case, helicity becomes equal to chirality, which is a Lorentz invariant quantity. Now one can even isolate the two spin orientations + and - .

I start by constructing the wave functions $\psi_{\mu}^{+}=\psi_{\mu}^{+\frac{3}{2}}$ and $\psi_{\mu}^{-}=\psi_{\mu}^{-\frac{3}{2}}$ according to eq. 1.9 extended to the case of massless particles. To this end I need two Dirac spinors $u^{+}(P)$ and $u^{-}(P)$ of a massless spin-1/2 particle with four momentum $P$ and the three polarization vectors $\varepsilon^{+}, \varepsilon^{0}$, and $\varepsilon^{-}$of a massless spin-1 particle.

The Dirac spinors satisfy the massless Dirac equation

$$
\not P u^{ \pm}(P)=0
$$

and are eigenstates of the chirality operator:

$$
\gamma_{5} u^{ \pm}= \pm u^{ \pm}
$$

Massless spinors cannot be normalized in the same way as massive ones (eq. 1.10); instead one has

$$
\bar{u}^{s}(P) \gamma^{\mu} u^{s^{\prime}}(P)=2 P^{\mu} \delta^{s s^{\prime}}
$$

The spinors yield the projection operators

$$
u^{ \pm}(P) \bar{u}^{ \pm}(P)=\left(1 \pm \gamma_{5}\right) \frac{\not P}{2}
$$

To construct the polarization vectors $\varepsilon_{\mu}^{m}$ one introduces a time axis $n, n^{2}=1, n^{0}>0$. The vector of longitudinal polarization is then

$$
\varepsilon^{(3)}=\frac{1}{n \cdot P}[P-(n \cdot P) n]
$$

Next one chooses a transverse polarization vector $\varepsilon^{(1)}, \varepsilon^{(1)^{2}}=-1, \varepsilon^{(1)} \cdot \varepsilon^{(3)}=\varepsilon^{(1)} \cdot n=0$, and defines the second transverse polarization vector

$$
\varepsilon^{(2)^{\mu}}=\varepsilon^{\alpha \beta \mu \gamma} n_{\alpha} \varepsilon_{\beta}^{(1)} \varepsilon_{\gamma}^{(3)}
$$


The spacelike parts of $\varepsilon^{(1)}, \varepsilon^{(2)}$, and $\varepsilon^{(3)}$ build a right-handed coordinate system. The polarization vectors for a massless spin- 1 particle with spin projection $+1,0$, and -1 are then $[17]$

$$
\begin{aligned}
\varepsilon^{+} & =\frac{1}{\sqrt{2}}\left(\varepsilon^{(1)}+i \varepsilon^{(2)}\right), \\
\varepsilon^{0} & =\varepsilon^{(3)}, \\
\varepsilon^{-} & =\frac{1}{\sqrt{2}}\left(\varepsilon^{(1)}-i \varepsilon^{(2)}\right) .
\end{aligned}
$$

According to eq. 1.9, the desired wave functions are

$$
\psi_{\mu}^{ \pm}(P)=\varepsilon_{\mu}^{ \pm} u^{ \pm}(P)
$$

Obviously, $\psi_{\mu}^{ \pm}$satisfy the second Rarita-Schwinger equation 1.3. In order to see that eq. 1.2 is satisfied as well, note that $\ddagger^{(2)}=-i \gamma_{5} h \phi^{(1)} \ddagger^{(3)}$ (using the identity eq. 1.7), and that $\notin^{(3)} u^{ \pm}=-\not h u^{ \pm}$. With this, one finds indeed

$$
\gamma^{\mu} \psi_{\mu}^{ \pm}=\frac{1}{\sqrt{2}} \oiint^{(1)}\left(1 \mp \gamma_{5}\right) u^{ \pm}=0 .
$$

Unlike eq. 1.12, the massless wave functions are normalized to

$$
\bar{\psi}_{\mu}^{l} \gamma^{\rho} \psi^{l^{\prime} \mu}=-2 P^{\rho} \delta^{l l^{\prime}}
$$

The projection operator $\Pi_{\mu \nu}^{\frac{3}{2}}(P)$ for massless spin-3/2 particles is

$$
\begin{aligned}
\Pi_{\mu \nu}^{\frac{3}{2}}(P) & =\sum_{l=+3 / 2,-3 / 2} \psi_{\mu}^{l}(P) \bar{\psi}_{\nu}^{l}(P)=\left[\varepsilon_{\mu}^{+} \varepsilon_{\nu}^{+*}\left(1+\gamma_{5}\right)+\varepsilon_{\mu}^{-} \varepsilon_{\nu}^{-*}\left(1-\gamma_{5}\right)\right] \frac{\not P}{2} \\
& =\frac{\not P}{2}\left[\varepsilon_{\mu}^{(1)} \varepsilon_{\nu}^{(1)}+\varepsilon_{\mu}^{(2)} \varepsilon_{\nu}^{(2)}+i \gamma_{5}\left(\varepsilon_{\mu}^{(1)} \varepsilon_{\nu}^{(2)}-\varepsilon_{\mu}^{(2)} \varepsilon_{\nu}^{(1)}\right)\right] .
\end{aligned}
$$

$\Pi_{\mu \nu}^{\frac{3}{2}}(P)$ can be shown to satisfy the Rarita-Schwinger equations 1.14 (with $m=0$ ) and to possess the projector property (eq. 1.15 modified to the massless case)

$$
\Pi_{\mu \lambda}^{\frac{3}{2}}(P) \gamma_{\rho} \Pi_{\nu}^{\frac{3}{2} \lambda}(P)=-2 P_{\rho} \Pi_{\mu \nu}^{\frac{3}{2}}(P),
$$

just as $\Pi_{\mu \nu}(P)$ does.

The use of this projection operator in a practical calculation leads to scalar products and pseudoscalars involving the vectors $\varepsilon^{(1)}$ and $\varepsilon^{(2)}$, for which we do not have covariant expressions. However, we are free to use a particularly convenient coordinate system to calculate those quantities, for example the coordinate system in which $P$ has components

$$
P^{\mu}=(p, 0,0, p) .
$$


Choosing the time axis

$$
n^{\mu}=(1,0,0,0)
$$

one finds

$$
\varepsilon^{(3)^{\mu}}=(0,0,0,1),
$$

and with the choice

$$
\varepsilon^{(1)^{\mu}}=(0,1,0,0)
$$

$\varepsilon^{(2)}$ becomes

$$
\varepsilon^{(2)^{\mu}}=(0,0,1,0) .
$$

Here one sees explicitly that $P \cdot \varepsilon^{(1)}=P \cdot \varepsilon^{(2)}=0$. If the calculation in question is an inelastic $2 \rightarrow 2$ process

$$
a\left(P_{1}\right)+b\left(P_{2}\right) \rightarrow c\left(P_{3}\right)+d\left(P_{4}\right)
$$

where a spin-3/2 particle $d$ is produced, one can go to the CMS system, where

$$
\begin{aligned}
& P_{2}^{\mu}=(p, 0, p \sin \alpha, p \cos \alpha), \\
& P_{3}^{\mu}=(p, 0,0,-p) .
\end{aligned}
$$

Here all particle masses have been neglected (the formalism can easily be generalized to massive particles $a, b$, and $c$ ). $\alpha$ is the CMS scattering angle and $p$ the CMS energy; $\cos \alpha$ and $p$ can be expressed in terms of the Mandelstam variables $s$ and $t$. The representation eq. 1.35 will be used in section 2.1 to calculate an inelastic $2 \rightarrow 2$ gravitino production cross section. The quantities required there are the scalar products

$$
\begin{aligned}
P_{3} \cdot \varepsilon^{(1)}=P_{3} \cdot \varepsilon^{(2)}= & P_{2} \cdot \varepsilon^{(1)}=0, \\
& P_{2} \cdot \varepsilon^{(2)}=-p \sin \alpha,
\end{aligned}
$$

and the pseudoscalars (with $\varepsilon^{0123}=-\varepsilon_{0123}=+1$ )

$$
\begin{aligned}
& \varepsilon^{\mu \nu \rho \sigma} P_{2 \mu} P_{3 \nu} P_{4 \rho} \varepsilon_{\sigma}^{(1)}=2 p^{3} \sin \alpha, \\
& \varepsilon^{\mu \nu \rho \sigma} P_{2 \mu} P_{3 \nu} \varepsilon_{\rho}^{(1)} \varepsilon_{\sigma}^{(2)}=p^{2}(1+\cos \alpha), \\
& \varepsilon^{\mu \nu \rho \sigma} P_{2 \mu} P_{4 \nu} \varepsilon_{\rho}^{(1)} \varepsilon_{\sigma}^{(2)}=p^{2}(\cos \alpha-1), \\
& \varepsilon^{\mu \nu \rho \sigma} P_{3 \mu} P_{4 \nu} \varepsilon_{\rho}^{(1)} \varepsilon_{\sigma}^{(2)}=-2 p^{2} .
\end{aligned}
$$




\subsection{Effective theory for light gravitinos}

If in an ordinary gauge theory a global gauge symmetry is broken, Goldstone bosons are created. In the case of a local rather than global symmetry, according to the Higgs mechanism the Goldstone bosons are eaten by the gauge bosons, giving them mass. If a globally supersymmetric theory is spontaneously broken, a massless spin-1/2 Majorana particle is created, the goldstino. In a locally supersymmetric theory, i.e. in supergravity, the goldstino degrees of freedom are eaten by the gravitino through the super-Higgs mechanism [2]. Thus the gravitino acquires mass and two additional degrees of freedom, the helicity $\pm \frac{1}{2}$ components.

This suggests that the interactions of the helicity $\pm \frac{1}{2}$ components of the gravitino can be approximated by goldstino dynamics. Indeed, in the limit of vanishing gravitino mass $m_{\tilde{G}}$ and vanishing gravitational coupling $\frac{1}{M}$, the helicity $\pm \frac{3}{2}$ components of the gravitino are negligible and its helicity $\pm \frac{1}{2}$ components behave exactly as the goldstino of spontaneously broken globally supersymmetric theories, with goldstino-matter rather than gravitational interaction strength [3].

The gravitino-goldstino high-energy equivalence theorem [21] states that "the S-matrix elements for longitudinally polarized gravitinos (helicities $\pm \frac{1}{2}$ ) and other physical particles are asymptotically equal up to corrections of the order $m_{\tilde{G}} / \sqrt{s}$ to corresponding S-matrix elements where each longitudinally polarized gravitino is replaced by the corresponding goldstino." So at large momenta the helicity $\pm \frac{1}{2}$ components of the gravitino are essentially given by a goldstino, and the gravitino wave function is

$$
\psi_{\mu}^{ \pm \frac{1}{2}}(P)=i \sqrt{\frac{2}{3}} \frac{P_{\mu}}{m_{\tilde{G}}} u^{ \pm}(P)+\mathcal{O}\left(\frac{m_{\tilde{G}}}{|\mathbf{p}|}\right)
$$

Since for light gravitinos, the interactions of the helicity $\pm \frac{1}{2}$ components are much stronger than the interactions of the $\pm \frac{3}{2}$ components, it is possible to build an effective theory for light gravitinos in which only the helicity $\pm \frac{1}{2}$ components are left over. The effective theory has the advantage to avoid the complicated spin- $\frac{3}{2}$ formalism in calculations.

There are two ways to get the Feynman rules for light gravitinos in the effective theory: Either one replaces in the original supergravity Lagrangian the gravitino wave function according to eq. 1.38 and uses the equations of motion of the particles to get rid of the derivatives by integration by parts, or one uses goldstino interaction vertices from a Lagrangian in which global supersymmetry has been broken.

For the calculations presented here, the Feynman rules for light gravitinos have been derived from the effective Lagrangian of [22]. Compared to the Feynman rules for light 
gravitinos used in [11] this Lagrangian contains a new quartic term leading to a gravitinogluino-squark-squark vertex, which is not present in the full theory. As pointed out in [22], including the Feynman diagram with this new vertex leads to a cross section for gravitino production process $\mathrm{J}$ (see section 2.2 ) which is four times as large as the result found by [11] for the same cross section without the additional diagram. The larger result is consistent with the result from the full theory which is the sum of the results for small and large gravitino masses (see section 2.2). The effective Lagrangian and the Feynman rules of the effective theory for light gravitinos are given in appendix A. 


\section{Chapter 2}

\section{Gravitino Production Processes}

In this chapter the Boltzmann collision term which describes the rate of change of the gravitino number density is derived from considering the ten dominant inelastic $2 \rightarrow 2$ gravitino production processes. First, the calculation of the differential cross section for one of the processes is given in detail. Then the results for the other nine processes are summarized. For four of the ten processes the total cross section diverges due to the exchange of massless infrared gluons, so one has to choose a regularization scheme. Two possible schemes are presented, the ad hoc introduction of a thermal gluon mass and an angular cut off. Both schemes lead to a logarithmic term in the total cross section with the same coefficient for the logarithm, but different constant terms. The Boltzmann collision term is then obtained by integrating the individual squared matrix elements over phase space. Usually this is done neglecting the quantum statistical factors in the final phase space. In appendix $\mathrm{B}$ a method is explained of how to carry out all phase space integrations in the lab system, i. e. in the rest system of the thermal bath. This allows to take into account those usually neglected quantum corrections. The coefficient of the leading logarithmic term in the Boltzmann collision term can be calculated analytically, and it will turn out that with quantum corrections, it is the same as the one obtained from the calculation of the gravitino self energy in chapter 3 .

\subsection{Example of a $2 \rightarrow 2$ cross section for gravitino pro- duction}

Here the calculation of the differential cross section of the process gluon + gluon $\rightarrow$ gluino + gravitino is demonstrated in detail. I have chosen this particular process because it is an example for a process where contributions from unphysical gluon polarizations must be 
subtracted somehow. Usually this is done by subtracting a ghost diagram. An alternative way is to use an expression for the sum over the gluon polarisations in which the unphysical contributions are already subtracted [23]. Both methods are demonstrated here. As it should be, they lead to the same result.

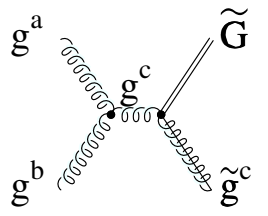

(a)

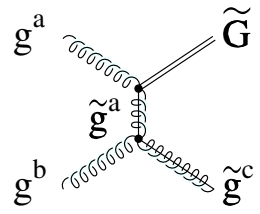

(b)

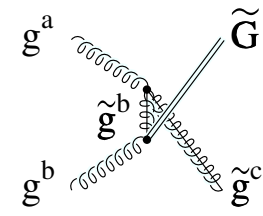

(c)

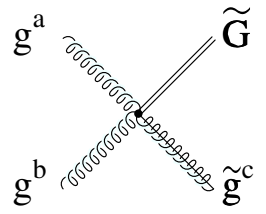

(d)

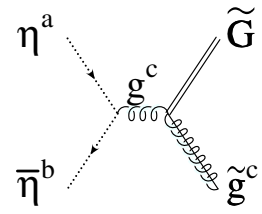

(e)

Figure 2.1: Feynman diagrams of process gluon + gluon $\rightarrow$ gluino + gravitino

On the tree level, for the process under consideration one has the four Feynman diagrams (a) to (d) in fig. 2.1 and, if one chooses to work with ghosts, the ghost diagram (e), which is the $s$-channel diagram (a) with the external gluons replaced by ghosts.

The particles have four-momenta $P_{1}$ to $P_{4}$, and the gluons/ghosts and gluinos have colour indices $a, b$, and $c$, so the process reads

$$
g^{a}\left(P_{1}\right)+g^{b}\left(P_{2}\right) \rightarrow \tilde{g}^{c}\left(P_{3}\right)+\tilde{G}\left(P_{4}\right) .
$$

Let us calculate the squared matrix element resulting from the four diagrams (a) to (d). With $\varepsilon_{a}^{\mu}, \varepsilon_{b}^{\nu}$ denoting the polarization vectors of the two gluons, the expression to be evaluated reads

$$
\varepsilon_{a}^{\mu} \varepsilon_{a}^{* \mu^{\prime}} \varepsilon_{b}^{\nu} \varepsilon_{b}^{* \nu^{\prime}} D_{\mu \mu^{\prime} \nu \nu^{\prime}}=\varepsilon_{a}^{\mu} \varepsilon_{a}^{* \mu^{\prime}} \varepsilon_{b}^{\nu} \varepsilon_{b}^{* \nu^{\prime}} \operatorname{tr}\left[\bar{\psi}^{\rho}\left(P_{4}\right) V_{\rho \mu \nu}\left(P_{1}, P_{2}, P_{3}, P_{4}\right) v\left(P_{3}\right) \bar{v} \bar{V}_{\rho^{\prime} \mu^{\prime} \nu^{\prime}} \psi^{\rho^{\prime}}\right]
$$

where $D_{\mu \mu^{\prime} \nu \nu^{\prime}}$ is a Dirac trace with propagator denominators, and $V_{\rho \mu \nu}\left(P_{1}, P_{2}, P_{3}, P_{4}\right)$ is the sum of the four vertex in diagram $(d)$ and the three products of three vertices and propagators from the $s^{-}, t^{-}$, and $u$-channel diagram (a), (b), and (c). Here and later I always sum over initial and final spin states.

Using the Feynman rules from appendix $A$ and taking the trace with FORM one gets an expression for $D_{\mu \mu^{\prime} \nu \nu^{\prime}}$ in terms of the momenta $P_{2}, P_{3}$, and $P_{4}$ ( $P_{1}$ is eliminated using energy-momentum conservation) and of the masses $m_{\tilde{G}}$ and $m_{\tilde{g}}$. Note that $D_{\mu \mu^{\prime} \nu \nu^{\prime}}$ is gauge independent: the part of the gluon propagator which depends on the gauge parameter $\xi$ is proportional to the gluon momentum. In the gravitino-gluon-gluino vertex this gluon momentum is contracted with a commutator that contains another gluon momentum, yielding zero. 
If the calculation is done with ghosts, one replaces

$$
\sum_{\text {spins }} \varepsilon_{a}^{\mu} \varepsilon_{a}^{* \mu^{\prime}} \rightarrow-g^{\mu \mu^{\prime}}, \sum_{\text {spins }} \varepsilon_{b}^{\nu} \varepsilon_{b}^{* \nu^{\prime}} \rightarrow-g^{\nu \nu^{\prime}}
$$

With these replacements in eq. 2.2, let us call our expression $\left|\mathcal{M}_{4}\right|^{2}$. Introducing the Mandelstam variables

$$
\begin{aligned}
s & =\left(P_{1}+P_{2}\right)^{2}, \\
t & =\left(P_{1}-P_{3}\right)^{2}, \\
u & =\left(P_{1}-P_{4}\right)^{2} \\
& =-s-t+m_{\tilde{g}}^{2}+m_{\tilde{G}}^{2},
\end{aligned}
$$

one gets replacements for the scalar products $P_{2} \cdot P_{3}, P_{2} \cdot P_{4}$, and $P_{3} \cdot P_{4}$, and ends up with an expression for $\left|\mathcal{M}_{4}\right|^{2}$ in terms of $s, t, m_{\tilde{G}}$, and $m_{\tilde{g}}$.

So far the calculation was exact. Now we want to take the high energy limit $s \gg m_{\tilde{g}}, m_{\tilde{G}}$. In order to keep track of the different orders in the expansion, we multiply $\left|\mathcal{M}_{4}\right|^{2}$ by the denominators of the propagators of massive particles, i.e. by $\left(t-m_{\tilde{g}}\right)^{2}\left(u-m_{\tilde{g}}^{2}\right)$.

There are terms in the resulting expression containing no mass factors, so all similar terms with mass factors instead of factors $s$ or $t$ can be neglected.

However, care has to be taken with terms that have a denominator $\frac{1}{m_{\tilde{G}}^{2}}$ arising from the gravitino spin sum eq. 1.16. It turns out that in the differential cross sections of all ten $2 \rightarrow 2$-gravitino production processes such denominators are balanced by a product of at least two masses $m_{i}$, where $m_{i}$ stands for the mass of any of the other particles involved, i.e. gluinos, quarks, or squarks. These terms are dealt with assuming that

$$
\frac{m_{i}^{2}}{m_{\tilde{G}}^{2}} \ll \frac{s}{m_{i}^{2}} .
$$

(Factors $t$ have to be treated in the same way as factors $s$, since we are interested in the leading terms of the total cross section, which is up to a factor the integral of the differential cross section over $t$ from $-s$ to 0.$)\left|\mathcal{M}_{4}\right|^{2}$ however contains also terms $\propto \frac{1}{m_{\tilde{G}}^{2}}$ with no other masses in the numerator. These terms will be canceled by like terms from the ghost diagram. In order to get the high energy limit of $\left|\mathcal{M}_{4}\right|^{2}$ one therefore has to keep three classes of terms: terms with no mass factors, terms with mass factors $\frac{m_{\tilde{g}}^{2}}{m_{\tilde{G}}^{2}}$, and terms $\propto \frac{1}{m_{\tilde{\tilde{\sigma}}}^{2}}$ with no other masses in the numerator. We divide again by $t^{2}(s+t)^{2}$, thus realizing the limit $s \gg m_{\tilde{g}}, m_{\tilde{G}}$ also for the propagator denominators. Note that by merely replacing $\frac{1}{t-m_{\tilde{g}}^{2}}$ by $\frac{1}{t}$, one could have lost terms of the order of $\frac{m_{\tilde{g}}^{2}}{m_{\tilde{G}}^{2}}$. 
The result is

$$
\left|\mathcal{M}_{4}\right|^{2}=\frac{2}{3} \frac{g^{2}\left|f^{a b c}\right|^{2}}{M^{2}}\left(\frac{7 s^{2}+18 s t+15 t^{2}}{s}+\frac{m_{\tilde{g}}^{2}}{m_{\tilde{G}}^{2}} \frac{4 s^{2}+8 s t+5 t^{2}}{s}-\frac{t(s+t)}{m_{\tilde{G}}^{2}}\right) .
$$

The replacement eq. 2.3 introduces contributions from the unphysical longitudinal polarization of the gluons. These contributions are exactly given by twice the ghost diagram (e), which we will subtract from $\left|\mathcal{M}_{4}\right|^{2}$. The calculation of the modulus squared of the ghost diagram $\left|\mathcal{M}_{\eta}\right|^{2}$ follows the same lines as the calculation of $\left|\mathcal{M}_{4}\right|^{2}$, and one obtains

$$
\left|\mathcal{M}_{\eta}\right|^{2}=\frac{1}{3} \frac{g^{2}\left|f^{a b c}\right|^{2}}{M^{2}}\left(\frac{s^{2}+6 s t+3 t^{2}}{s}+\frac{m_{\tilde{g}}^{2}}{m_{\tilde{G}}^{2}} \frac{2 s^{2}+4 s t+t^{2}}{s}-\frac{t(s+t)}{m_{\tilde{G}}^{2}}\right) .
$$

Ghost lines carry a direction arrow corresponding to ghost and antighost. One has to take into account the contributions from both directions, therefore $\left|\mathcal{M}_{\eta}\right|^{2}$ is multiplied by a factor of two, and the final result reads

$$
\begin{aligned}
\left|\mathcal{M}_{A}\right|^{2} & =\left|\mathcal{M}_{4}\right|^{2}-2\left|\mathcal{M}_{\eta}\right|^{2} \\
& =4 \frac{g^{2}\left|f^{a b c}\right|^{2}}{M^{2}}\left(1+\frac{m_{\tilde{g}}^{2}}{3 m_{\tilde{G}}^{2}}\right)\left(s+2 t+2 \frac{t^{2}}{s}\right) .
\end{aligned}
$$

An alternative way to get rid of the contributions from longitudinal gluons and at the same time a check of the calculation is to use the correct spin sum [23]. If one takes instead of the replacement eq. 2.3 the spin sums

$$
\begin{aligned}
& \sum_{\text {spins }} \varepsilon_{a}^{\mu} \varepsilon_{a}^{* \mu^{\prime}}=-g^{\mu \mu^{\prime}}+\frac{2}{s}\left(P_{1}^{\mu} P_{2}^{\mu^{\prime}}+P_{1}^{\mu^{\prime}} P_{2}^{\mu}\right), \\
& \sum_{\text {spins }} \varepsilon_{b}^{\nu} \varepsilon_{b}^{* \nu^{\prime}}=-g^{\nu \nu^{\prime}}+\frac{2}{s}\left(P_{1}^{\nu} P_{2}^{\nu^{\prime}}+P_{1}^{\nu^{\prime}} P_{2}^{\nu}\right),
\end{aligned}
$$

then one can obtain the result eq. 2.8 directly from

$$
\left|\mathcal{M}_{A}\right|^{2}=\varepsilon_{a}^{\mu} \varepsilon_{a}^{* \mu^{\prime}} \varepsilon_{b}^{\nu} \varepsilon_{b}^{* \nu^{\prime}} D_{\mu \mu^{\prime} \nu \nu^{\prime}}
$$

So far we have calculated the modulus squared of our matrix element using the full gravitino wave function, i.e. summing over all four helicity states. The result is proportional to the characteristic factor $\left(1+\frac{m_{\tilde{g}}^{2}}{3 m_{\tilde{G}}^{2}}\right)$ which will appear also in all the other $2 \rightarrow 2$-gravitino production processes. Interestingly, this result is just the sum of of the two limiting cases of very big or very small gravitino mass; there is nothing new from the gravitino mass range $m_{\tilde{G}} \approx m_{\tilde{g}}$.

For very heavy gravitinos, only the gravitino components with maximal helicity play a role. Their contribution to $\left|\mathcal{M}_{A}\right|^{2}$ can be obtained with the formalism presented in 
section 1.3. For the sum over gravitino helicities $\sum_{\text {spins }} \psi^{\rho^{\prime}} \bar{\psi}^{\rho}$ in the Dirac trace eq. 2.2 one simply has to use $\Pi^{\frac{3}{2} \rho^{\prime} \rho}$ instead of $\Pi^{\rho^{\prime} \rho}$. The scalar and pseudo scalar products which need to be evaluated are given in eqs. 1.36 and 1.37. With the definitions eq. 2.4 and eq. 1.35, one has for the CMS energy and scattering angle

$$
\begin{aligned}
p & =\frac{\sqrt{s}}{2}, \\
\cos \alpha & =2 \frac{t}{s}+1 .
\end{aligned}
$$

The result for the squared matrix element is

$$
\left|\mathcal{M}_{A}^{\frac{3}{2}}\right|^{2}=4 \frac{g^{2}\left|f^{a b c}\right|^{2}}{M^{2}}\left(s+2 t+2 \frac{t^{2}}{s}\right) .
$$

For very light gravitinos, the effective theory of section 1.4 can be used. With the vertices given in the appendix A.3.3 and the spin sum $\sum_{\text {spins }} \psi \bar{\psi}=\not P_{4}$ in the Dirac trace eq. 2.2 one gets

$$
\left|\mathcal{M}_{A}^{\mathrm{eff}}\right|^{2}=\frac{4}{3} \frac{g^{2}\left|f^{a b c}\right|^{2} m_{\tilde{g}}^{2}}{M^{2} m_{\tilde{G}}^{2}}\left(s+2 t+2 \frac{t^{2}}{s}\right)
$$

\subsection{Summary of all ten $2 \rightarrow 2$ cross sections}

In total, there are ten different inelastic $2 \rightarrow 2$ scattering processes in which gravitinos can be produced. Following [10] I denote them by the letters A to J; the processes along with the contributing Feynman diagrams at tree level are listed below:

- $\mathrm{A}: g^{a}+g^{b} \rightarrow \tilde{g}^{c}+\tilde{G}$

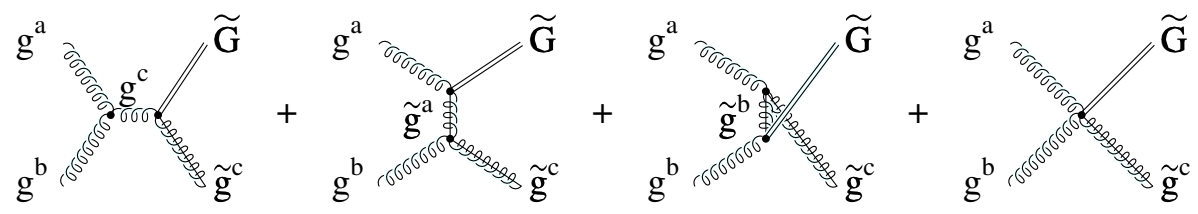

- B: $g^{a}+\tilde{g}^{b} \rightarrow g^{c}+\tilde{G}(\operatorname{crossing}$ of $\mathrm{A})$

- $\mathrm{C}: \tilde{q}_{i}+g^{a} \rightarrow \tilde{q}_{j}+\tilde{G}$

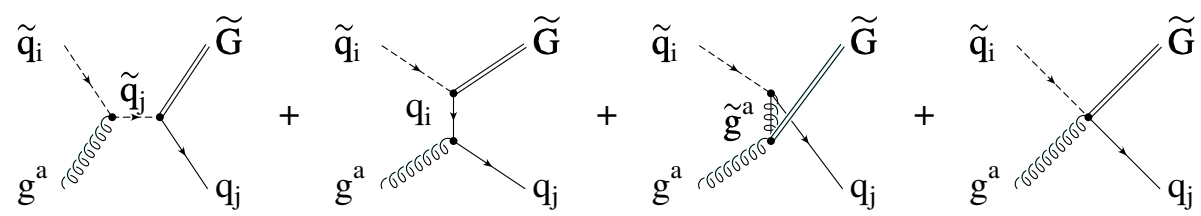


- D: $q_{i}+g^{a} \rightarrow \tilde{q}_{j}+\tilde{G}($ crossing of $\mathrm{C})$

- E: $q_{i}+\overline{\tilde{q}}_{j} \rightarrow g^{a}+\tilde{G}(\operatorname{crossing}$ of $\mathrm{C})$

- $\mathrm{F}: \tilde{g}^{a}+\tilde{g}^{b} \rightarrow \tilde{g}^{c}+\tilde{G}$

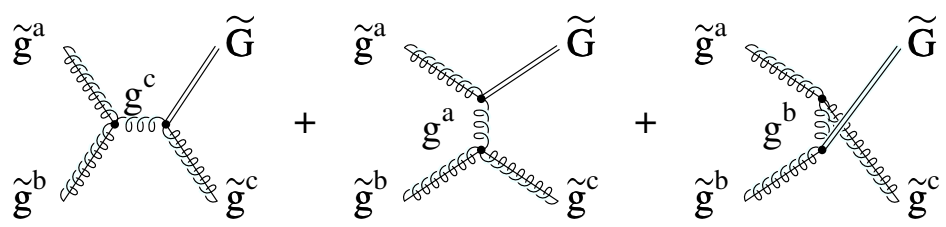

- $\mathrm{G}: q_{i}+\tilde{g}^{a} \rightarrow q_{j}+\tilde{G}$

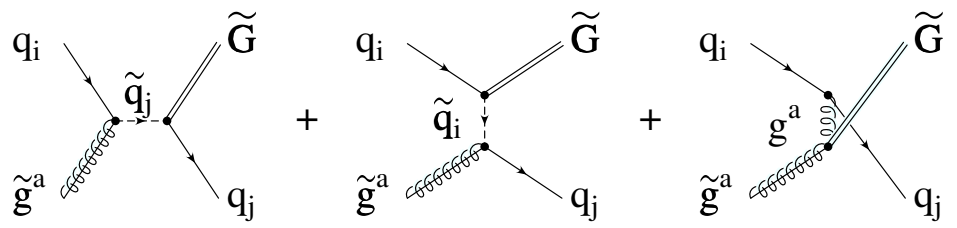

- $\mathrm{H}: \tilde{q}_{i}+\tilde{g}^{a} \rightarrow \tilde{q}_{j}+\tilde{G}$

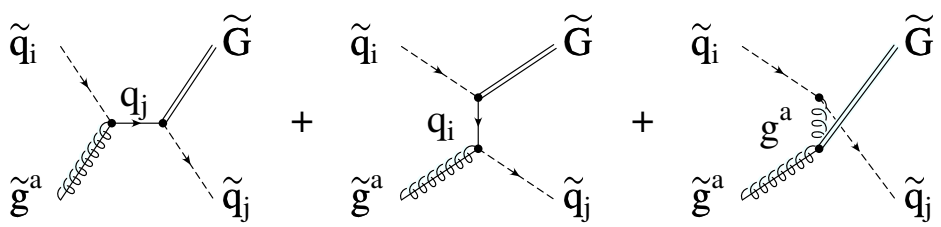

- I: $q_{i}+\bar{q}_{j} \rightarrow \tilde{g}^{a}+\tilde{G}($ crossing of $\mathrm{G})$

- J: $\tilde{q}_{i}+\overline{\tilde{q}}_{j} \rightarrow \tilde{g}^{a}+\tilde{G}($ crossing of $\mathrm{H})$

Note that in the effective theory for light gravitinos the fourth diagram with the gravitinoquark-squark-gluon vertex in processes $\mathrm{C}, \mathrm{D}$, and $\mathrm{E}$ is missing. Instead there is a fourth diagram with the gravitino-gluino-squark-squark vertex in processes $\mathrm{H}$ and $\mathrm{J}$ in the effective theory.

In the previous section it has been shown in detail how the high energy limit of the squared matrix element of process $\mathrm{A},\left|\mathcal{M}_{A}\right|^{2}$, is obtained. It turned out to be the sum of the two limiting cases of very big and very small gravitino mass. In the same way the high energy limit of the squared matrix elements of the other nine processes are calculated, and again they are simply the sum of the two limiting cases, with no new contribution from an intermediate gravitino mass range. 


\begin{tabular}{l|c|l} 
& process $i$ & $\left|\mathcal{M}_{i}\right|^{2} / \frac{g^{2}}{M^{2}}\left(1+\frac{m_{\tilde{g}}^{2}}{3 m_{\tilde{\sigma}}^{2}}\right)$ \\
\hline \hline $\mathrm{A}$ & $g^{a}+g^{b} \rightarrow \tilde{g}^{c}+\tilde{G}$ & $4\left(s+2 t+2 \frac{t^{2}}{s}\right)\left|f^{a b c}\right|^{2}$ \\
\hline $\mathrm{B}$ & $g^{a}+\tilde{g}^{b} \rightarrow g^{c}+\tilde{G}$ & $-4\left(t+2 s+2 \frac{s^{2}}{t}\right)\left|f^{a b c}\right|^{2}$ \\
\hline $\mathrm{C}$ & $g^{a}+\tilde{q}_{i} \rightarrow q_{j}+\tilde{G}$ & $2 s\left|T_{j i}^{a}\right|^{2}$ \\
\hline $\mathrm{D}$ & $g^{a}+q_{i} \rightarrow \tilde{q}_{j}+\tilde{G}$ & $2(s+t)\left|T_{j i}^{a}\right|^{2}$ \\
\hline $\mathrm{E}$ & $q_{i}+\overline{\tilde{q}}_{j} \rightarrow g^{a}+\tilde{G}$ & $2(s+t)\left|T_{j i}^{a}\right|^{2}$ \\
\hline $\mathrm{F}$ & $\tilde{g}^{a}+\tilde{g}^{b} \rightarrow \tilde{g}^{c}+\tilde{G}$ & $-8 \frac{\left(s^{2}+s t+t^{2}\right)^{2}}{s t(s+t)}\left|f^{a b c}\right|^{2}$ \\
\hline $\mathrm{G}$ & $\tilde{g}^{a}+q_{i} \rightarrow q_{j}+\tilde{G}$ & $-4\left(s+\frac{s^{2}}{t}\right)\left|T_{j i}^{a}\right|^{2}$ \\
\hline $\mathrm{H}$ & $\tilde{g}^{a}+\tilde{q}_{i} \rightarrow \tilde{q}_{j}+\tilde{G}$ & $-2\left(t+2 s+2 \frac{s^{2}}{t}\right)\left|T_{j i}^{a}\right|^{2}$ \\
\hline $\mathrm{I}$ & $q_{i}+\bar{q}_{j} \rightarrow \tilde{g}^{a}+\tilde{G}$ & $-4\left(t+\frac{t^{2}}{s}\right)\left|T_{j i}^{a}\right|^{2}$ \\
\hline $\mathrm{J}$ & $\tilde{q}_{i}+\overline{\tilde{q}}_{j} \rightarrow \tilde{g}^{a}+\tilde{G}$ & $2\left(s+2 t+2 \frac{t^{2}}{s}\right)\left|T_{j i}^{a}\right|^{2}$
\end{tabular}

Table 2.1: Squared matrix elements $\left|\mathcal{M}_{i}\right|^{2}$ up to a factor of $\frac{g^{2}}{M^{2}}\left(1+\frac{m_{\tilde{g}}^{2}}{3 m_{\tilde{G}}^{2}}\right)$ for gravitino $(\tilde{G})$ production in two-body processes involving left-handed quarks $\left(q_{i}\right)$, scalar quarks $\left(\tilde{q}_{i}\right)$, gluons $\left(g^{a}\right)$ and gluinos $\left(\tilde{g}^{a}\right)$. The values are given for the specified choice of colors and summed over spins in the initial and final state. $f^{a b c}$ and $T_{j i}^{a}$ are the usual SU(3) colour matrices.

In table 2.1 the squared matrix elements of all ten processes are shown. The values given are summed over initial and final spin states. As far as chiral fermions and their scalar partners are involved, only contributions for left-handed particles are included.

A nice check of the $\left|\mathcal{M}_{i}\right|^{2}$ is provided by their crossing symmetries. The processes $\mathrm{A}$ and $\mathrm{B}, \mathrm{G}$ and $\mathrm{I}$, and $\mathrm{H}$ and $\mathrm{J}$ represent pairs of $s$ - and $t$-channel processes. One gets $\left|\mathcal{M}_{B}\right|^{2}$ from $\left|\mathcal{M}_{A}\right|^{2}$ by exchanging every $s$ for a $t$ and vice versa, and by multiplying by a factor of -1 for each fermion crossed from the initial to the final state or in the other sense. The two other pairs of processes behave the same way.

The processes $\mathrm{C}, \mathrm{D}$, and $\mathrm{E}$ are three crossing channels of the same reaction. Consider first the processes $\mathrm{C}$ and $\mathrm{D}$. If we try to view them as $s$ - and $t$-channel, starting from $\left|\mathcal{M}_{C}\right|^{2} \propto s$, we would expect $\left|\mathcal{M}_{D}\right|^{2} \propto t$ rather than $\left|\mathcal{M}_{D}\right|^{2} \propto(s+t)$. So we should interpret $\mathrm{D}$ as the $u$-channel reaction, but the same is true for process $\mathrm{E}$. Where is the $t$ - 
channel? The answer is that $t$ and $u$ cannot be defined unambiguously for three different processes, because one can measure the scattering angle of an outgoing particle with respect to both ingoing particles, and none is preferred. Switching from one to the other corresponds to exchanging a factor $-t$ for a factor $s-t$ and vice versa (in the case of the process pairs $\mathrm{A}$ and $\mathrm{B}, \mathrm{G}$ and $\mathrm{I}$, and $\mathrm{H}$ and $\mathrm{J}$ things are different, because for each pair the third channel would not represent a new process). Of course, the total cross section, which is obtained by integrating over $t$, is not affected by this choice.

Another consequence of crossing symmetry is the particular form of $\left|\mathcal{M}_{F}\right|^{2}$. Process $\mathrm{F}$ is the process with three gluinos. All three crossing channels look the same, therefore $\left|\mathcal{M}_{F}\right|^{2}$ is invariant under the exchange $s \leftrightarrow t$ or $s \leftrightarrow u$.

\subsection{Two cut-off methods}

In this section the total cross sections $\sigma_{i}$ for the ten processes $\mathrm{A}$ to $\mathrm{J}$ are calculated. The $\sigma_{i}$ are related to the squared matrix elements $\left|\mathcal{M}_{i}\right|^{2}$ by

$$
\sigma_{i}=\int \frac{d^{3} p_{3}}{(2 \pi)^{3} 2 E_{3}} \frac{d^{3} p_{4}}{(2 \pi)^{3} 2 E_{4}}(2 \pi)^{4} \delta\left(P_{1}+P_{2}-P_{3}-P_{4}\right) \frac{\left|\mathcal{M}_{i}\right|^{2}}{4 I}
$$

Here, the momenta are as in process A, eq. 2.1. $P_{i}^{\mu}=\left(E_{i}, \mathbf{p}_{i}\right)$, and $I$ is the flux factor, $I=\frac{1}{2} w\left(s, m_{1}^{2}, m_{2}^{2}\right)$ with the function $w(x, y, z)$ defined by

$$
w(x, y, z)=\sqrt{x^{2}+y^{2}+z^{2}-2(x y+x z+y z)} .
$$

This definition of $\sigma$ differs from the usual definition of a total cross section in that I have summed over spins in both initial and final state. Usually the delta function in eq. 2.14 is integrated out in the CMS system (see for example [24]), and one is left with an integral over the differential cross section:

$$
\sigma_{i}=\int d t \frac{d \sigma_{i}}{d t}=\int_{t_{-}}^{t_{+}} d t \frac{\left|\mathcal{M}_{i}\right|^{2}}{64 \pi I^{2}}
$$

The exact integration limits are functions of $s$ and the masses of the four particles involved in the process:

$$
t_{ \pm}=m_{1}^{2}+m_{3}^{2}-\frac{1}{2 s}\left[\left(s+m_{1}^{2}-m_{2}^{2}\right)\left(s+m_{3}^{2}-m_{4}^{2}\right) \pm w\left(s, m_{1}^{2}, m_{2}^{2}\right) w\left(s, m_{3}^{2}, m_{4}^{2}\right)\right] .
$$

In the high energy limit $s \gg m_{i}^{2}$ one has $w\left(s, m_{i}^{2}, m_{j}^{2}\right) \approx s$, and eq. 2.16 simplifies considerably:

$$
\sigma_{i}=\int d t \frac{d \sigma_{i}}{d t}=\int_{-s}^{0} d t \frac{\left|\mathcal{M}_{i}\right|^{2}}{16 \pi s^{2}}
$$




\begin{tabular}{c|c|c} 
& process $i$ & $\sigma_{i} / F$ \\
\hline \hline $\mathrm{A}$ & $g^{a}+g^{b} \rightarrow \tilde{g}^{c}+\tilde{G}$ & $\frac{8}{3}\left|f^{a b c}\right|^{2}$ \\
\hline $\mathrm{C}$ & $g^{a}+\tilde{q}_{i} \rightarrow q_{j}+\tilde{G}$ & $2\left|T_{j i}^{a}\right|^{2}$ \\
\hline $\mathrm{D}$ & $g^{a}+q_{i} \rightarrow \tilde{q}_{j}+\tilde{G}$ & $\left|T_{j i}^{a}\right|^{2}$ \\
\hline $\mathrm{E}$ & $q_{i}+\overline{\tilde{q}}_{j} \rightarrow g^{a}+\tilde{G}$ & $\left|T_{j i}^{a}\right|^{2}$ \\
\hline $\mathrm{I}$ & $q_{i}+\bar{q}_{j} \rightarrow \tilde{g}^{a}+\tilde{G}$ & $\frac{2}{3}\left|T_{j i}^{a}\right|^{2}$ \\
\hline $\mathrm{J}$ & $\tilde{q}_{i}+\overline{\tilde{q}}_{j} \rightarrow \tilde{g}^{a}+\tilde{G}$ & $\frac{4}{3}\left|T_{j i}^{a}\right|^{2}$
\end{tabular}

Table 2.2: Total cross sections $\sigma_{i}$ up to a factor of $F=\frac{g^{2}}{16 \pi M^{2}}\left(1+\frac{m_{\tilde{g}}^{2}}{3 m_{\tilde{G}}^{2}}\right)$ for gravitino production; processes with no divergence. The values are given for the specified choice of colors and summed over spins in the initial and final state. As for chiral particles, only one chirality is included.

With this it is easy to obtain the total cross sections of the processes A, C, D, E, I, and J. They are given in table 2.2 .

The remaining processes $\mathrm{B}, \mathrm{F}, \mathrm{G}$, and $\mathrm{H}$ however give logarithmically divergent results due to denominators $\frac{1}{t}$ or $\frac{1}{s+t}$ in $\left|\mathcal{M}_{i}\right|^{2}$. These divergences come from diagrams in which massless gluons are exchanged in the $t$ - or $u$-channel. The use of the exact integration limits eq. 2.17 does not help: it merely changes the integration limits by amounts of the order $m_{i}^{2}$, leading to logarithms of the order $\ln \frac{s}{m_{i}^{2}}$. The physical cut off however comes from temperature effects and not from the $T=0$ particle masses. Therefore the divergences must be regularized by introducing either a finite gluon mass $m_{\text {eff }}$ of the order $m_{\text {eff }} \sim g T$ or an angular cut around the forward and backward direction.

For the massive-gluon method, one has to recalculate the squared matrix elements using a modified denominator in the gluon propagator Feynman rule: $\frac{1}{K^{2}}$ becomes $\frac{1}{K^{2}-m_{\text {eff }}^{2}}(K$ is the gluon momentum). Provided that eq. 2.5 also holds for $m_{i}=m_{\text {eff }}$ (a somewhat questionable assumption), one is left to leading order with expressions that can be obtained from the old $\left|\mathcal{M}_{i}\right|^{2}$ by multiplying them with $\frac{t^{2}}{\left(t-m_{\text {eff }}^{2}\right)^{2}}$ in the case of processes $\mathrm{B}$, $\mathrm{G}$, and $\mathrm{H}$, and with $\frac{t^{2}}{\left(t-m_{\mathrm{eff}}^{2}\right)^{2}} \frac{(s+t)^{2}}{\left(s+t+m_{\text {eff }}^{2}\right)^{2}}$ for process $\mathrm{F}$. In the case of process B one obtains in addition a term proportional to $\frac{m_{\text {eff }}^{2}}{m_{\tilde{G}}^{2}}$ which is not shown in table 2.3 . With these new $\left|\mathcal{M}_{i}\right|^{2}$ the $t$-Integral is finite. As the leading term one obtains a logarithm $\ln \frac{s+m_{\text {eff }}^{2}}{m_{\text {eff }}^{2}}$. 


\begin{tabular}{c|c|l|l} 
& process $i$ & $\begin{array}{l}\sigma_{i} / F \text { with gluon-mass } \\
\text { regularization }\end{array}$ & $\begin{array}{l}\sigma_{i} / F \text { with angular-cut } \\
\text { regularization }\end{array}$ \\
\hline \hline $\mathrm{B}$ & $g^{a}+\tilde{g}^{b} \rightarrow g^{c}+\tilde{G}$ & $\left|f^{a b c}\right|^{2}\left(8 \ln \frac{s+m_{\text {eff }}^{2}}{m_{\text {eff }}^{2}}-14+\ldots\right)$ & $\left|f^{a b c}\right|^{2}\left(8 \ln \frac{2+\delta}{\delta}-6+\ldots\right)$ \\
\hline $\mathrm{F}$ & $\tilde{g}^{a}+\tilde{g}^{b} \rightarrow \tilde{g}^{c}+\tilde{G}$ & $\left|f^{a b c}\right|^{2}\left(16 \ln \frac{s+m_{\text {eff }}^{2}}{m_{\text {eff }}}-\frac{92}{3}+\ldots\right)$ & $\left|f^{a b c}\right|^{2}\left(16 \ln \frac{2+\delta}{\delta}-\frac{44}{3}+\ldots\right)$ \\
\hline $\mathrm{G}$ & $\tilde{g}^{a}+q_{i} \rightarrow q_{j}+\tilde{G}$ & $\left|T_{j i}^{a}\right|^{2}\left(4 \ln \frac{s+m_{\text {eff }}^{2}}{m_{\text {eff }}^{2}}-8+\ldots\right)$ & $\left|T_{j i}^{a}\right|^{2}\left(4 \ln \frac{2+\delta}{\delta}-4+\ldots\right)$ \\
\hline $\mathrm{H}$ & $\tilde{g}^{a}+\tilde{q}_{i} \rightarrow \tilde{q}_{j}+\tilde{G}$ & $\left|T_{j i}^{a}\right|^{2}\left(4 \ln \frac{s+m_{\text {eff }}^{2}}{m_{\text {eff }}^{2}}-7+\ldots\right)$ & $\left|T_{j i}^{a}\right|^{2}\left(4 \ln \frac{2+\delta}{\delta}-3+\ldots\right)$
\end{tabular}

Table 2.3: Total cross sections $\sigma_{i}$ up to a factor of $F=\frac{g^{2}}{16 \pi M^{2}}\left(1+\frac{m_{\tilde{g}}^{2}}{3 m_{\tilde{G}}^{2}}\right)$ for gravitino production. Logarithmic divergent processes; the cross sections are given for both gluonmass and angular-cut regularization, they are valid for the specified choice of colors and summed over spins in the initial and final state. As for chiral particles, only one chirality is included.

In the angular-cut method, one changes the integration variable from $t$ to $\cos \alpha$, where $\alpha$ is the scattering angle in the CMS system: $t=\frac{s}{2}(\cos \alpha-1), \frac{d \sigma}{d(\cos \alpha)}=\frac{s}{2} \frac{d \sigma}{d t}$. The integration is then not performed over the whole range $\cos \alpha=-1 \ldots 1$, but rather over a cut range $\cos \alpha=-1+\delta \ldots 1-\delta$ with a small parameter $\delta$. Again, one gets a logarithm $\ln \frac{2+\delta}{\delta}$ as the leading term.

Between the two cut-off parameters exists the relation

$$
\delta=2 \frac{m_{\mathrm{eff}}^{2}}{s}
$$

so the logarithms obtained from both cut-off methods are equivalent.

The total cross sections of the divergent processes B, F, G, and H are given in table 2.3 for both regularization methods. The logarithmically singular terms are universal whereas the finite parts depend on the cutoff procedure. The same result as with the angular-cutoff regularization is obtained from a naive gluon mass regularization, where one replaces $\frac{1}{t} \rightarrow \frac{1}{t-m_{\text {eff }}^{2}}$ and $\frac{1}{s+t} \rightarrow \frac{1}{s+t+m_{\text {eff }}^{2}}$ in $\left|\mathcal{M}_{i}\right|^{2}$ before doing the $t$-integral.

\subsection{The Boltzmann collision term}

We are interested in the cosmological production of gravitinos after an inflationary period which has practically erased any primordial gravitino abundance. As a thermal 
non-equilibrium process, gravitino production in the thermal bath is governed by the Boltzmann equation (cf. [25]):

$$
\frac{d n_{\tilde{G}}}{d t}+3 H n_{\tilde{G}}=\mathcal{C}
$$

Here, $n_{\tilde{G}}$ is the gravitino number density, $H$ is the Hubble constant, and $\mathcal{C}$ is the Boltzmann collision term. For the process $a+b \rightarrow c+\tilde{G}$ it reads

$$
\begin{aligned}
\mathcal{C}_{a+b \rightarrow c+\tilde{G}}= & \int d \Pi_{a} d \Pi_{b} d \Pi_{c} d \Pi_{\tilde{G}}(2 \pi)^{4} \delta\left(P_{a}+P_{b}-P_{c}-P_{\tilde{G}}\right) \times \\
& {\left[\left|\mathcal{M}_{\rightarrow}\right|^{2} f_{a} f_{b}\left(1 \pm f_{c}\right)\left(1-f_{\tilde{G}}\right)-\left|\mathcal{M}_{\leftarrow}\right|^{2} f_{c} f_{\tilde{G}}\left(1 \pm f_{a}\right)\left(1 \pm f_{b}\right)\right] }
\end{aligned}
$$

where

$$
d \Pi_{i}=\frac{d^{3} p_{i}}{(2 \pi)^{3} 2 E_{i}}
$$

(usually $d \Pi_{i}$ contains an additional factor $g_{i}$ that counts the spin degrees of freedom, but they are already summed over in my squared matrix elements), $\left|\mathcal{M}_{\rightarrow}\right|^{2}$ and $\left|\mathcal{M}_{\leftarrow}\right|^{2}$ are the squared matrix elements for the forward $(a+b \rightarrow c+\tilde{G})$ and backward $(c+\tilde{G} \rightarrow a+b)$ reaction, $f_{i}\left(E_{i}\right)$ are the phase space densities $\left(\int d \Pi_{i} g_{i} f_{i}\left(E_{i}\right)=n_{i}\right)$, and \pm applies for bosons/fermions.

Assuming CP invariance, one has $\left|\mathcal{M}_{\rightarrow}\right|^{2}=\left|\mathcal{M}_{\leftarrow}\right|^{2}=|\mathcal{M}|^{2}$. For the phase space densities $f_{a}, f_{b}$, and $f_{c}$, we take the equilibrium densities $f_{i}^{\mathrm{Eq}}$, i.e. a Bose or Fermi density $f_{B / F}=\left[\exp \left(E_{i} / T\right) \mp 1\right]^{-1}$, because in the temperature range of interest, all particles except gravitinos are in thermal equilibrium. In our case, the gravitino phase space density $f_{\tilde{G}}$ is so small that we can set $1-f_{\tilde{G}} \approx 1$ and neglect the backward reaction. So the form of the collision term we will work with is

$$
\mathcal{C}=\int d \Pi_{a} d \Pi_{b} d \Pi_{c} d \Pi_{\tilde{G}}(2 \pi)^{4} \delta\left(P_{a}+P_{b}-P_{c}-P_{\tilde{G}}\right)|\mathcal{M}|^{2} f_{a}^{\mathrm{Eq}} f_{b}^{\mathrm{Eq}}\left(1 \pm f_{c}^{\mathrm{Eq}}\right) .
$$

Now there are two possibilities to further proceed. In the usual treatment one also sets $1 \pm f_{c}^{\mathrm{Eq}} \approx 1$ before doing the integrations. I will review this method in the following. In appendix B.1 a formalism is presented in which this factor can be kept, because all phase space integrals are evaluated in the laboratory system. I will compare the results of both methods; the quantities resulting from the first method will be marked with a prime.

The factor $1 \pm f_{c}^{\mathrm{Eq}}$ depends explicitly on the energy $E_{c}$ of particle $c$ in the lab system (i.e. the rest system of the thermal bath), which is a non-covariant quantity. Without this factor the integration over the delta function can be done in the CMS system, leaving a differential cross section:

$$
\begin{aligned}
\mathcal{C}^{\prime} & =\int d \Pi_{a} d \Pi_{b} f_{a}^{\mathrm{Eq}} f_{b}^{\mathrm{Eq}} 4 I \int d \Pi_{c} d \Pi_{\tilde{G}}(2 \pi)^{4} \delta\left(P_{a}+P_{b}-P_{c}-P_{\tilde{G}}\right) \frac{|\mathcal{M}|^{2}}{4 I} \\
& =\int \frac{d^{3} p_{a}}{(2 \pi)^{3}} \frac{d^{3} p_{b}}{(2 \pi)^{3}} f_{a}^{\mathrm{Eq}} f_{b}^{\mathrm{Eq}} \frac{I}{E_{a} E_{b}} \int d t \frac{d \sigma_{i}}{d t}
\end{aligned}
$$


$\frac{I}{E_{a} E_{b}}$ is the relative velocity $v_{\text {rel }}$ of the incoming particles in the lab system. In the high energy limit,

$$
v_{\text {rel }}=1-\cos \theta
$$

where $\theta$ is the angle between $\mathbf{p}_{a}$ and $\mathbf{p}_{b}$. Note that the integrand of eq. 2.24 depends on four independent variables: $p_{a}, p_{b}, \cos \theta$ (these three fix $s$ in the lab system), and $t$.

If the total cross section $\sigma(s)$ is constant (i.e. independent of $s$ ), the angular integration gives just the usual factor of 2 , and the integration over the two three-momenta $p_{a}$ and $p_{b}$ of particles $a$ and $b$ gives together with this factor of 2 their equilibrium number densities $n_{a}^{\mathrm{Eq}} / g_{a}$ and $n_{b}^{\mathrm{Eq}} / g_{b}$ :

$$
\mathcal{C}^{\prime}=\frac{n_{a}^{\mathrm{Eq}}}{g_{a}} \frac{n_{b}^{\mathrm{Eq}}}{g_{b}} \sigma=\eta_{a} \eta_{b}\left(\frac{\zeta(3)}{\pi^{2}} T^{3}\right)^{2} \sigma,
$$

where $\eta_{i}=1$ for a boson and $\eta_{i}=\frac{3}{4}$ for a fermion.

If the total cross section $\sigma(s)$ depends on $s$, the integrations over the phase space factors are mixed with the one over $\sigma(s)$, and the equilibrium number densities do not arise naturally as factors in the result. However, one can still divide $\mathcal{C}^{\prime}$ by the factors $n_{a}^{\mathrm{Eq}} / g_{a}$ and $n_{b}^{\mathrm{Eq}} / g_{b}$. The remaining quantity is usually called the thermally averaged cross section $\Sigma^{\prime}(T)$ (it is often denoted $\Sigma^{\prime}(T)=\left\langle v_{\text {rel }} \sigma\right\rangle$ ), and one can write

$$
\mathcal{C}^{\prime}=\eta_{a} \eta_{b} n_{\text {rad }}^{2} \Sigma^{\prime}(T)
$$

with $n_{\text {rad }}=\frac{\zeta(3)}{\pi^{2}} T^{3}$ the number density of one bosonic relativistic degree of freedom.

Now let us calculate the Boltzmann collision term $\mathcal{C}_{2 \rightarrow 2}$ for gravitino production via inelastic $2 \rightarrow 2$ processes. The total cross sections for the ten processes A to J (tables 2.2 and 2.3) are to be summed over the colour degrees of freedom $(i, j, a)$ or $(a, b, c)$ and then multiplied with the following multiplicity factors ( $n_{f}$ is the number of quark flavors):

- $\frac{1}{2}$ for processes $\mathrm{A}$ and $\mathrm{F}$ : there are identical particles in the initial state.

- $2 n_{f}$ for processes I and J: $q_{i}$ (or $\tilde{q}_{i}$ ) can have $n_{f}$ flavors and two chiralities.

- $4 n_{f}$ for processes C, D, E, G, and H: $q_{i}$ (or $\tilde{q}_{i}$ ) can have $n_{f}$ flavors and two chiralities and can be particle or antiparticle.

For the actual calculation, one formally works in the limit of small coupling, $g \rightarrow 0$, and only in the end result one extrapolates to the real value of $g$. The leading term of $\mathcal{C}_{2 \rightarrow 2}$ comes from the logarithms in the total cross sections of processes B, F, G, and H. In [14] we have calculated this leading term of $\mathcal{C}_{2 \rightarrow 2}^{\prime}$. With $m_{\mathrm{eff}}^{2} \sim g^{2} T^{2}$ one has

$$
\ln \frac{s+m_{\mathrm{eff}}^{2}}{m_{\mathrm{eff}}^{2}} \approx \ln \frac{s}{m_{\mathrm{eff}}^{2}}=\ln \frac{1}{g^{2}}+\ln (1-\cos \theta)+\ln \frac{p_{a}}{T}+\ln \frac{p_{b}}{T}+\mathrm{cst} .
$$


The first term is the leading term. It is a constant, so one can apply eq. 2.26. With

$$
\begin{aligned}
\sum_{a, b, c}\left|f^{a b c}\right|^{2} & =C_{A}\left(N^{2}-1\right), \\
\sum_{i, j, a}\left|T_{j i}^{a}\right|^{2} & =C_{F} N \\
C_{A} & =N \\
C_{F} & =\frac{N^{2}-1}{2 N}
\end{aligned}
$$

for the group $\mathrm{SU}(\mathrm{N})\left(C_{A}\right.$ and $C_{F}$ are the usual group factors) one arrives at

$$
\mathcal{C}_{2 \rightarrow 2}^{\prime}=n_{\operatorname{rad}}^{2} \frac{g^{2}}{2 \pi M^{2}}\left(1+\frac{m_{\tilde{g}}^{2}}{3 m_{\tilde{G}}^{2}}\right)\left(N^{2}-1\right)\left(N+n_{f}\right)\left(\frac{21}{16} \ln \frac{1}{g^{2}}+\ldots\right) .
$$

This corresponds to the leading logarithmic term of our result eq. (4) in [14]. There we have used Maxwell-Boltzmann distributions rather than Bose or Fermi distributions for $f_{a}^{\mathrm{Eq}}$ and $f_{b}^{\mathrm{Eq}}$, this accounts for the difference of a factor of $1 / \zeta(3)^{2}$. The constant term in [14] comes from integrating over the next-to-leading terms in eq. 2.28.

A more exact result can be obtained when doing all phase space integrations in the lab system (see app. B.1), because then the quantum statistical factor $\left(1 \pm f_{c}^{\text {Eq }}\right)$ in eq. 2.23 can be kept. Picking out one of the six non-divergent processes A, C, D E, I, or J, and calculating their contribution $\mathcal{C}_{i}$ by doing the phase space integrations eq. B. 8 numerically one can determine the deviations from the the corresponding $\mathcal{C}_{i}^{\prime}$ obtained without the quantum statistical factor. For gravitino plus fermion in the final state, I found a decrease of $\mathcal{C}_{i}$ compared to $\mathcal{C}_{i}^{\prime}$ between $9 \%$ and $14 \%$. For gravitino plus boson in the final state, the $\mathcal{C}_{i}$ are $19 \%$ to $27 \%$ bigger than the corresponding $\mathcal{C}_{i}^{\prime}$.

For the divergent parts of the differential cross sections $\propto \frac{1}{t}$ and $\propto \frac{1}{u}$ which are responsible for the leading logarithmic term the phase space integrations can be done analytically in the weak coupling limit. The technical details are given in appendix B.2.

The result for the collision term of inelastic $2 \rightarrow 2$ gravitino production, calculated with the quantum statistical factors $\left(1 \pm f_{c}^{\mathrm{Eq}}\right)$ kept, is

$$
\mathcal{C}_{2 \rightarrow 2}=\left(N^{2}-1\right)\left(N+n_{f}\right)\left(1+\frac{m_{\tilde{g}}^{2}}{3 m_{\tilde{G}}^{2}}\right) \frac{T^{6}}{M^{2}} g^{2}\left(\frac{3 \zeta(3)}{32 \pi^{3}} \ln \frac{1}{g^{2}}+\ldots\right) .
$$

Compared to $\mathcal{C}_{2 \rightarrow 2}^{\prime}$ (eq. 2.30), the ratio of bosonic and fermionic contributions has changed from $1: \frac{3}{4}$ to $2: 1$, and the coefficient of the leading logarithmic term in $\mathcal{C}_{2 \rightarrow 2}$ is bigger by a factor of $\frac{\pi^{2}}{7 \zeta(3)}=1.173^{1}$.

\footnotetext{
${ }^{1}$ As a numerical effect, this is not very big, and it is mainly interesting because it yields analytically
} 
the same coefficient for the logarithmic term in $\mathcal{C}$ as from the gravitino self energy. However, inclusion of the quantum statistical factor of a final particle can have much more dramatic effects: consider the case where a particle species $X$ is produced mainly from decays $a \rightarrow b+X$. Then, if $b$ is a massless bosonic species in thermal equilibrium at a high temperature $T$, the $X$ production rate is enhanced by a factor of the order of $\ln \frac{T}{m_{a}}$. 


\section{Chapter 3}

\section{The Gravitino Self Energy}

In this chapter, the Boltzmann collision term for gravitino production is calculated in a second approach different from the one presented in the previous chapter: with the thermal version of the optical theorem. First, the relation between the imaginary part of a particle's self energy and its regeneration rate will be illustrated. Then the leading logarithmic and constant term of the imaginary part of the gravitino self energy will be calculated in the effective theory for light gravitinos with a resummed thermal gluon propagator.

\subsection{The optical theorem for finite temperature}

The optical theorem for zero temperature, as is well known, says that in a given process the imaginary part of the forward scattering amplitude is proportional to the total cross section (see, for example, [26]). For an unstable particle, one can thus relate the imaginary part of the self energy with its decay width.

Looking at a self energy with temperature switched on, a propagating particle can have interactions with other particles from the thermal plasma. Thus even stable particles can disappear, and they can also be produced, both by inelastic scattering processes with particles from the thermal background. Therefore at finite temperature the imaginary part of the self energy of a particle is not proportional to its decay rate, but rather to the rate of approaching a thermal equilibrium distribution, the so-called regeneration rate. This is explained in [27], and I will review some of this work now, especially the relation to the Boltzmann collision term. What follows will be a heuristic illustration of the general case, not a strict proof. The calculation in the specific case of the gravitino self energy in the effective theory for light gravitinos will be given with all technical details in section 3.2. 


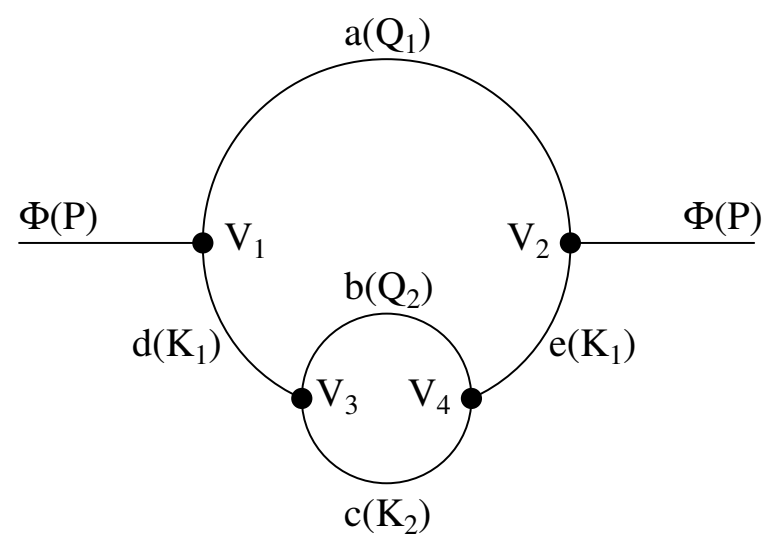

Figure 3.1: Two-loop self energy diagram of a boson $\Phi$

Consider the two-loop self energy diagram of a boson $\Phi$ fig. 3.1. Let us call it diagram (1) out of a complete set of $N$ two-loop self energy diagrams of the boson $\Phi$.

$\Phi$ has a four momentum $P=(E, \mathbf{p})$. The diagram has four vertices $V_{1}$ to $V_{4}$ which can depend on the momenta of all particles connected to the vertex: $V_{i}=V_{i}\left(P_{j}\right)$, where $P_{j}$ stands for three four momenta. The loop momenta to be integrated over are $K_{1}=\left(k_{10}, \mathrm{k}_{1}\right)$ for the particles $d$ and $e$, and $K_{2}=\left(k_{20}, \mathrm{k}_{2}\right)$ for particle $c$. The other particles in the loops carry momenta $Q_{1}=P-K_{1}$ (particle $a$ ) and $Q_{2}=K_{1}-K_{2}$ (particle $b$ ). For simplicity, all particles in the loops are assumed to be bosons, too. We are interested in the imaginary part of the diagram associated with the three particle state consisting of $a, b$, and $c$.

The diagram represents the self energy contribution

$$
\Sigma_{\Phi}^{(1)}(P)=T^{2} \sum_{k_{10}, k_{20}} \int \frac{d^{3} k_{1}}{(2 \pi)^{3}} \frac{d^{3} k_{2}}{(2 \pi)^{3}} V_{1} \Delta_{d}\left(K_{1}\right) V_{3} \Delta_{a}\left(Q_{1}\right) \Delta_{b}\left(Q_{2}\right) \Delta_{c}\left(K_{2}\right) V_{4} \Delta_{e}\left(K_{1}\right) V_{2}
$$

the $\Delta_{i}$ 's denote propagators.

Now it is argued in [27] that the imaginary part associated with the three particles $a, b$, and $c$ comes from the three propagators $\Delta_{a}\left(Q_{1}\right), \Delta_{b}\left(Q_{2}\right)$, and $\Delta_{c}\left(K_{2}\right)$, and not from the vertices or from other propagators. Therefore one may replace in the vertices $V_{i}\left(P_{j}\right)$ the zero components $p_{j 0}$ of the momenta (which initially are summed over discrete imaginary values) by the energies $E_{j}=\sqrt{p_{j}^{2}+m_{j}^{2}}$. The same holds for $k_{10}$ in the two propagators $\Delta_{d}\left(K_{1}\right)$ and $\Delta_{e}\left(K_{1}\right)$, and one is left with the product of two ordinary zero-temperature amplitudes $A^{(1)}\left(p, k_{1}, k_{2}\right) B^{(1)}\left(p, k_{1}, k_{2}\right)$ which is a part of the the tree level order of the 
squared matrix element $\left|\mathcal{M}_{\Phi a b c}^{\text {tree }}\right|^{2}$ for reactions between the particles $\Phi, a, b$, and $c$ :

$$
\Sigma_{\Phi}^{(1)}(P)=T^{2} \sum_{k_{10}, k_{20}} \int \frac{d^{3} k_{1}}{(2 \pi)^{3}} \frac{d^{3} k_{2}}{(2 \pi)^{3}} A^{(1)} B^{(1)} \Delta_{a}\left(Q_{1}\right) \Delta_{b}\left(Q_{2}\right) \Delta_{c}\left(K_{2}\right) .
$$

Using the Saclay representation of the propagators and doing the sums over Matsubara frequencies (see section 3.2) gives

$$
\Sigma_{\Phi}^{(1)}(P)=-\int \frac{d^{3} k_{1}}{(2 \pi)^{3}} \frac{d^{3} k_{2}}{(2 \pi)^{3}} A^{(1)} B^{(1)} \int_{0}^{\beta} d \tau e^{E \tau} \prod_{p_{i}=q_{1}, q_{2}, k_{2}} \frac{1}{2 E_{i}} \sum_{\sigma_{i}= \pm 1} e^{\sigma_{i} E_{i} \tau} g_{i}^{\sigma_{i}}\left(E_{i}\right)
$$

with the statistical factors $g^{ \pm}$for bosons

$$
g^{+}(E)=f_{B}(E), \quad g^{-}(E)=1+f_{B}(E) .
$$

After $\tau$ integration and replacing $e^{E \beta}=1$ one has

$$
\Sigma_{\Phi}^{(1)}(P)=-\int \frac{d^{3} k_{1}}{(2 \pi)^{3}} \frac{d^{3} k_{2}}{(2 \pi)^{3}} \frac{A^{(1)} B^{(1)}}{8 E_{q_{1}} E_{q_{2}} E_{k_{2}}} \sum_{j=1}^{8} \frac{e^{S_{j} \beta}-1}{E+S_{j}} g_{1}^{\sigma_{1}}\left(E_{q_{1}}\right) g_{2}^{\sigma_{2}}\left(E_{q_{2}}\right) g_{3}^{\sigma_{3}}\left(E_{k_{2}}\right),
$$

where $j$ counts the eight possible sign combinations $\sigma_{1} \sigma_{2} \sigma_{3}$, and $S_{j}$ are the corresponding energy sums $S_{j}=\sigma_{1} E_{q_{1}}+\sigma_{2} E_{q_{2}}+\sigma_{3} E_{k_{2}}$. The statistical factors can be combined with the exponentials using the relation $e^{\sigma_{i} E_{i} \beta} g_{i}^{\sigma_{i}}\left(E_{i}\right)=g_{i}^{-\sigma_{i}}\left(E_{i}\right)$. With the identity

$$
\lim _{\varepsilon \rightarrow 0} \Im\left(\frac{1}{E+i \varepsilon+X}\right)=-\pi \delta(E+X)
$$

one gets the imaginary part

$$
\Im\left[\Sigma_{\Phi}^{(1)}(E+i \varepsilon, p)\right]=\pi \int \frac{d^{3} k_{1}}{(2 \pi)^{3}} \frac{d^{3} k_{2}}{(2 \pi)^{3}} \frac{A^{(1)} B^{(1)}}{8 E_{q_{1}} E_{q_{2}} E_{k_{2}}} \sum_{j=1}^{8}\left(G_{P j}-G_{D j}\right) \delta\left(E+S_{j}\right) .
$$

Here the $G_{P j}$ and $G_{D j}$ are products of statistical factors that will turn out to belong to $\Phi$ production and $\Phi$ disappearance processes:

$$
\begin{aligned}
& G_{P j}=g_{1}^{-\sigma_{1}}\left(E_{q_{1}}\right) g_{2}^{-\sigma_{2}}\left(E_{q_{2}}\right) g_{3}^{-\sigma_{3}}\left(E_{k_{2}}\right), \\
& G_{D j}=g_{1}^{\sigma_{1}}\left(E_{q_{1}}\right) g_{2}^{\sigma_{2}}\left(E_{q_{2}}\right) g_{3}^{\sigma_{3}}\left(E_{k_{2}}\right) .
\end{aligned}
$$

Other two-loop diagrams than the one shown in figure 3.1 contribute other products of amplitudes $A^{(n)} B^{(n)}$. By summing up a certain subset (let's call it the $a b c$ subset) of all $N$ two-loop self energy diagrams of $\Phi$ one can collect all terms $A^{(n)} B^{(n)}$ which give together the squared matrix element $\left|\mathcal{M}_{\Phi a b c}^{\text {tree }}\right|^{2}$. Calling the self energy resulting from this subset $\Sigma_{\Phi}^{a b c}$, let us look at a particular $S_{j}$, say $S_{4}=E_{q_{1}}-E_{q_{2}}-E_{k_{2}}$. Writing

$$
\int \frac{d^{3} k_{1}}{(2 \pi)^{3}}=\int \frac{d^{3} q_{1}}{(2 \pi)^{3}} \frac{d^{3} q_{2}}{(2 \pi)^{3}}(2 \pi)^{3} \delta\left(\mathbf{p}+\mathbf{q}_{1}-\mathbf{q}_{2}-\mathbf{k}_{2}\right)
$$


for $j=4$ one gets the contribution (possibly after a redefinition of the three momentum flow)

$$
\begin{aligned}
& \Im\left[\Sigma_{\Phi}^{a b c}(E+i \varepsilon, p)\right]_{j=4}= \\
& \quad=\frac{1}{2} \int d \Pi_{q_{1}} d \Pi_{q_{2}} d \Pi_{k_{2}}\left|\mathcal{M}_{\Phi a b c}^{\mathrm{tree}}\right|^{2}(2 \pi)^{4} \delta\left(P+Q_{1}-Q_{2}-K_{2}\right)\left(G_{P 4}-G_{D 4}\right)
\end{aligned}
$$

with

$$
\begin{aligned}
G_{P 4} & =\left[1+f_{B}\left(E_{q_{1}}\right)\right] f_{B}\left(E_{q_{2}}\right) f_{B}\left(E_{k_{2}}\right), \\
G_{D 4} & =f_{B}\left(E_{q_{1}}\right)\left[1+f_{B}\left(E_{q_{2}}\right)\right]\left[1+f_{B}\left(E_{k_{2}}\right)\right] .
\end{aligned}
$$

Written in this form it is clearly apparent that the term proportional to $G_{P 4}$ in eq. 3.10 is $E$ times the $\Phi$ production rate $\Gamma_{P 4}^{a b c}$ in a thermal plasma through the process $b+c \rightarrow a+\Phi$; the term proportional to $G_{D 4}$ is $E$ times the $\Phi$ disappearance rate $\Gamma_{D 4}^{a b c}$ trough the inverse process:

$$
\Im\left[\Sigma_{\Phi}(E+i \varepsilon, p)\right]_{j=4}=E\left(\Gamma_{P 4}^{a b c}-\Gamma_{D 4}^{a b c}\right)=-E \Gamma_{\Phi 4}^{a b c} .
$$

Here the $\Phi$ regeneration rate $\Gamma_{\Phi 4}^{a b c}$ was introduced, the meaning of which will become clear soon. Note that

$$
\frac{\Gamma_{D j}^{a b c}}{\Gamma_{P j}^{a b c}}=\frac{G_{D j}}{G_{P j}}=e^{-S_{j} \beta}=e^{E \beta} ;
$$

this ratio is a universal function of $E$, independent of the other energies.

The eight possible sign combinations $\sigma_{1} \sigma_{2} \sigma_{3}, j=1 \ldots 8$, represent $\Phi$ production and $\Phi$ disappearance through the following eight processes and their inverse processes:

$$
\begin{array}{lrl}
j=1(+++): & \text { nothing } & \rightarrow \Phi+a+b+c, \\
j=2(++-): & c & \rightarrow \Phi+a+b, \\
j=3(+-+): & b & \rightarrow \Phi+a+c, \\
j=4(+--): & a+c \rightarrow \Phi+a, \\
j=5(-++): & a+b \rightarrow \Phi+c \rightarrow c, \\
j=6(-+-): & a+b+c \rightarrow \Phi .
\end{array}
$$

Process $j=1$ is never possible; the corresponding delta function in eq. 3.7 will always be zero. Of the four $1 \rightarrow 3$ decay processes $j=2,3,5,8$ and their inverse counterparts 
one is possible if the mass of one of the four particles $\Phi, a, b$, and $c$ is bigger than the sum of the masses of the three remaining particles, otherwise none of these processes is possible. The three $2 \rightarrow 2$ processes $j=4,6,7$ are possible for arbitrary particle masses. The individual $\Gamma_{D j}^{a b c}, \Gamma_{P j}^{a b c}$ sum up to $\Gamma_{D}^{a b c}, \Gamma_{P}^{a b c}$. Here one sees that in the imaginary part of the self energy, all three or four reaction channels of $\left|\mathcal{M}_{\Phi a b c}^{\text {tree }}\right|^{2}$ are taken into account according to their range of definition in terms of the particle momenta.

By integrating the rates $\Gamma_{D}^{a b c}$ and $\Gamma_{P}^{a b c}$ over the $\Phi$ three momentum one can build a Boltzmann collision term for the time development of the $\Phi$ number density $n_{\Phi}$ (cf. eq. 2.21) resulting from reactions with the particles $a, b$, and $c\left(f_{\Phi}(E)\right.$ is the distribution function of the $\Phi$ particles):

$$
\mathcal{C}_{a b c}^{\text {tree }}=\int \frac{d^{3} p}{(2 \pi)^{3}}\left[\left(1+f_{\Phi}\right) \Gamma_{P}^{a b c}-f_{\Phi} \Gamma_{D}^{a b c}\right] .
$$

Eq. 3.12 and eq. 3.13 yield

$$
\Gamma_{D}=\left(1+f_{B}\right) \Gamma_{\Phi}, \quad \Gamma_{P}=f_{B} \Gamma_{\Phi}
$$

With this, eq. 3.15 simplifies to

$$
\mathcal{C}_{a b c}^{\text {tree }}=\int \frac{d^{3} p}{(2 \pi)^{3}}\left(f_{B}-f_{\Phi}\right) \Gamma_{\Phi}^{a b c} .
$$

Other subsets of two-loop self energy diagrams than the $(a b c)$ subset will yield tree level squared matrix elements $\left|\mathcal{M}_{i}\right|^{2}$ of other $\Phi$ production or $\Phi$ disappearance processes involving four particles, or one-loop corrections to $1 \rightarrow 2$ or $2 \rightarrow 1$ processes. The imaginary part of the one-loop self energy gives a Boltzmann collision term associated with tree-level $1 \rightarrow 2$ or $2 \rightarrow 1$ processes. In general, $L$-loop self energy diagrams give Boltzmann collision terms for $\Phi$ production or $\Phi$ disappearance processes involving $(L+2)$ particles on tree level, or $l$-loop corrections to processes involving $(L+2-l)$ particles.

Eq. 3.17 is the desired relation between the imaginary part of the self energy of a boson and the Boltzmann collision term for the time evolution of its number density. However, it seems difficult to establish in an actual calculation a one to one relation between the Boltzmann collision term resulting from one particular $2 \rightarrow 2$ process and one particular two-loop self energy diagram, because the reaction channels of the matrix element summed over in the self energy correspond to $2 \rightarrow 2$ Feynman diagrams of different processes. A simple relation exists only between the Boltzmann collision term resulting from all relevant production and disappearance processes, and the imaginary part of the complete self energy to a given order.

In order to include the case of a fermionic $\Phi$, one has to generalize the definition of the regeneration rate $\Gamma_{\Phi}$ to

$$
\Gamma_{\Phi}=\Gamma_{D}-s_{\Phi} \Gamma_{P}
$$


(where $s_{\Phi}= \pm 1$ for a boson/fermion), because for fermionic $\Phi$ one finds

$$
-\frac{\Im\left(\bar{\Psi}_{\Phi} \Sigma_{\Phi} \Psi_{\Phi}\right)}{E}=\Gamma_{D}+\Gamma_{P},
$$

( $\Psi_{\Phi}$ is the $\Phi$ wave function), and the Boltzmann collision term is given by

$$
\mathcal{C}_{\Phi}=\int \frac{d^{3} p}{(2 \pi)^{3}}\left(f_{F}-f_{\Phi}\right) \Gamma_{\Phi}
$$

The reason for the two different forms of the regeneration rate $\Gamma_{\Phi}$ for a bosonic and for a fermionic $\Phi$ will become clear if we take a look at its meaning. Consider an arbitrary energy distribution of $\Phi$ particles $f_{\Phi}(E)$. It follows a time evolution (neglecting the expansion of the universe)

$$
\frac{\partial f_{\Phi}(E, t)}{\partial t}=-f_{\Phi}(E, t) \Gamma_{D}+\left[1+s_{\Phi} f_{\Phi}(E, t)\right] \Gamma_{P}
$$

For small deviations from equilibrium, eq. 3.21 has the solution

$$
f_{\Phi}(E, t)=\frac{\Gamma_{P}}{\Gamma_{D}-s_{\Phi} \Gamma_{P}}+c(E) e^{-\left(\Gamma_{D}-s_{\Phi} \Gamma_{P}\right) t}=\frac{1}{e^{e \beta}-s_{\Phi}}+c(E) e^{-\Gamma_{\Phi} t},
$$

where $c(E)$ is an arbitrary function. For the last identity, eq. 3.13 was used. Eq. 3.22 means that the final form of $f_{\Phi}(E, t)$ is a Bose or Fermi distribution, and that a departure from this equilibrium distribution is washed out with the regeneration rate $\Gamma_{\Phi}$. For bosons, the regeneration rate is the difference between decay rate and production rate, whereas for fermions it is their sum.

\subsection{The gravitino regeneration rate}

Having established the relation between the imaginary part of a particle's self energy and the Boltzmann term of its production, let us use this formalism to calculate the Boltzmann collision term for gravitino production in a second way, using the effective theory for light gravitinos.

Compared to calculating the Boltzmann collision term $\mathcal{C}_{2 \rightarrow 2}$ arising from individual $2 \rightarrow 2$ production processes, as it was done in chapter 2 , the self energy formalism has three advantages: one can clarify the nature of the divergence in the total cross sections, one can obtain the constant term, and one can be sure that all relevant processes are automatically taken into account.

First, the self energy is a quantity which adapts itself to a calculation in the framework of thermal field theory. Here a resummed gluon propagator which contains contributions 
from an arbitrary number of hard thermal loops is used instead of the ordinary $T=0$ gluon propagator. The calculation in this framework will turn out to give a finite result; the divergence from exchanging soft gluons in the $t$ or $u$ channel is regularized by the electric Debye mass of the gluon. If the result were divergent, this would mean that the cut-off comes from the magnetic Debye mass of the gluon.

Second, since in the self energy formalism one has a consistent regularization procedure rather than a cut-off by an ad hoc introduced effective gluon mass of which only the order of magnitude is known, one cannot only calculate the leading logarithmic term but also the corresponding constant term of the Boltzmann collision term.

Third, the imaginary part of the self energy takes into account all relevant production processes, no matter how many particles are involved. So if one has the self energy to a given order in the coupling constant, one can be sure that no production or disappearance process contributing to this order has been overlooked. In turn, the fact that with the self energy method we will find a Boltzmann collision term $\mathcal{C}_{\Sigma}$ equal to $\mathcal{C}_{2 \rightarrow 2}$ eq. 2.31, is a proof that the ten $2 \rightarrow 2$ processes from section 2.2 are really the dominant gravitino production processes, and that $1 \rightarrow 2$ decays or processes with more than four particles involved can be neglected.

On the one-loop level, there are two diagrams which contribute to the gravitino self energy (see fig. 3.2): one with a gluon-gluino loop (a) and one with a quark-squark loop (b). As

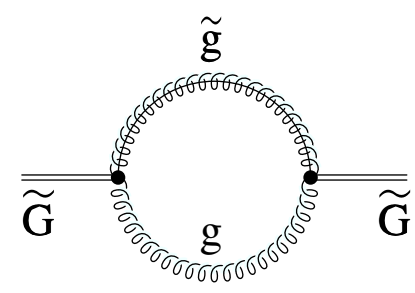

(a)

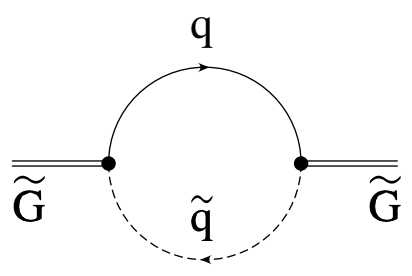

(b)

Figure 3.2: Feynman diagrams contributing to the gravitino self energy on the one-loop level

we have seen, the leading logarithmic terms in the Boltzmann collision term $\mathcal{C}_{2 \rightarrow 2}$ from $2 \rightarrow 2$ processes all come from $t$ - or $u$-channel gluon exchange diagrams. These diagrams correspond to the gravitino self energy diagram (a). Furthermore, diagram (b) cannot contribute to the leading logarithmic or to the corresponding constant term, because it contains two gravitino-quark-squark vertices which each contribute a factor of $\frac{m_{q}^{2}-m_{\tilde{q}}^{2}}{M_{\tilde{G}}}$, whereas the gravitino-gluon-gluino vertices of diagram (a) are $\propto \frac{m_{\tilde{g}}}{M m_{\tilde{G}}}$ which is one mass 
factor less. Since no massless particles are exchanged, we do not expect any logarithmic terms from diagram (b). Thus the gravitino regeneration rate resulting from diagram (b) will be down by a factor of $\frac{m_{\tilde{\tilde{q}}}^{2}}{T^{2}}$ (assuming $m_{\tilde{q}} \sim m_{\tilde{g}}$ ) compared to the constant term resulting from diagram (a). Therefore we can restrict ourselves to calculating only diagram (a).

At $T=0$, diagram (a) represents the expression

$$
S(P):=\operatorname{tr}\left[\psi(P) \Sigma_{\tilde{G}} \bar{\psi}(P)\right]=i \int \frac{d^{4} K}{(2 \pi)^{4}} \operatorname{tr}\left[\left(\not P+m_{\tilde{G}}\right) V_{2}^{\nu} i \tilde{S}(Q) i S_{\mu \nu}(K) V_{1}^{\mu}\right] .
$$

Here, $V_{1}^{\mu}$ and $V_{2}^{\nu}$ are the vertices, $i \tilde{S}(Q)$ is the gluino propagator, and $i S_{\mu \nu}(K)$ is the gluon propagator (cf. fig. 3.3). $P=(E, \mathbf{p}), K=\left(k_{0}, \mathbf{k}\right)$, and $Q=P-K=\left(q_{0}, \mathbf{q}\right)$ are the gravitino, gluon, and gluino four momenta.

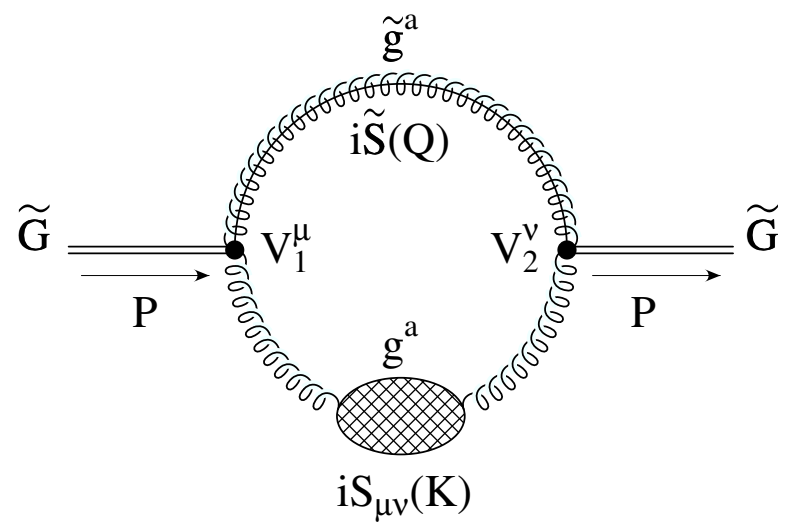

Figure 3.3: gluon-gluino loop diagram, the leading contribution to the imaginary part of the gravitino self energy. The blob denotes a resummed gluon propagator.

We will carry out the calculation in the imaginary-time formalism of thermal field theory. As is well-known, for gluon momenta of the order $g T$ ("soft" momenta), loop corrections to the gluon propagator become as large as the bare propagator itself, and the same can happen with vertex corrections that would be of higher order at $T=0$. Therefore one has to reorganize the perturbation series. A consistent resummation is done in the hard thermal loop (HTL) approach [28], where one uses a resummed propagator whenever the particle carries soft momentum, and a resummed vertex whenever all momenta going into the vertex are soft. In this context, a momentum $P=(E, \mathbf{p})$ is termed soft if both $E$ and $p$ are at most of order $g T$. Otherwise, $P$ is hard.

Since the gravitino momentum $P$ is of order $T$, we do not need any resummed vertices. The zero component of the fermion four momentum in the loop is summed over odd 
Matsubara frequencies of which the smallest is $i \pi T$, so these momenta are always hard and we don't need resummed fermion propagators either. The gluon momentum however can become soft, therefore we need a resummed gluon propagator for soft gluon three momenta $k$. In [29] a similar calculation, the production of axions in a hot plasma, is divided into two parts: contributions from soft gluons are obtained from the imaginary part of the axion self energy with a resummed gluon propagator, whereas contributions from hard gluons are calculated from the matrix element for the $2 \rightarrow 2$ axion production process with a bare gluon propagator. The division line is a gluon three momentum $\bar{k}$ of the order of $\sqrt{g} T$. Both contributions match in a way that $\bar{k}$ cancels out in the end result. Here, I will use a resummed gluon propagator for both soft and hard gluons, but for technical reasons I will keep the division into the two regimes.

The resummed gluon propagator reads [30, 31]

$$
i S_{\mu \nu}(K)=i\left(A_{\mu \nu} \Delta_{T}+B_{\mu \nu} \Delta_{L}+C_{\mu \nu} \xi\right)
$$

with the tensors ( $v$ is the velocity of the thermal bath)

$$
\begin{aligned}
A_{\mu \nu} & =-g_{\mu \nu}-\frac{1}{k^{2}}\left[K^{2} v_{\mu} v_{\nu}-K \cdot v\left(K_{\mu} v_{\nu}+K_{\nu} v_{\mu}\right)+K_{\mu} K_{\nu}\right], \\
B_{\mu \nu} & =v_{\mu} v_{\nu}-\frac{K \cdot v}{K^{2}}\left(K_{\mu} v_{\nu}+K_{\nu} v_{\mu}\right)+\left(\frac{K \cdot v}{K^{2}}\right)^{2} K_{\mu} K_{\nu}, \\
C_{\mu \nu} & =\frac{K_{\mu} K_{\nu}}{\left(K^{2}\right)^{2}}
\end{aligned}
$$

and the transverse and longitudinal gluon propagators

$$
\begin{aligned}
\Delta_{T}\left(k_{0}, k\right) & =\frac{1}{k_{0}^{2}-k^{2}-\Pi_{T}\left(k_{0}, k\right)}, \\
\Delta_{L}\left(k_{0}, k\right) & =\frac{1}{k^{2}-\Pi_{L}\left(k_{0}, k\right)} .
\end{aligned}
$$

$\Pi_{T}$ and $\Pi_{L}$ are the transverse and longitudinal gluon self energies:

$$
\begin{aligned}
& \Pi_{T}\left(k_{0}, k\right)=\frac{3}{2} m_{g}^{2}\left[\frac{k_{0}^{2}}{k^{2}}-\frac{k_{0}}{2 k}\left(\frac{k_{0}^{2}}{k^{2}}-1\right) \ln \frac{k_{0}+k}{k_{0}-k}\right], \\
& \Pi_{L}\left(k_{0}, k\right)=\frac{3}{2} m_{g}^{2}\left[\frac{k_{0}}{k} \ln \frac{k_{0}+k}{k_{0}-k}-2\right] .
\end{aligned}
$$

Unlike in [30], here we have to use the supersymmetric effective gluon mass

$$
m_{g}^{2}=\left(m_{g}^{\mathrm{MSSM}}\right)^{2}=\frac{g^{2} T^{2}}{6}\left(N+n_{f}\right)
$$


which is derived in appendix $\mathrm{C}$.

The other ingredients of expression $S$ (eq. 3.23) are given in appendix A, and we get

$$
S=-i C \int \frac{d^{4} K}{(2 \pi)^{4}}\left(D_{L} \Delta_{L}+D_{T} \Delta_{T}\right) \frac{1}{Q^{2}-m_{\tilde{g}}^{2}}
$$

with the constant factor

$$
C=\frac{4}{3} \frac{m_{\tilde{g}}^{2}}{M^{2} m_{\tilde{G}}^{2}}\left(N^{2}-1\right)
$$

(the factor $N^{2}-1$ comes from summing over the gluonic colour degrees of freedom) and the Dirac traces

$$
\begin{aligned}
& D_{T}\left(k_{0}, k, E, p, \text { pk }\right)=\frac{1}{32} \operatorname{tr}\left\{\left(\not P+m_{\tilde{G}}\right)\left[K K, \gamma^{\nu}\right]\left(\not Q+m_{\tilde{g}}\right)\left[\not K, \gamma^{\mu}\right] A_{\mu \nu}\right\}, \\
& D_{L}\left(k_{0}, k, E, p, \text { pk }\right)=\frac{1}{32} \operatorname{tr}\left\{\left(\not P+m_{\tilde{G}}\right)\left[K K, \gamma^{\nu}\right]\left(\not+m_{\tilde{g}}\right)\left[\not K, \gamma^{\mu}\right] B_{\mu \nu}\right\} .
\end{aligned}
$$

These traces are taken in Minkowski space time. Note that the term proportional to the gauge parameter $\xi$ disappears, because each gluon momentum in $C_{\mu \nu}$ is contracted with a commutator containing another gluon momentum from the gravitino-gluon-gluino vertex, yielding zero: $S$ is a gauge-independent quantity.

Now we switch to Euklidean space time, giving the zero components of the four momenta discrete imaginary values. The integral $\int \frac{d^{4} K}{(2 \pi)^{4}}$ becomes a sum integral $i T \sum_{k_{0}} \int \frac{d^{3} k}{(2 \pi)^{3}}$, and the propagator denominators are replaced with their Saclay representations [32]:

$$
\begin{aligned}
\frac{1}{Q^{2}-m_{\tilde{g}}^{2}} & =-\int_{0}^{\beta} d \tau e^{q_{0} \tau} \tilde{\Delta}\left(E_{q}, \tau\right) \\
\tilde{\Delta}\left(E_{q}, \tau\right) & =\frac{1}{2 E_{q}}\left\{\left[1-f_{F}\left(E_{q}\right)\right] e^{-E_{q} \tau}-f_{F}\left(E_{q}\right) e^{E_{q} \tau}\right\}
\end{aligned}
$$

(with $E_{q}=\sqrt{q^{2}+m_{\tilde{g}}^{2}}$ ) and

$$
\begin{aligned}
& \Delta_{T / L}\left(k_{0}, k\right)=-\int_{0}^{\beta} d \tau^{\prime} e^{k_{0} \tau^{\prime}} \Delta_{T / L}\left(k, \tau^{\prime}\right) \\
& \Delta_{T / L}\left(k, \tau^{\prime}\right)=\int_{-\infty}^{\infty} d \omega\left[1+f_{B}(\omega)\right] e^{-\omega \tau^{\prime}} \rho_{L / T}(\omega, k)
\end{aligned}
$$

with the gluon spectral densities [31]

$$
\begin{aligned}
\rho_{T}(\omega, k) & =\frac{3}{4} m_{g}^{-2} \frac{x /\left(1-x^{2}\right)}{A_{T}^{2}+\left(z+B_{T}\right)^{2}} \theta(|x|-1)+\rho_{T}^{P}(\omega, k), \\
\rho_{L}(\omega, k) & =\frac{3}{4} m_{g}^{-2} \frac{2 x}{A_{L}^{2}+\left(z+B_{L}\right)^{2}} \theta(|x|-1)+\rho_{L}^{P}(\omega, k) .
\end{aligned}
$$


Here, $x=\omega / k, z=k^{2} / m_{g}^{2}$,

$$
\begin{aligned}
& A_{T}(x)=\frac{3}{4} \pi x, \quad B_{T}(x)=\frac{3}{4}\left(2 \frac{x^{2}}{1-x^{2}}+x \ln \frac{1+x}{1-x}\right), \\
& A_{L}(x)=\frac{3}{2} \pi x, \quad B_{L}(x)=\frac{3}{2}\left(2-x \ln \frac{1+x}{1-x}\right),
\end{aligned}
$$

and the delta function contributions

$$
\rho_{T / L}^{P}(\omega, k)=\operatorname{Res}_{T / L}\left(\omega_{T / L}, k\right)\left[\delta\left(\omega-\omega_{T / L}\right)-\delta\left(\omega+\omega_{T / L}\right)\right]
$$

from the poles $\omega_{T / L}(k)$ of the propagators $\Delta_{T / L}\left(\operatorname{Res}_{T / L}\right.$ are the residues of the poles). These delta function parts of the gluon spectral densities, however, will not contribute to the leading order terms of $S$ (see appendix D.1).

With these replacements, our expression reads

$$
S=C T \sum_{k_{0}} \int \frac{d^{3} k}{(2 \pi)^{3}} \int_{0}^{\beta} d \tau d \tau^{\prime} \int_{-\infty}^{\infty} d \omega e^{q_{0} \tau+k_{0} \tau^{\prime}} e^{-\omega \tau^{\prime}} \tilde{\Delta}\left(E_{q}, \tau\right)\left[1+f_{B}(\omega)\right]\left(D_{T} \rho_{T}+D_{L} \rho_{L}\right) .
$$

Next we eliminate factors $k_{0}^{n}$ in the Dirac traces $D_{T / L}$ by writing $k_{0} e^{k_{0} \tau^{\prime}}=\frac{\partial}{\partial \tau^{\prime}} e^{k_{0} \tau^{\prime}}$ and integrating by parts: the border term gives zero, and the remaining integral is just the initial expression with $k_{0}$ replaced by $\omega$. This can be done for arbitrary powers $k_{0}^{n}$, so we get

$$
D_{T / L}\left(k_{0}, k, E, p, \mathbf{p k}\right) \rightarrow D_{T / L}(\omega, k, E, p, \mathbf{p k}) .
$$

Now the summation over $k_{0}$ can be performed,

$$
T \sum_{k_{0}} e^{k_{0}\left(\tau-\tau^{\prime}\right)}=\delta\left(\tau-\tau^{\prime}\right)
$$

The integration over $\tau^{\prime}$ is trivial, and after $\tau$ integration we are left with

$$
\begin{aligned}
S(E, p)= & C \int \frac{d^{3} k}{(2 \pi)^{3}} \int_{-\infty}^{\infty} d \omega\left(D_{T} \rho_{T}+D_{L} \rho_{L}\right) \frac{1+f_{B}(\omega)}{2 E_{q}} \times \\
& \times\left\{\frac{e^{\left(E-\omega-E_{q}\right) \beta}-1}{E-\omega-E_{q}}\left[1-f_{F}\left(E_{q}\right)\right]-\frac{e^{\left(E-\omega+E_{q}\right) \beta}-1}{E-\omega+E_{q}} f_{F}\left(E_{q}\right)\right\} .
\end{aligned}
$$

Here, the gravitino energy $E$ takes values $E=(2 n+1) i \pi T$, therefore $e^{E \beta}=-1$. After this replacement, we extend $S(E, p)$ to the whole complex plain. In order to get the imaginary part $\Im[S(E+i \varepsilon, p)]$ we use the identity eq. 3.6.

After simplifying the products of the statistical factors $f_{B / F}$ and the exponentials one has

$$
\begin{aligned}
\Im(S)= & \frac{C}{8 \pi} \int d k d \eta d \omega \frac{k^{2}}{E_{q}}\left(D_{T} \rho_{T}+D_{L} \rho_{L}\right) \times \\
& \times\left[\delta\left(E-\omega-E_{q}\right)\left(1+f_{\omega}-f_{q}\right)-\delta\left(E-\omega+E_{q}\right)\left(f_{\omega}+f_{q}\right)\right],
\end{aligned}
$$


where $f_{\omega}$ and $f_{q}$ stand for $f_{B}(\omega)$ and $f_{F}\left(E_{q}\right)$, and $\eta$ is the cosine of the angle between $\mathbf{p}$ and $\mathrm{k}$.

The angular integration produces a factor of $\frac{E_{q}}{k p}$ and determines the scalar product pk: for both delta functions, one has

$$
\mathrm{pk}=E \omega+\frac{1}{2}\left(k^{2}-\omega^{2}+m_{\tilde{g}}^{2}-m_{\tilde{G}}^{2}\right) .
$$

Furthermore, the $\omega$ integration region is now restricted: only for the two ranges

$$
\begin{aligned}
& \omega=E-\sqrt{(p+k)^{2}+m_{\tilde{g}}^{2}} \ldots E-\sqrt{(p-k)^{2}+m_{\tilde{g}}^{2}}, \\
& \omega=E+\sqrt{(p-k)^{2}+m_{\tilde{g}}^{2}} \ldots E+\sqrt{(p+k)^{2}+m_{\tilde{g}}^{2}}
\end{aligned}
$$

the delta functions can be satisfied for some value of $\eta$ between -1 and 1 , and from the requirement that $E_{q}$ be positive a theta function is left over from each delta function:

$$
\begin{aligned}
\Im(S)= & \frac{C}{8 \pi p} \int d k d \omega k\left(D_{T} \rho_{T}+D_{L} \rho_{L}\right) \times \\
& \times\left[\theta(E-\omega)\left(1+f_{\omega}-f_{q}\right)-\theta(\omega-E)\left(f_{\omega}+f_{q}\right)\right]
\end{aligned}
$$

The first $\omega$ range eq. 3.43 matches with the first theta function, whereas the second $\omega$ range eq. 3.44 matches with the second theta function.

With eq. 3.42, one obtains for the Dirac traces

$$
\begin{aligned}
& D_{T}(\omega, k, E, p, \mathbf{p k})=-\left(1-\frac{\omega^{2}}{k^{2}}\right)\left(k^{2}-\omega^{2}\right)\left[\left(E-\frac{\omega}{2}\right)^{2}-\frac{k^{2}}{4}\right]+ \\
& \quad+E \omega\left(1-\frac{\omega^{2}}{k^{2}}\right)\left(m_{\tilde{g}}^{2}-m_{\tilde{G}}^{2}\right)-\left(k^{2}-\omega^{2}\right)\left(m_{\tilde{g}} m_{\tilde{G}}+\frac{\omega^{2}}{k^{2}} \frac{m_{\tilde{g}}^{2}-m_{\tilde{G}}^{2}}{2}\right)-\left(1+\frac{\omega^{2}}{k^{2}}\right) \frac{\left(m_{\tilde{g}}^{2}-m_{\tilde{G}}^{2}\right)^{2}}{4} \\
& D_{L}(\omega, k, E, p, \mathbf{p k})=-\left(k^{2}-\omega^{2}\right)\left(E-\frac{\omega}{2}\right)^{2}+ \\
& \quad+k^{2} \frac{\left(m_{\tilde{g}}+m_{\tilde{G}}\right)^{2}}{4}+\omega\left(E-\frac{\omega}{2}\right)\left(m_{\tilde{g}}^{2}-m_{\tilde{G}}^{2}\right)+\frac{\left(m_{\tilde{g}}^{2}-m_{\tilde{G}}^{2}\right)^{2}}{4}
\end{aligned}
$$

Up to this point, the calculation is exact. Now I will start making approximations. The high temperature limit condition

$$
E \approx p \sim T \gg m_{\tilde{G}}, m_{\tilde{g}}
$$

will be used to simplify the Dirac traces eq. 3.46 .

As for the gluon spectral densities eq. 3.34, I will neglect the delta function parts and show in appendix D.1 that they do not contribute to the leading order terms of the result. The remaining parts of $\rho_{T / L}(\omega, k)$ have support only in the region

$$
-k \leq \omega \leq k
$$


because of the theta functions $\theta(|x|-1)$.

The integration over the loop three momentum $k$ is subdivided into a soft part for $k=$ $0 \ldots \bar{k}$ and a hard part for $k=\bar{k} \ldots \infty$, where $\bar{k} \sim \sqrt{g} T$.

\subsubsection{Soft loop momenta}

For soft momenta $k$, we have $E \gg k,|\omega|$. Therefore only the first $\omega$ integration range eq. 3.43 contributes, which I approximate to $\omega \approx-k \ldots k$. This is justified in appendix D.2; there one has to assume that $m_{\tilde{g}} \ll m_{g}$. I approximate $\left(1+f_{\omega}-f_{q}\right) \approx T / \omega$. Only the leading terms of the Dirac traces eq. 3.46 are kept:

$$
\begin{aligned}
& D_{L} \approx-E^{2}\left(k^{2}-\omega^{2}\right)=-E^{2} m_{g}^{2} z\left(1-x^{2}\right), \\
& D_{T} \approx-E^{2}\left(k^{2}-\omega^{2}\right)\left(1-\frac{\omega^{2}}{k^{2}}\right)=\left(1-x^{2}\right) D_{L} .
\end{aligned}
$$

Again, $x=\omega / k$ and $z=k^{2} / m_{g}^{2}$. This gives a contribution to the gravitino regeneration rate from soft gluons

$$
\begin{aligned}
\Gamma_{\tilde{G}}^{\text {ooft }} & =-\left.\frac{\Im(S)}{E}\right|_{k<\bar{k}}= \\
& =\frac{3}{64 \pi} C T m_{g}^{2} \int_{0}^{\frac{1}{\varepsilon}} d z \int_{-1}^{1} d x\left(1-x^{2}\right)\left[\frac{z}{A_{T}^{2}+\left(z+B_{T}\right)^{2}}+\frac{2 z}{A_{L}^{2}+\left(z+B_{L}\right)^{2}}\right]
\end{aligned}
$$

I have introduced the quantity $\varepsilon:=m_{g}^{2} / \bar{k}^{2} \sim g$ which is small in the weak coupling limit. Doing the $z$ integration first,

$$
\int_{0}^{\frac{1}{\varepsilon}} d z \frac{z}{A^{2}+(z+B)^{2}}=\ln \frac{1}{\varepsilon}+\frac{1}{2} \ln \frac{(1+\varepsilon B)^{2}+\varepsilon^{2} A^{2}}{A^{2}+B^{2}}-\frac{B}{A} \arctan \frac{A}{B+\varepsilon\left(A^{2}+B^{2}\right)}
$$

(this is also valid when the denominator of the integrand goes through zero, as can be seen by taking the limit after the integration) we find the leading logarithmic term analytically and a constant term which has to be determined numerically. Up to the factor of $\frac{3}{64 \pi} C T m_{g}^{2}$, the contribution to $\Gamma_{\tilde{G}}^{\text {oft }}$ from transverse gluons is $\frac{4}{3} \ln \frac{1}{\varepsilon}-0.374$ and the contribution from longitudinal gluons is $\frac{8}{3} \ln \frac{1}{\varepsilon}-5.214$.

The gravitino regeneration rate from soft gluons is

$$
\Gamma_{\tilde{G}}^{\mathrm{oft}}=\frac{N^{2}-1}{4 \pi} \frac{m_{\tilde{g}}^{2}}{M^{2} m_{\tilde{G}}^{2}} m_{g}^{2} T\left(\ln \frac{\bar{k}^{2}}{m_{g}^{2}}-1.397+\ldots\right) .
$$

Compared to the result for $\Gamma_{\tilde{G}}^{\mathrm{s} f t}$ obtained in [13], eq. (39), my coefficient of the logarithmic term is larger by a factor of $4\left(N^{2}-1\right)$. It seems that in [13] a gravitino-gluino-gluon vertex 
was used which is too small by a factor of 2 , and the summation over the gluonic colour degrees of freedom has been forgotten. Up to the numerical prefactor, eq. (37) of [13] (which differs from their eq. (39) by a factor of 2) suggests agreement in the constant term.

\subsubsection{Hard loop momenta}

For hard loop three momenta $k>\bar{k}$ we have to keep also terms $\mathcal{O}\left(\omega^{4}, \omega^{2} k^{2}, k^{4}\right)$ in the Dirac traces eq. 3.46, but we can still neglect terms containing mass factors:

$$
\begin{aligned}
& D_{T} \approx-\frac{m_{g}^{4}}{\delta^{2}} z\left(1-x^{2}\right)^{2}\left[\left(1-\frac{\delta}{2} x \sqrt{z}\right)^{2}-\frac{\delta^{2}}{4} z\right], \\
& D_{L} \approx-\frac{m_{g}^{4}}{\delta^{2}} z\left(1-x^{2}\right)\left(1-\frac{\delta}{2} x \sqrt{z}\right)^{2} .
\end{aligned}
$$

Here I introduced a new small quantity $\delta:=m_{g} / E \sim g$. For the gluon spectral densities eq. 3.34 I use the approximations

$$
\rho_{T}(\omega, k) \approx \frac{3}{4} m_{g}^{-2} \frac{x /\left(1-x^{2}\right)}{z^{2}} \theta(|x|-1), \quad \rho_{L}(\omega, k) \approx \frac{3}{4} m_{g}^{-2} \frac{2 x}{z^{2}} \theta(|x|-1) .
$$

Since $z>1 / \varepsilon$ for hard gluons, whereas $\left|A_{T / L}(x)\right|,\left|B_{T / L}(x)\right| \simeq \mathcal{O}(1)$ for most values of $x$, it is allowed to neglect the latter quantities in the denominators (this was also checked numerically).

Now it is convenient to subdivide the integration over $z$ into two parts: starting from $\Im(S)$, eq. 3.45, we have the contribution to the gravitino regeneration rate from hard gluons

$$
\begin{aligned}
\Gamma_{\tilde{G}}^{\mathrm{hard}}= & -\left.\frac{\Im(S)}{E}\right|_{k>\bar{k}}=\frac{3}{64 \pi} C m_{g}^{3} \int_{\frac{1}{\varepsilon}}^{\infty} d z \int d x \frac{x}{\sqrt{z}}\left(1-x^{2}\right)\left[3\left(1-\frac{\delta}{2} x \sqrt{z}\right)^{2}-\frac{\delta^{2}}{4} z\right] \times \\
& \times \theta(|x|-1)\left[\theta\left(\frac{1}{\delta \sqrt{z}}-x\right)\left(1+f_{\omega}-f_{q}\right)-\theta\left(x-\frac{1}{\delta \sqrt{z}}\right)\left(f_{\omega}+f_{q}\right)\right]= \\
= & \frac{3}{64 \pi} C m_{g}^{3}\left(I_{E}+I_{\infty}\right) .
\end{aligned}
$$

The $z$ integration region is divided into an intermediate range $z=1 / \varepsilon \ldots 1 / \delta^{2}$ (corresponding to $k=\bar{k} \ldots E$ ), yielding $I_{E}$, and a range of large $z, z>1 / \delta^{2}$, yielding $I_{\infty}$.

To both integrals $I_{E}$ and $I_{\infty}$ only the term proportional to $\left(1+f_{\omega}-f_{q}\right)$ contributes: for $k<E$ one is always in the first $\omega$ integration range eq. 3.43, corresponding to the first statistical factor. For $k>E$ the second $\omega$ integration range eq. 3.44, corresponding to the 
second statistical factor $\left(f_{\omega}+f_{q}\right)$, is $\omega=k \ldots k+2 E$ where the gluon spectral function has no support.

The leading logarithmic term of $\Gamma_{\tilde{G}}^{\text {hard }}$ can be obtained analytically from $I_{E}$ omitting constant and higher order terms in the development of the statistical factor

$$
\left(1+f_{\omega}-f_{q}\right)=\frac{T}{\omega}+\ldots
$$

In $I_{E}, x$ integration goes from -1 to 1 , yielding

$$
I_{E}=4 \frac{T}{m_{g}}\left[\ln \frac{E^{2}}{\bar{k}^{2}}-\frac{1}{30}+\mathcal{O}(1)\right] .
$$

Note that the logarithm containing the arbitrary scale $\bar{k}$ matches the logarithm of the rate for soft gluons eq. 3.52: the end result is independent of $\bar{k}$, as it should be.

Unfortunately the expression in square brackets in eq. 3.57 receives also $\mathcal{O}(1)$ contributions from the $\mathcal{O}(1)$ terms in the development of the statistical factor eq. 3.56. It seems impossible to isolate the constant term of $\Gamma_{\tilde{G}}^{\text {hard }}$ analytically. On the other hand, if one does the integration numerically one has to give the coupling $g$ a specific numerical value, and contributions from different orders in $g$ get mixed up, as already pointed out in [29]. In practice however, assuming that the perturbation series behaves reasonably, one can numerically isolate the constant term of $I_{E}$ by choosing $g$ small enough. Small enough means that the result, after subtraction of the leading logarithmic term $\propto \ln \frac{E^{2}}{k^{2}}$, exhibits no dependence on $g$ any more.

The constant $K_{E}(E)$,

$$
I_{E}=4 \frac{T}{m_{g}}\left[\ln \frac{E^{2}}{\bar{k}^{2}}+K_{E}(E)+\ldots\right],
$$

is given by the weak coupling limit of

$$
K_{E}(E)=\frac{m_{g}}{4 T}\left\{\int_{\frac{1}{\varepsilon}}^{1 / \delta^{2}} d z \int_{-1}^{1} d x \frac{x}{\sqrt{z}}\left(1-x^{2}\right)\left[3\left(1-\frac{\delta}{2} x \sqrt{z}\right)^{2}-\frac{\delta^{2}}{4} z\right]\left(1+f_{\omega}-f_{q}\right)\right\}-\ln \frac{\varepsilon}{\delta^{2}} .
$$

Using the stochastic integration program VEGAS and setting $\varepsilon \equiv \frac{m_{g}^{2}}{\bar{k}^{2}}=\tilde{g}$ with $\tilde{g}:=\frac{m_{g}}{T}=$ $g \sqrt{\frac{N+n_{f}}{6}}$, I have evaluated eq. 3.59 for different values of $E$. Table 3.1 shows the results ( $\tilde{g}$ was set to $\left.10^{-6}\right)$.

As for $I_{\infty}$, one can use the approximation eq. 3.56 for small $\omega<E$ and

$$
\begin{aligned}
f_{B}(-\omega) & \approx \exp \left(\frac{\omega}{T}\right), \\
f_{F}(E-\omega) & \approx \exp \left(\frac{\omega-E}{T}\right)
\end{aligned}
$$




\begin{tabular}{|c|c|c|}
\hline $\begin{array}{l}\text { Gravitino en- } \\
\text { ergy } E / T\end{array}$ & $K_{E}(E)$ & $K_{\infty}(E)$ \\
\hline $10^{-2}$ & -0.033 & 6.5 \\
\hline $3 \cdot 10^{-2}$ & -0.033 & 5.4 \\
\hline $10^{-1}$ & -0.034 & 4.1 \\
\hline $3 \cdot 10^{-1}$ & -0.041 & 3.0 \\
\hline 1 & -0.10 & 1.6 \\
\hline 3 & -0.23 & 0.80 \\
\hline $10^{1}$ & 0.29 & 0.78 \\
\hline $3 \cdot 10^{1}$ & 3.9 & 1.8 \\
\hline $10^{2}$ & 21 & 5.9 \\
\hline $10^{3}$ & 260 & 58 \\
\hline $10^{4}$ & 2700 & 580 \\
\hline
\end{tabular}

Table 3.1: The leading order constant terms in $\Gamma_{\tilde{G}}^{\text {hard }}$ for different values of the gravitino energy E. $K_{E}(E)$, as defined in eq. 3.59, gives the contribution from intermediate loop momenta $\bar{k}<k<E . K_{\infty}(E)$, as defined in eq. 3.62, gives the contribution from large loop momenta $k>E$.

for large $\omega>E$ in order to estimate the order of the result for $E \sim T$ :

$$
I_{\infty}=4 \frac{T}{m_{g}}\left[\frac{6}{5}+e^{-E / T}\left(1+e^{-E / T}\right)+\mathcal{O}(1)\right]=4 \frac{T}{m_{g}} K_{\infty}(E) .
$$

This consideration shows in particular that there is no further logarithmic contribution to $\Gamma_{\tilde{G}}^{\text {hard }}$ from $I_{\infty}$, but only a constant one. However, since the approximations eqs. 3.56 and 3.60 are not good enough, the exact value can only be obtained from a numerical integration, just as with the constant contained in $I_{E}$.

$K_{\infty}(E)$ as introduced in eq. 3.61 is given by

$$
K_{\infty}(E)=\frac{m_{g}}{4 T} \int_{1 / \delta^{2}}^{\infty} d z \int_{-1}^{-1+\frac{2}{\delta \sqrt{z}}} d x \frac{x}{\sqrt{z}}\left(1-x^{2}\right)\left[3\left(1-\frac{\delta}{2} x \sqrt{z}\right)^{2}-\frac{\delta^{2}}{4} z\right]\left(1+f_{\omega}-f_{q}\right) .
$$


Numerical values of $K_{\infty}(E)$ for different gravitino energies $E$ are given in table 3.1. Again, $\tilde{g}$ was set to $10^{-6}$, and I used $\left(10 \frac{E^{2}}{T^{2}}+10^{4}\right) / \tilde{g}^{2}$ instead of infinity as the upper limit of the $z$ integration.

Both constants $K_{E}(E)$ and $K_{\infty}(E)$ are small for values $E<T$ but get large for values $E \gg T$ : the $\mathcal{O}(1)$ terms omitted in the approximation eq. 3.56 lead to terms linear in $E / T$ in the constants $K_{E}(E)$ and $K_{\infty}(E)$.

The gravitino regeneration rate from hard gluons is

$$
\Gamma_{\tilde{G}}^{\text {hard }}=\frac{N^{2}-1}{4 \pi} \frac{m_{\tilde{g}}^{2}}{M^{2} m_{\tilde{G}}^{2}} m_{g}^{2} T\left[\ln \frac{T^{2}}{\bar{k}^{2}}+2 \ln \frac{E}{T}+K_{E}(E)+K_{\infty}(E)+\ldots\right] .
$$

The dots represent contributions of higher order in the coupling $g$. The leading logarithmic term stems from $I_{E}$ which represents the contribution from the intermediate gluon energy range $k=\bar{k} \ldots E$.

Here a remark is in order as to why one cannot use a non-resummed gluon progagator when calculating $\Gamma_{\tilde{G}}^{\mathrm{hard}}$, which would be okay following the rules of HTL effective perturbation theory. Using a bare gluon progagator for the calculation of the hard gluon contribution leads to a gravitino regeneration rate of the order of

$$
\Gamma_{\tilde{G}}^{\mathrm{bare}} \sim \frac{m_{\tilde{g}}^{6}}{M^{2} m_{\tilde{G}}^{2} T} \ln \frac{T^{2}}{\bar{k}^{2}},
$$

a result completely different from eq. 3.63. This is, on one hand, no surprise. There is a clear correspondence between a Boltzmann collision term obtained from a $1 \rightarrow 2$ decay and the one obtained from a pure one loop self energy diagram (see section 3.1). One expects, and it can be shown explicitly, that the resulting imaginary part of the self energy contains the matrix element for gluino decay into a gravitino and a gluino which is $\sim \frac{m_{\tilde{g}}^{6}}{M^{2} m_{\tilde{G}}^{2}}$, and for dimensional reasons one must obtain the regeneration rate eq. 3.64. On the other hand, the results for a bare and for a resummed gluon propagator should have the same high-energy behavior which here is obviously not the case.

An attempt of an explanation for this surprising effect is that the thermal mass of the gluon plays a crucial role in gravitino production and disappearance processes, not only for soft gluons, but for gluons of energy up to $\sim T$ : the whole gravitino regeneration rate $\Gamma_{\tilde{G}}$ is proportional to the thermal gluon mass squared. Since such a mass is not present when one uses the bare gluon propagator, such a calculation does not give the leading result, which here comes from a subset of all two-and-higher-loop gravitino self energy diagrams.

The total result for the gravitino regeneration rate $\Gamma_{\tilde{G}}$ reads

$$
\Gamma_{\tilde{G}}=\Gamma_{\tilde{G}}^{\text {soft }}+\Gamma_{\tilde{G}}^{\text {hard }}=
$$




$$
\frac{N^{2}-1}{4 \pi} \frac{m_{\tilde{g}}^{2}}{M^{2} m_{\tilde{G}}^{2}} m_{g}^{2} T\left[\ln \frac{T^{2}}{m_{g}^{2}}+2 \ln \frac{E}{T}-1.397+K_{E}(E)+K_{\infty}(E)+\ldots\right]
$$

It is given here including the leading logarithmic and the corresponding constant term. Note that the constant term has a non-negligible dependence on the gravitino energy. The energy dependence beyond the logarithmic one can only be obtained from a calculation in the framework of thermal field theory. Previous estimates of the constant $[10,11,14]$ were based on zero temperature calculations of the production processes, therefore they could not yield the contributions $K_{E}(E)$ and $K_{\infty}(E)$ to $\Gamma_{\tilde{G}}$.

The term $2 \ln \frac{E}{T}$ of the expression in square brackets in eq. 3.65 makes the constant negative for small gravitino energies $E$. It is balanced by the leading logarithmic term $\ln \frac{T^{2}}{m_{g}^{2}} \sim \ln \frac{1}{g^{2}}$ such that $\Gamma_{\tilde{G}}$ remains positive as long as the gravitino energy does not become soft, $E \gg g T$. For soft gravitino energies, one would have had to use also effective vertices when calculating the gravitino self energy diagram. Therefore it is no surprise that $\Gamma_{\tilde{G}}$ turns negative for soft gravitino energies.

Fig. 3.4 shows the gravitino regeneration rate up to the order given in eq. 3.65 as a function

of the gravitino energy $E$ (I used $N=3$ and $n_{f}=6$ for $m_{g}^{2}$, eq. 3.28, and the running coupling $\left.g\left(T=10^{10} \mathrm{GeV}\right)=0.85\right)$. With decreasing $E$ the regeneration rate $\Gamma_{\tilde{G}}(E)$ as calculated here turns from positive to negative values in the region $E \approx T$. Because of the large value of $g$, the unphysical behavior of $\Gamma_{\tilde{G}}$ for soft gravitino energy starts already at $E \approx T$.

\subsubsection{The Boltzmann collision term}

Using eq. 3.20 with $f_{\tilde{G}}=0$ we find for the Boltzmann collision term $\mathcal{C}_{\Sigma}$ obtained from the self energy method

$$
\mathcal{C}_{\Sigma}=\frac{3 \zeta(3)}{16 \pi^{3}}\left(N^{2}-1\right) \frac{m_{\tilde{g}}^{2}}{M^{2} m_{\tilde{G}}^{2}} m_{g}^{2} T^{4}\left(\ln \frac{T^{2}}{m_{g}^{2}}+1.366+\ldots\right)
$$

The constant of 1.366 after the logarithm is made up of four contributions: a contribution of 1.978 from the term $2 \ln \frac{E}{T}$ in eq. 3.65, the contribution of -1.397 from $\Gamma_{\tilde{G}}^{\mathrm{soft}}$, a contribution of -0.177 from $K_{E}(E)$, and a contribution of 0.962 from $K_{\infty}(E)$. The last two contributions have been obtained by numerical integration over $E$.

Substituting the expression eq. 3.28 for $m_{g}^{2}$ yields

$$
\mathcal{C}_{\Sigma}=\frac{\zeta(3)}{32 \pi^{3}}\left(N^{2}-1\right)\left(N+n_{f}\right) \frac{m_{\tilde{g}}^{2}}{M^{2} m_{\tilde{G}}^{2}} g^{2} T^{6}\left[\ln \frac{1}{g^{2}}-\ln \left(N+n_{f}\right)+3.158+\ldots\right]
$$




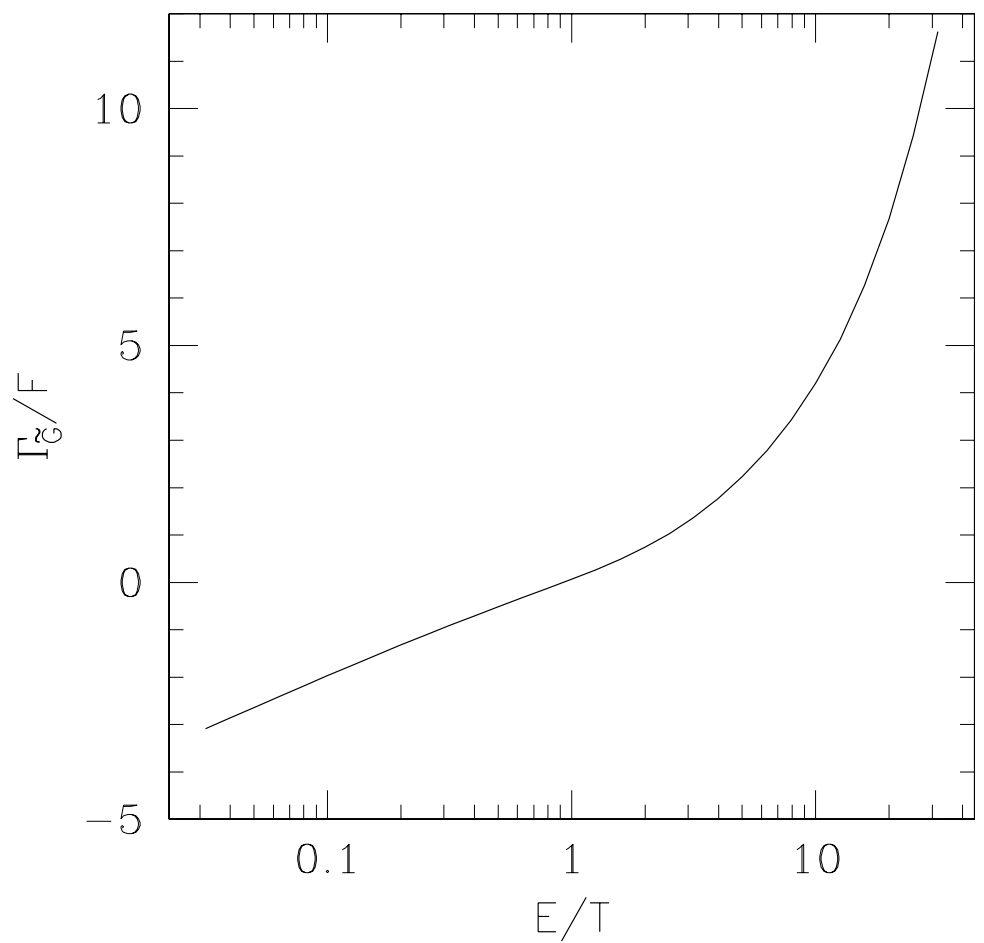

Figure 3.4: The gravitino regeneration rate $\Gamma_{\tilde{G}}(E)$ as given in eq. 3.65 up to a factor of $F=\frac{N^{2}-1}{4 \pi} \frac{m_{\tilde{g}}^{2}}{M^{2} m_{\tilde{G}}^{2}} m_{g}^{2} T$.

The coefficient of the leading logarithmic term of $\mathcal{C}_{\Sigma}$ is analytically the same as the coefficient of the leading logarithmic term of $\mathcal{C}_{2 \rightarrow 2}$, eq. 2.31 , in the limit of small gravitino mass. The constant in the Boltzmann collision term however can only be obtained consistently from the self energy calculation, and not from starting from the $2 \rightarrow 2$ gravitino production processes. 


\section{Chapter 4}

\section{Baryon Asymmetry And Dark Matter}

The result eq. 3.67 for the Boltzmann collision term for gravitino production contains two improvements compared to older results [11, 13, 14]. First, the constant term has been calculated for the first time consistently in a finite temperature approach. Second, the coefficient of the leading logarithmic term has been determined with improved accuracy. The result eq. 2.31 for this coefficient obtained from considering $2 \rightarrow 2$ gravitino production processes and the result eq. 3.67 obtained via the imaginary part of the gravitino self energy coincide analytically.

In view of these improvements it seems worthwhile to update the analysis we have presented in [14], where the implications of a large baryogenesis temperature $T_{B}=$ $\mathcal{O}\left(10^{10} \mathrm{GeV}\right)$ on the mass spectrum of superparticles in supersymmetric extensions of the standard model have been studied. It turns out that the phenomenological results remain essentially unchanged. The new gravitino constraints are slightly relaxed compared to the old ones, because the gravitino production cross section we used in [14] was about $33 \%$ larger than the new one.

This chapter is organized as follows. First, a motivation is given why one is interested in a large baryogenesis temperature $T_{B}=\mathcal{O}\left(10^{10} \mathrm{GeV}\right)$, and the problems due to the presence of gravitinos in such a scenario are explained. Then the new formulae for the gravitino abundance and contribution to $\Omega h^{2}$ are derived. They predict values around $25 \%$ smaller than the values given by the old formulae of [14]. Finally a scenario with a baryogenesis temperature $T_{B}=\mathcal{O}\left(10^{10} \mathrm{GeV}\right)$ is presented which satisfies all constraints due to the existence of gravitinos. 


\subsection{Baryogenesis and the gravitino problem}

A possible answer to the question why there is more matter than antimatter in the universe is baryogenesis through leptogenesis [9]. In the leptogenesis model, the creation of the cosmological baryon asymmetry is accounted for by the following mechanism: one starts from a non-vanishing lepton asymmetry $L$ that is transformed into a non-zero baryon asymmetry $B$ via lepton-number violating sphaleron processes [33]. $L$ can be created from out-of-equilibrium decay of heavy Majorana neutrinos $N$. Assuming a similar mass spectrum in the quark and lepton sector, one finds a natural mass scale of the lightest heavy neutrino $N_{1}$ in the order of $10^{10} \mathrm{GeV} . N_{1}$ get out of thermal equilibrium at $T \simeq m_{N_{1}}$. Therefore the temperature scale of baryogenesis in such a model is $T_{B} \simeq \mathcal{O}\left(10^{10} \mathrm{GeV}\right)$ [34]. Assuming inflation, this means that we need a reheating temperature at least as large as $T_{R} \simeq \mathcal{O}\left(10^{10} \mathrm{GeV}\right)$. The question arises whether such a reheating temperature is consistent with constraints due to the existence of gravitinos.

To find a viable cosmological scenario one has to avoid two types of gravitino problems $[8,10,11,35]$ : First, the mass of all gravitinos in the universe must not exceed the closure limit, $\Omega_{\tilde{G}} \equiv \frac{\rho_{\tilde{G}}}{\rho_{c}}<1\left(\rho_{c}=3 H_{0}^{2} M^{2}=1.05 h^{2} 10^{-5} \mathrm{GeV} \mathrm{cm}^{-3}\right.$ is the critical energy density $)$, and second, decay products of unstable gravitinos or of particles decaying into gravitinos must not alter the observed abundances of light elements in the universe. The latter constraint is called big bang nucleosynthesis (BBN) constraint. Both gravitino problems get more serious as $T_{R}$ increases, because the gravitino abundance is proportional to $T_{R}$. It was widely believed that it is difficult to have $T_{R} \geq \mathcal{O}\left(10^{9}\right) \mathrm{GeV}$.

In [14] we showed that it is possible to have $T_{R} \geq \mathcal{O}\left(10^{10}\right) \mathrm{GeV}$ if certain conditions on the mass spectrum of supersymmetric particles are satisfied: in our scenario, the gravitino is the lightest supersymmetric particle (LSP) with a mass between $10 \mathrm{GeV}$ and $100 \mathrm{GeV}$, followed by a higgsino-like neutralino as next-to-lightest supersymmetric particle (NSP).

\subsection{The gravitino abundance after inflation}

From the Boltzmann equation 2.20 one obtains for the gravitino abundance at temperatures $T<T_{B}$, assuming constant entropy,

$$
Y_{\tilde{G}}(T):=\frac{n_{\tilde{G}}(T)}{n_{\mathrm{rad}}(T)} \approx \frac{g_{\star S}(T)}{g_{\star S}\left(T_{B}\right)} \frac{\mathcal{C}_{\tilde{G}}\left(T_{B}\right)}{H\left(T_{B}\right) n_{\mathrm{rad}}\left(T_{B}\right)},
$$

where $g_{\star S}(T)$ is the number of effectively massless degrees of freedom [25]. For $T<1 \mathrm{MeV}$, i.e. after nucleosynthesis, $g_{\star S}(T)=2+\frac{21}{4}\left(\frac{T_{\nu}}{T}\right)^{3}=\frac{43}{11}$, and $g_{\star S}\left(T_{B}\right)=\frac{915}{4}$ in the MSSM. 
With

$$
H(T)=\sqrt{\frac{g_{\star}(T)}{90}} \frac{\pi}{M} T^{2}
$$

(where $g_{\star}(T)=g_{\star S}(T)$ for $T$ as high as $\sim \mathcal{O}\left(T_{B}\right)$ ) one obtains for light gravitinos $\left(m_{\tilde{G}} \ll\right.$ $m_{\tilde{g}}(\mu), \mu \simeq 100 \mathrm{GeV}$ ) from eqs. (4.1) and (3.67) for the gravitino abundance and the contribution to $\Omega h^{2}$,

$$
\begin{gathered}
Y_{\tilde{G}}=2.26 \cdot 10^{-10}\left(\frac{T_{B}}{10^{10} \mathrm{GeV}}\right)\left(\frac{100 \mathrm{GeV}}{m_{\tilde{G}}}\right)^{2}\left(\frac{m_{\tilde{g}}(\mu)}{1 \mathrm{TeV}}\right)^{2}, \\
\Omega_{\tilde{G}} h^{2}=m_{\tilde{G}} Y_{\tilde{G}}(T) n_{\mathrm{rad}}(T) h^{2} \rho_{c}^{-1} \\
=0.43\left(\frac{T_{B}}{10^{10} \mathrm{GeV}}\right)\left(\frac{100 \mathrm{GeV}}{m_{\tilde{G}}}\right)\left(\frac{m_{\tilde{g}}(\mu)}{1 \mathrm{TeV}}\right)^{2} .
\end{gathered}
$$

Here I have used $g\left(T_{B}\right)=0.85, n_{\mathrm{rad}}\left(T_{0}=2.7 \mathrm{~K}\right)=200 \mathrm{~cm}^{-3}$, and $m_{\tilde{g}}(T)=\frac{g^{2}(T)}{g^{2}(\mu)} m_{\tilde{g}}(\mu)$. Note that the temperature dependence of the gluino mass is only the logarithmic one from the running coupling $g(T)$. Using a thermal gluino mass $m_{\tilde{g}} \sim T$ would not be appropriate here, since $m_{\tilde{g}}$ in the Boltzmann collision term $\mathcal{C}_{\Sigma}$, eq. 3.67, represents the scale of supersymmetry breaking rather than an effective particle mass at high temperature.

Eqs. 4.3 and 4.4 predict a gravitino abundance and a gravitino contribution to $\Omega h^{2}$ which is about three quarters of the one we have found in [14] (cf. eqs. (8) and (9) there).

Compare the thermally averaged cross section $C(T)$, eq. (6) of [14],

$$
C^{\text {old }}(T) \simeq 10 \frac{g^{2}(T)}{M^{2}}\left(1+\frac{m_{\tilde{g}}^{2}(T)}{3 m_{\tilde{G}}^{2}}\right)\left(\ln \frac{1}{g^{2}(T)}+2.7\right)
$$

with the new value

$$
C^{\text {new }}(T) \simeq 17.6 \frac{g^{2}(T)}{M^{2}} \frac{m_{\tilde{g}}^{2}(T)}{3 m_{\tilde{G}}^{2}}\left(\ln \frac{1}{g^{2}(T)}+0.96\right):
$$

in the limit of a small gravitino mass, the new value is about three quarters of the old value. The new prefactor of 17.6 instead of 10 comes from the factor of $\zeta(3)^{2}$ mentioned after eq. 2.30 times the correction due to the quantum statistical factor which is included both in $\mathcal{C}_{2 \rightarrow 2}$ (eq. 2.31) and in $\mathcal{C}_{\Sigma}$ (eq. 3.67). In obtaining the old constant (the term proportional to 2.7 in eq. 4.5 ) we had neglected several contributions which should contribute to the same order, thus overestimating the constant term. 


\subsection{An inflationary scenario with $T_{R} \simeq T_{B} \simeq$ $\mathcal{O}\left(10^{10} \mathrm{GeV}\right)$}

In the case of the $2 \rightarrow 2$ gravitino production cross sections (section 2.2), using the full theory rather than the effective theory for light gravitinos completes the factor $\frac{m_{\tilde{g}}^{2}}{3 m_{\tilde{G}}^{2}}$ to give the sum $\left(1+\frac{m_{\tilde{g}}^{2}}{3 m_{\tilde{G}}^{2}}\right)$. Under the assumption that this is valid not only for the coefficient of the leading logarithmic term, as it has been shown in eq. 2.31, but also for the corresponding constant term, the phenomenological conclusions of [14] remain essentially unchanged. In this section, they are summarized again.

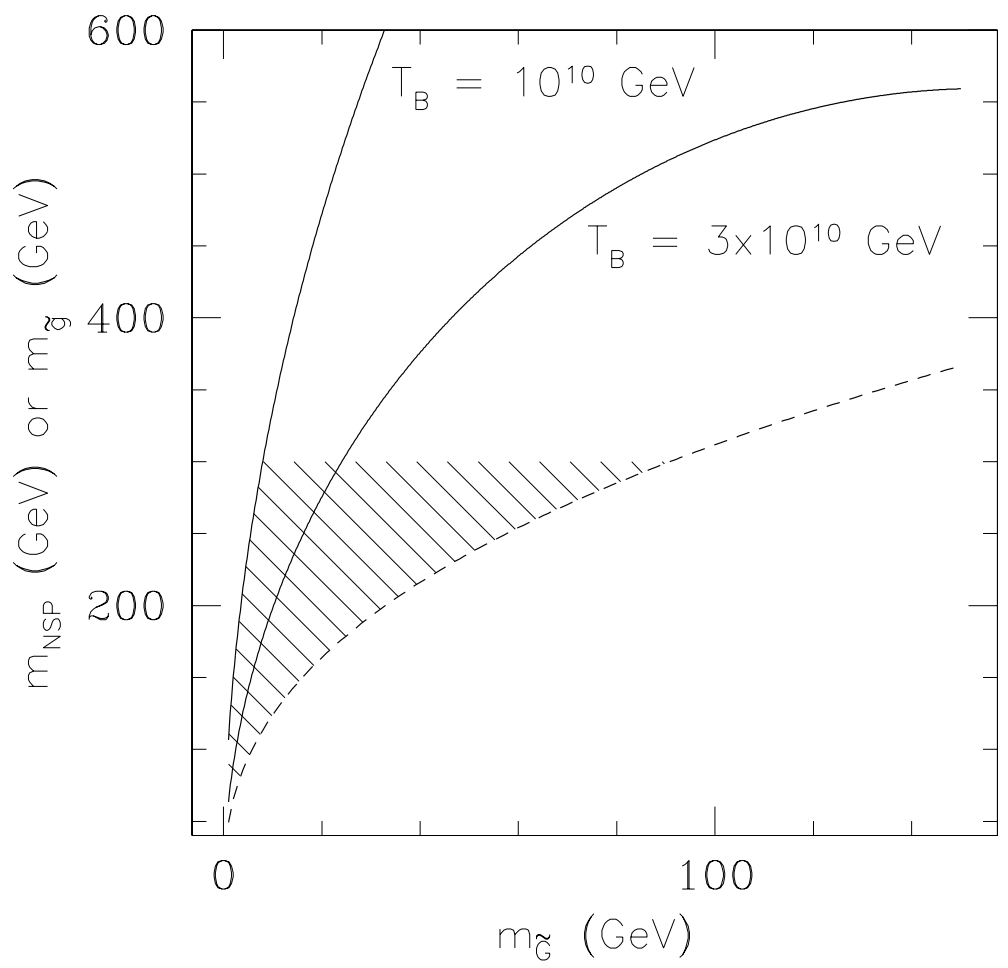

Figure 4.1: Upper and lower bounds on the NSP mass as function of the gravitino mass. The full lines represent the upper bound on the gluino mass $m_{\tilde{g}}>m_{N S P}$ for different reheating temperatures. The dashed line is the lower bound on $m_{N S P}$ which follows from the NSP lifetime. A higgsino-like NSP with a mass in the shaded area satisfies all cosmological constraints including those from primordial nucleosynthesis.

First, consider the constraint from the closure limit: From the condition $\Omega_{\tilde{G}}=$ $Y_{\tilde{G}} m_{\tilde{G}} \frac{n_{\mathrm{rad}}}{\rho_{c}} \leq 1$ follows an allowed region in the $m_{\tilde{G}}-m_{\tilde{g}}$ plane which is shown in fig. 4.1 
for two different values of $T_{R}$ ( $h$ was set to $0.7[36]$ ). The allowed regions are below the solid lines, respectively. Compared to the corresponding fig. 1 in our previous analysis in [14], the allowed maximum gluino mass as a function of the gravitino mass has shifted towards smaller values, and a reheating temperature as large as $T_{R} \simeq \mathcal{O}\left(10^{11} \mathrm{GeV}\right)$ seems completely impossible now. The effect of the smaller gravitino abundance has been overcompensated by the smaller Hubble parameter $h=0.7$ (in [14] we have used $h=1$ ).

Second, concerning the BBN constraint, consider a nonrelativistic particle $X$ decaying into electrically and strongly interacting relativistic particles with a lifetime $\tau_{X}$. Roughly speaking, the decay changes the abundances of light elements the more severely the longer $\tau_{X}$ and the higher the energy density $m_{X} Y_{X}$ are. These constraints have been studied in detail by several groups $[11,35]$.

From fig. 3 in [35] one reads off that the following conditions are sufficient in order not to come into conflict with the measured abundance data:

$$
\left\{\begin{array}{l}
\tau_{X}<2 \cdot 10^{6} \mathrm{~s}, \\
m_{X} Y_{X}<4 \cdot 10^{-10} \mathrm{GeV} \Leftrightarrow \Omega_{\mathrm{NSP}} h^{2}<0.008 .
\end{array}\right.
$$

One can easily exclude the case of an unstable gravitino [14]. Let us limit ourselves to the case where the gravitino is the LSP. The NSP then plays the role of the particle X. The lifetime of a fermion decaying into its scalar partner and a gravitino is

$$
\tau_{\mathrm{NSP}}=48 \pi \frac{m_{\tilde{G}}^{2} M^{2}}{m_{\mathrm{NSP}}^{5}} .
$$

From this we obtain an allowed region in the $m_{\tilde{G}}-m_{\text {NSP }}$ plane. In fig. 4.1 , it is the region above the dashed line.

The best studied NSP candidate is the neutralino. The neutralino $\chi$ is a mixture of the photino, the wino and the two neutral higgsinos:

$$
\chi=N_{1} \tilde{b}+N_{2} \tilde{W}_{3}+N_{3} \tilde{h}_{1}^{0}+N_{4} \tilde{h}_{2}^{0} .
$$

Depending on its composition, one can talk of gaugino-like or higgsino-like neutralinos. Edsjö and Gondolo [37] studied $\Omega_{\chi} h^{2}$ as a function of neutralino mass $m_{\chi}$ and composition $Z_{g}=\left|N_{1}\right|^{2}+\left|N_{2}\right|^{2}$ in a large SUSY parameter range. They found that for a broad range of $m_{\chi}$ and $Z_{g}, \Omega_{\chi} h^{2}>0.025$, thus a stable neutralino would be a good dark matter candidate in this range. Fig. 4.2 (taken from [37]) shows the distribution of such models. However for a higgsino-like neutralino, i.e. $Z_{g}<0.5$, there is also a broad range of $m_{\chi}$ for which $\Omega_{\chi} h^{2}<0.025$. If one decreases the limit to the value we need, $\Omega_{\chi} h^{2}<0.008$, the allowed neutralino mass range only slightly decreases to $80 \mathrm{GeV}<m_{\chi}<300 \mathrm{GeV}$ [38]. 


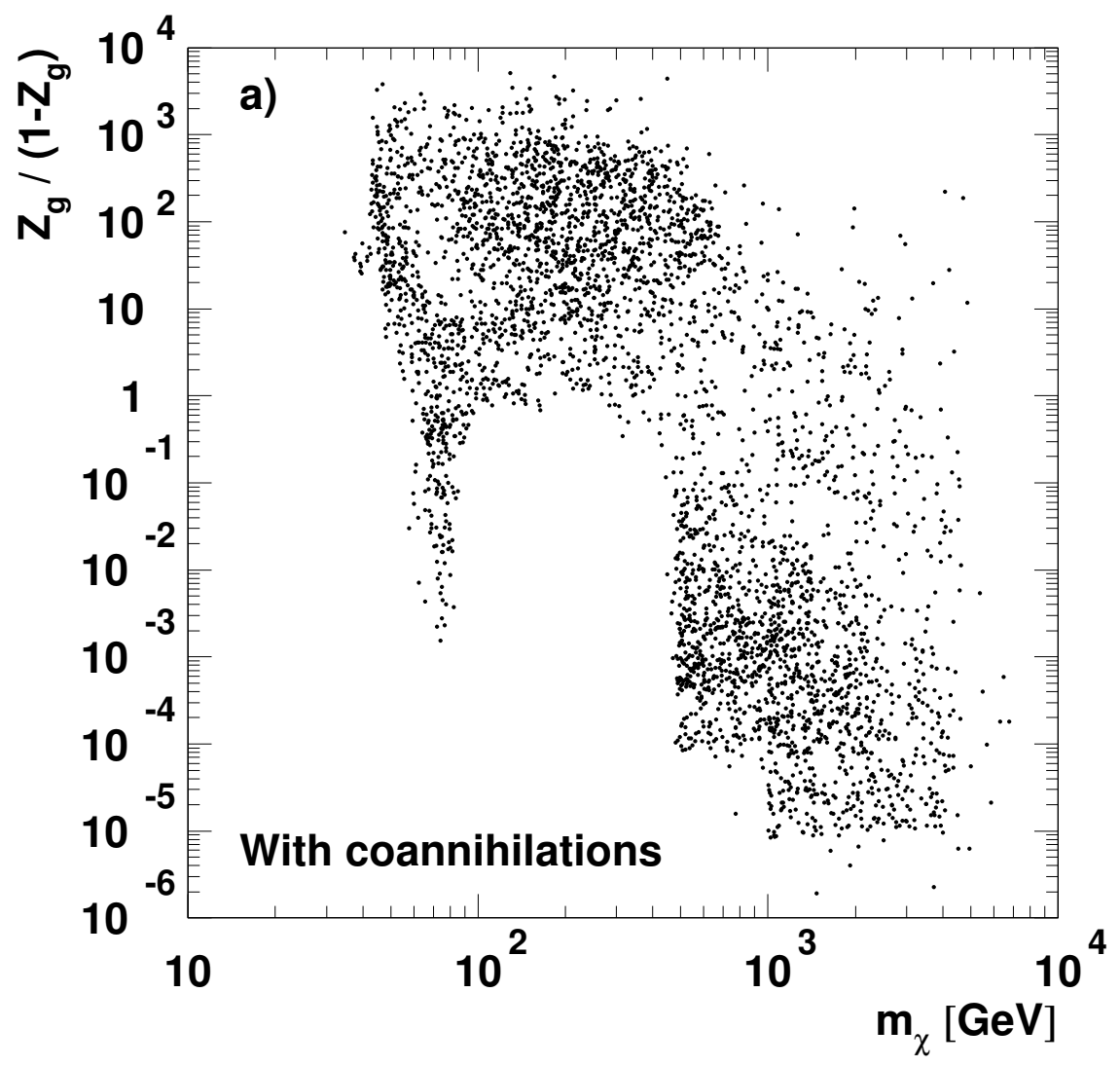

Figure 4.2: Neutralino masses $m_{\chi}$ and compositions $Z_{g} /\left(1-Z_{g}\right)$ for models with $\Omega_{\chi} h^{2}>$ 0.025 , including coannihilations between neutralinos and charginos (figure taken from [37]).

The shaded region in fig. 4.1 shows the $m_{\tilde{G}}-m_{\text {NSP }}$ range where all constraints are satisfied for a reheating temperature $T_{R}=10^{10} \mathrm{GeV}$. An example for a possible SUSY mass spectrum would be: a gravitino as LSP, $m_{\tilde{G}} \sim 50 \mathrm{GeV}$, followed by a higgsino-like neutralino as NSP, $m_{\chi} \sim 250 \mathrm{GeV}$, and a gluino mass $m_{\tilde{g}} \sim 500 \mathrm{GeV}$.

Note that $T_{R} \simeq T_{B} \simeq \mathcal{O}\left(10^{10} \mathrm{GeV}\right)$ leads to $\Omega_{\tilde{G}} h^{2}=0.1 \ldots 1$ in a consistent gravitino mass range. This is illustrated in fig. 4.3. Compared to the corresponding fig. 2 in our previous analysis in [14], $\Omega_{\tilde{G}} h^{2}$ as a function of the gravitino mass has become slightly smaller (cf. eq. 4.4). Yet with the new numbers, gravitinos may still be the dominant part of dark matter. 


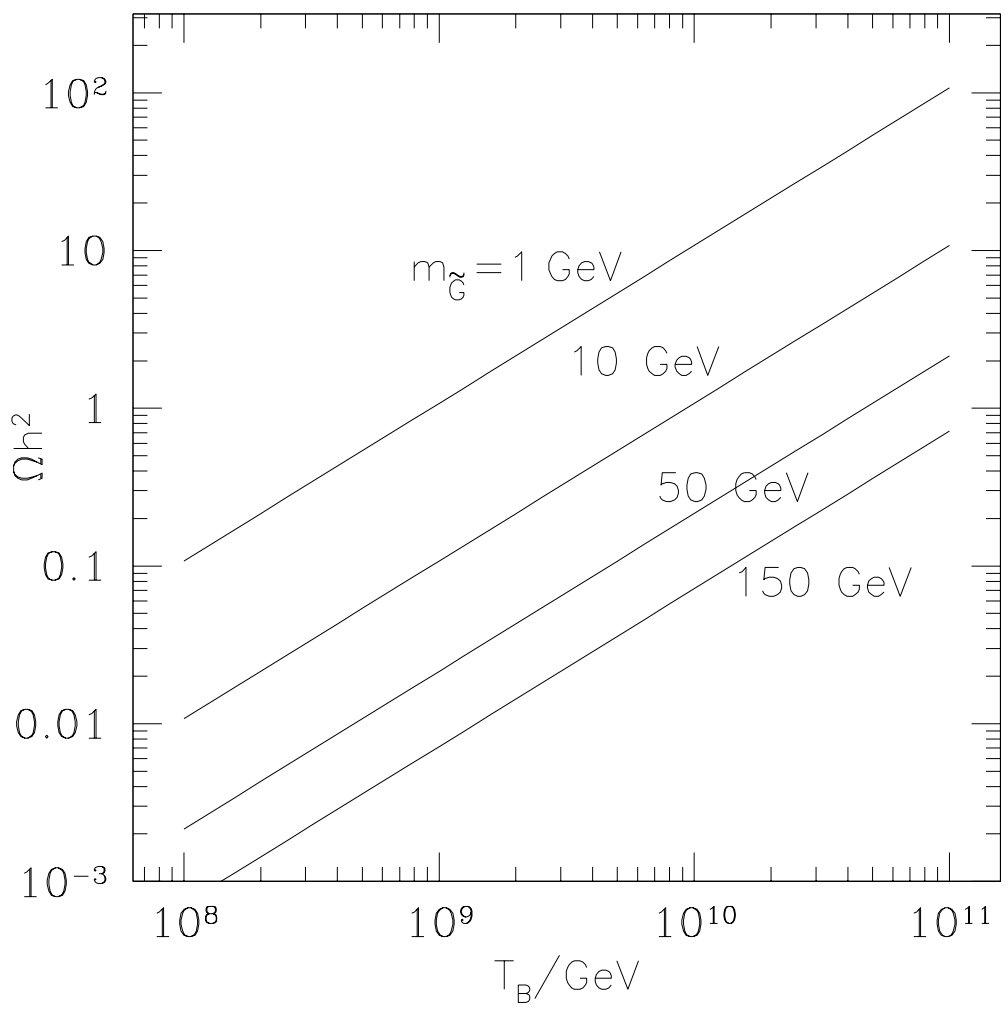

Figure 4.3: Contribution of gravitinos to the density parameter $\Omega_{\tilde{G}} h^{2}$ for different gravitino masses $m_{\tilde{G}}$ as function of the reheating temperature $T_{R}$. The gluino mass has been set to $m_{\tilde{g}}=500 \mathrm{GeV}$. 


\section{Conclusions}

In this thesis the Boltzmann collision term $\mathcal{C}_{\tilde{G}}$ governing the Boltzmann equation for the time evolution of the gravitino number density has been calculated to the leading logarithmic and corresponding constant order in the strong coupling. As an application, the gravitino constraints on a cosmological scenario with a large reheating temperature $T_{R}$ have been re-analyzed.

Compared to earlier results for $\mathcal{C}_{\tilde{G}}$, two improvements have been realized. First, the constant term of the gravitino regeneration rate $\Gamma_{\tilde{G}}$ has been calculated consistently in the thermal field theory approach. $\mathcal{C}_{\tilde{G}}$ is obtained by integrating over $\Gamma_{\tilde{G}}$, weighted with a Fermi distribution. Second, the coefficient of the leading logarithmic term has been determined with improved accuracy. In addition, it could be demonstrated that two approaches to calculate this coefficient, one starting from individual gravitino production processes and the other using the thermal version of the optical theorem, deliver analytically the same result.

With these results predictions based on the numerical value of the gravitino abundance become significantly more reliable. This holds in particular for the next-to-leading constant term, because for the real value of the strong coupling, the leading logarithm is actually quite small, numerically only a third of the constant term. The fact that for the coefficient of the leading logarithmic term the same value has been obtained with two different methods provides a good consistency check.

As for the constant term in $\Gamma_{\tilde{G}}$, an earlier result for the contribution from soft gluons by [13] has been confirmed up to a factor of 32 which had been overlooked in [13]. To this purpose the gravitino regeneration rate was calculated using the thermal version of the optical theorem. The imaginary part of the gravitino self energy was determined in the framework of hard thermal loop effective field theory. In this framework the logarithmic divergence arising from the exchange of massless infrared gluons exchanged in the $t$ or $u$ channel of inelastic $2 \rightarrow 2$ gravitino production processes is regularized by electric Debye screening. This effect is taken into account by a resummed gluon propagator which is used instead of the bare one. 
The contribution from hard gluons to $\Gamma_{\tilde{G}}$ has been calculated in the same way. In the calculation of the one-loop gravitino self energy a resummed gluon propagator was used as well for hard loop momenta. This represents an alternative to the method of [29] where in a similar case, the calculation of the axion production rate, the contribution from hard gauge bosons was determined directly from the matrix element of axion production, rather than from the self energy. The contribution from hard gluons to the gravitino regeneration rate exhibits a strong dependence on the gravitino energy $E$.

The coefficient of the leading logarithmic term in $\mathcal{C}_{\tilde{G}}$, which is obtained along with the constant in the self energy calculation, was calculated in a second approach summing up the contributions from the four divergent gravitino production processes. The logarithmic divergence was regularized by an ad hoc introduced thermal gluon mass. Evaluating all phase space integrations in the laboratory system allows to include a quantum statistical factor in the calculation of $\mathcal{C}_{\tilde{G}}$. This factor, which is usually set to 1 in this approach, is the quantum statistical factor for the particle which is produced along with the gravitino. If this particle is a boson, the process is Bose-enhanced. If it is a fermion, the process is Fermi-suppressed. The coefficient of the logarithm obtained with the quantum statistical factor is more accurate than the one we published in [14]. Together with the abovementioned factor of 32, the two different values for the coefficient found in [13] and in [14] are brought to agreement.

With the new value for the Boltzmann collision term $\mathcal{C}_{\tilde{G}}$ our analysis [14] was repeated, where the implications of a large baryogenesis temperature, $T_{B}=\mathcal{O}\left(10^{10} \mathrm{GeV}\right)$, on the mass spectrum of superparticles was studied. No phenomenological conclusions need to be changed, because in [14] we used a gravitino production cross section which was quite close to the new value. With the new numbers, the gravitino constraints on a large $T_{B}$ scenario have even become slightly relaxed, but this effect is overcompensated by using a smaller value for the Hubble constant, $h=0.7$, whereas in [14] we had $h=1$. It remains however possible to have a large baryogenesis temperature, $T_{B}=\mathcal{O}\left(10^{10} \mathrm{GeV}\right)$. Gravitinos may be the dominant part of dark matter. 


\section{Appendix A}

\section{Feynman Rules}

Here the Feynman rules for SUSY-QCD and for gravitinos in the full and in the effective theory are given. Majorana fermions (i.e. gluinos and gravitinos) are dealt with following [39]: for each diagram a continuous fermion flow is defined. In the Feynman rules it is denoted by a thin line with an arrow. Dirac traces are taken in the direction opposite to the fermion flow. In the fermion flow formalism each diagram can be given an unambiguous sign relative to the other diagrams of a given process, and one avoids the use of the charge conjugation matrix $C$.

Note that the fermion flow is not identical with the fermion number flow (quark and squark lines carry fermion number arrows, whereas Majorana fermion lines do not), nor is in general fermion or fermion number flow identical with the momentum direction.

\section{A.1 External particle lines}

All momenta are understood to run from the left to the right. In the effective theory for light gravitinos, external gravitinos are treated like external gluinos.

- Gluons $g(P)$ with polarization vector $\varepsilon_{\mu}(P)$ :

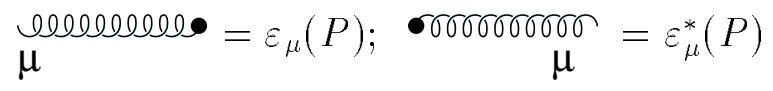

- Spin- $\frac{1}{2}$ fermions: gluinos $\tilde{g}(P)$ or quarks $q(P)$ : 


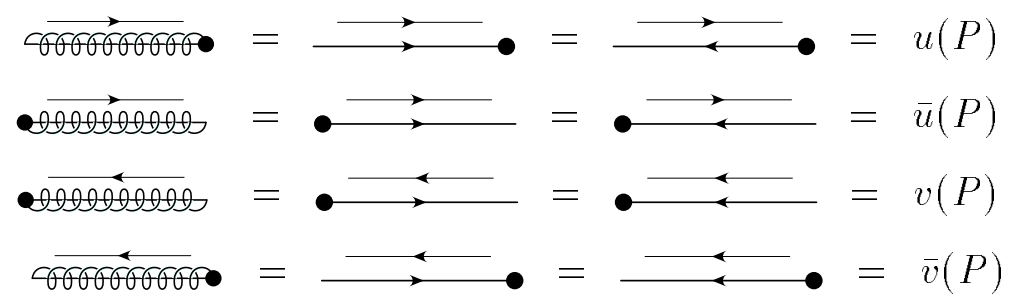

- Scalar particles: squarks $\tilde{q}(P)$ and ghosts $\eta(P)$ :

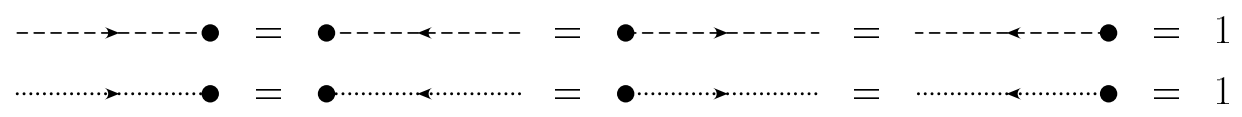

- Gravitinos $\tilde{G}(P)$ :

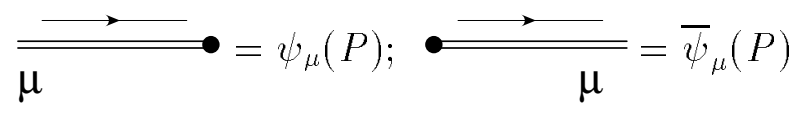

\section{A.2 Propagators}

Again, all momenta are understood to run from the left to the right.

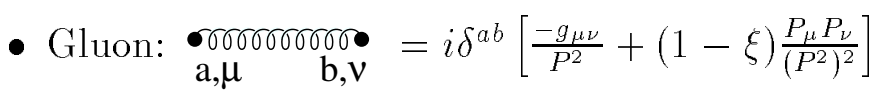

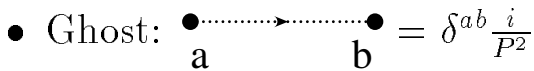

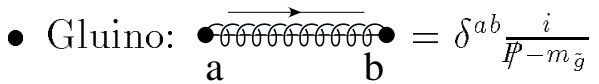

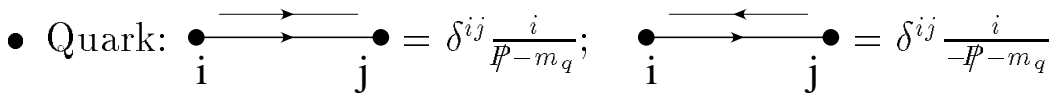

- Squark: $\stackrel{\bullet}{\mathbf{i}} \underset{\mathrm{j}}{\bullet}=\delta^{i j} \frac{i}{P^{2}-m_{\tilde{q}}^{2}}$

\section{A.3 Vertices}

Here, all momenta are understood to run into the vertex. Except for the four-squark vertex, the Feynman rules are given for only one chirality, i.e. squarks and quarks are left-handed. For the projection operators $P_{R / L}$ we have as usual $P_{R / L}=\frac{1}{2}\left(1 \pm \gamma_{5}\right)$. 


\section{A.3.1 Vertices of SUSY-QCD}

- Gluon-gluon-gluon:

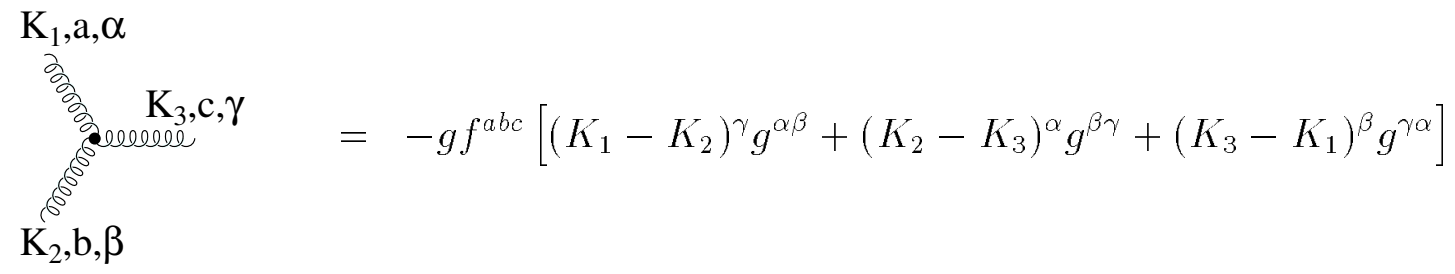

- Gluon-gluino-gluino:

a

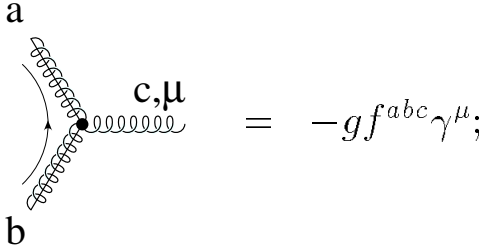

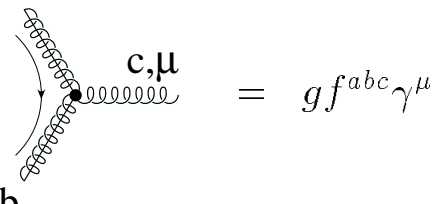

- Ghost-ghost-gluon:

$$
\underset{\mathrm{C}}{\mathrm{c}, \mu} \mathrm{P}, \mathrm{a}
$$

- Four gluons:

$$
\begin{aligned}
& \text { a, } \alpha \quad b, \beta
\end{aligned}
$$

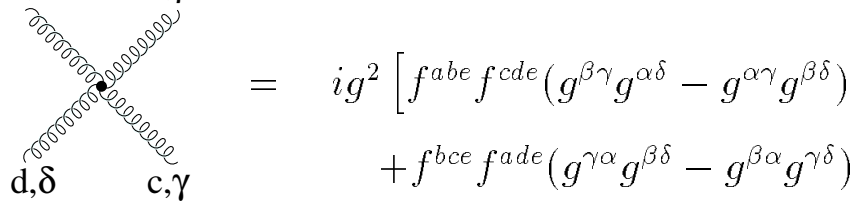

$$
\begin{aligned}
& \left.+f^{c a e} f^{b d e}\left(g^{\alpha \beta} g^{\gamma \delta}-g^{\gamma \beta} g^{\alpha \delta}\right)\right]
\end{aligned}
$$

- Quark-quark-gluon:

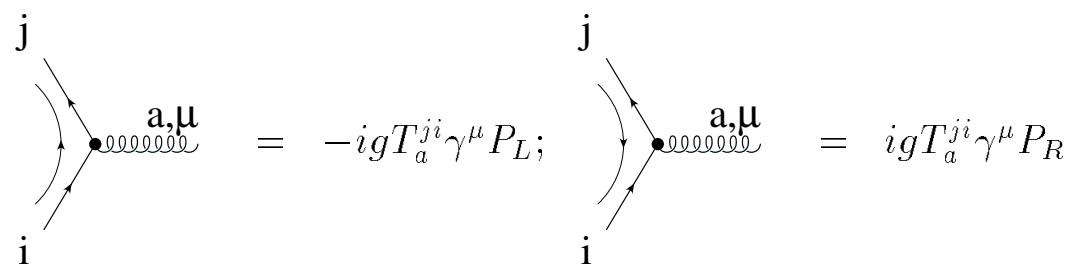


- Squark-squark-gluon:

$$
\underset{\mathrm{P}_{\mathrm{i}}, \mathrm{i}}{\mathrm{a}, \mu}
$$

- Quark-squark-gluino:
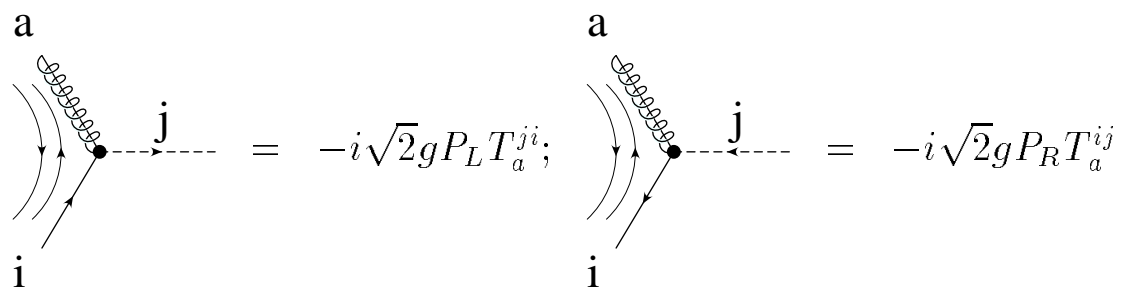

- Gluon-gluon-squark-squark:

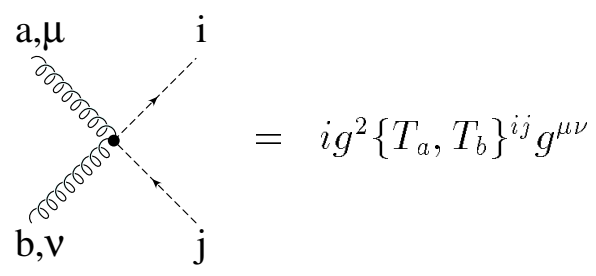

- Four squarks:

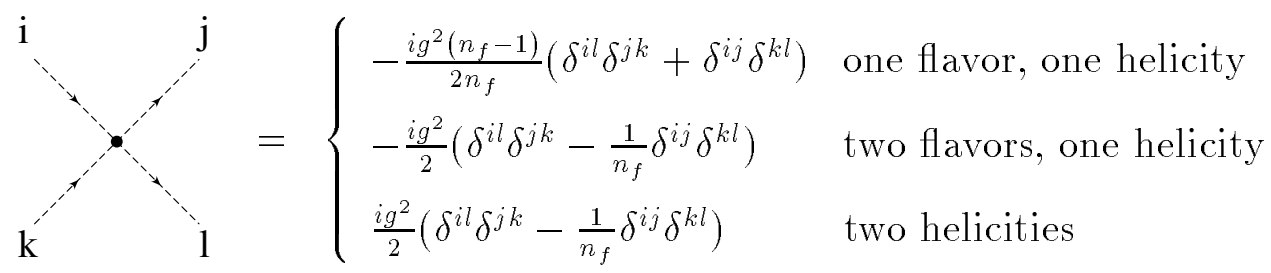

\section{A.3.2 Vertices with gravitinos (full theory)}

Gravitino vertices can be read off from the dimension-5 terms in the supergravity Lagrangian [19]:

$$
\mathcal{L}=-\frac{i}{\sqrt{2} M}\left[\left(D_{\mu}^{*} \phi^{*}\right) \overline{\psi_{\nu}} \gamma^{\mu} \gamma^{\nu} P_{L} \chi-\left(D_{\mu} \phi\right) \bar{\chi} P_{R} \gamma^{\nu} \gamma^{\mu} \psi_{\nu}\right]-\frac{i}{8 M} \overline{\psi_{\mu}}\left[\gamma^{\nu}, \gamma^{\rho}\right] \gamma^{\mu} \lambda^{a} F_{\nu \rho}^{a}
$$

Here, $\chi$ denote chiral fermion fields, $\phi$ their scalar superpartners (the Lagrangian is given for only one chirality), $F_{\mu \nu}^{a}$ is the field strength tensor of the gauge fields $A_{\mu}^{a}$ :

$$
F_{\mu \nu}^{a}=\partial_{\mu} A_{\nu}^{a}-\partial_{\nu} A_{\mu}^{a}-g f^{a b c} A_{\mu}^{b} A_{\nu}^{c}
$$


with the gauge coupling $g, \lambda^{a}$ are the gaugino fields, $\psi^{\mu}$ is the gravitino field, and $M=$ $m_{\mathrm{Pl}} / \sqrt{8 \pi}$. The covariant derivative $D_{\mu}$ is given by

$$
D_{\mu} \phi^{i}=\left(\partial_{\mu} \delta^{i j}+i g T_{i j}^{a} A_{\mu}^{a}\right) \phi^{j}
$$

For the gauge group $\mathrm{SU}(3)_{Q C D}$ one has the following vertices (all momenta run into the vertex):

- Gravitino-gluon-gluino:

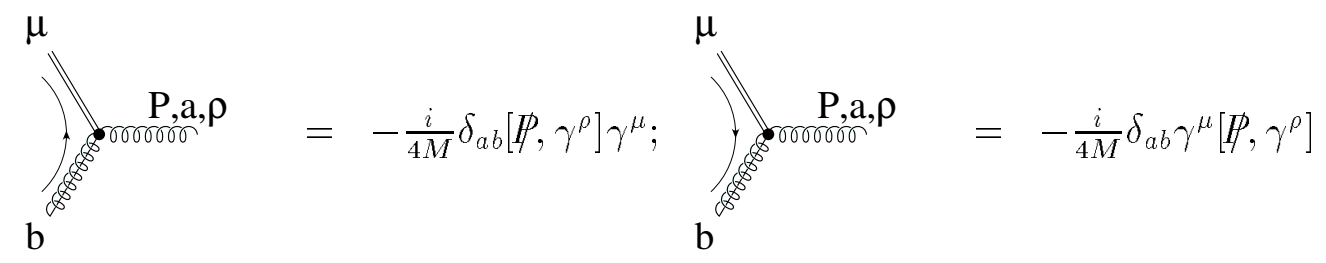

- Gravitino-gluon-gluon-gluino:
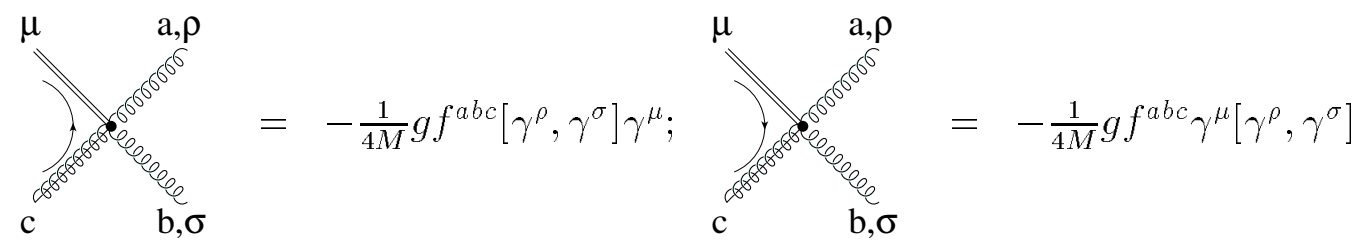

- Gravitino-quark-squark:
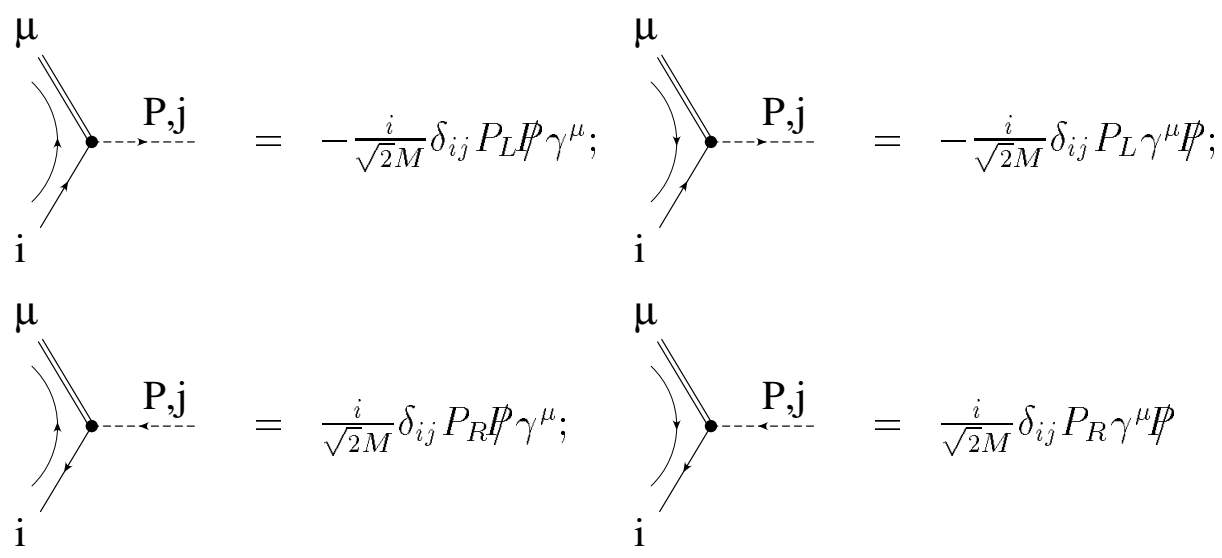
- Gravitino-quark-squark-gluon:

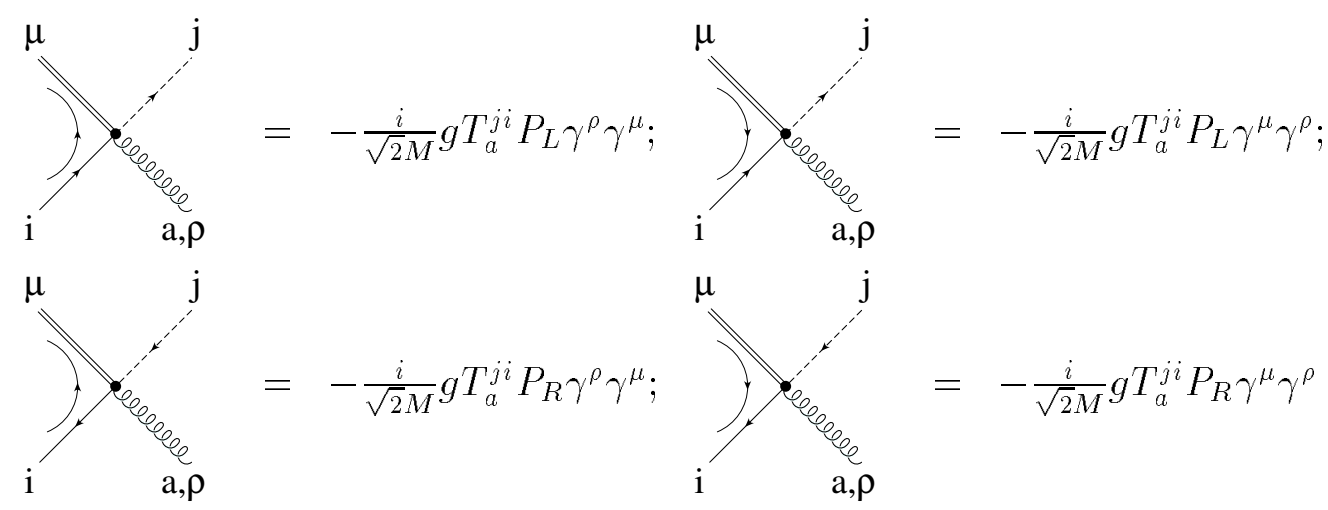

\section{A.3.3 Vertices with gravitinos (effective theory for light grav- itinos)}

These vertices can be read off from the effective Lagrangian of [22]:

$\mathcal{L}^{\mathrm{eff}}=\frac{m_{\tilde{q}}^{2}-m_{q}^{2}}{\sqrt{3} M m_{\tilde{G}}}\left(\bar{\psi} P_{L} \chi \phi^{*}+\bar{\chi} P_{R} \psi \phi\right)-\frac{m_{\tilde{g}}}{2 \sqrt{6} M m_{\tilde{G}}} \psi\left[\gamma^{\mu}, \gamma^{\nu}\right] \lambda^{a} F_{\mu \nu}^{a}-\frac{g m_{\tilde{g}}}{\sqrt{6} M m_{\tilde{G}}} \psi \lambda^{a} \phi_{i}^{*} \phi_{j} T_{i j}^{a}$.

Here $\psi$ is the goldstino (spin-1/2) component of the gravitino field. The remaining fields are the same as in the full theory Lagrangian eq. A.1. The effective theory contains the same vertices as the full theory, except for the gravitino-quark-squark-gluon vertex, which is missing in the effective theory. Instead, there is a new four particle vertex, the gravitino-gluino-squark-squark vertex (all momenta run into the vertex):

- Gravitino-gluon-gluino:

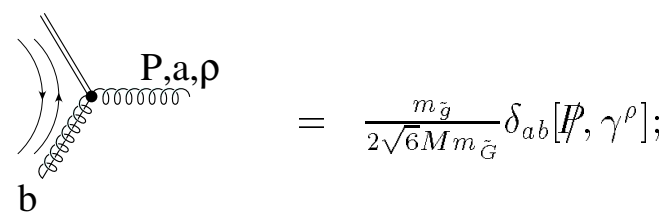

- Gravitino-gluon-gluon-gluino:

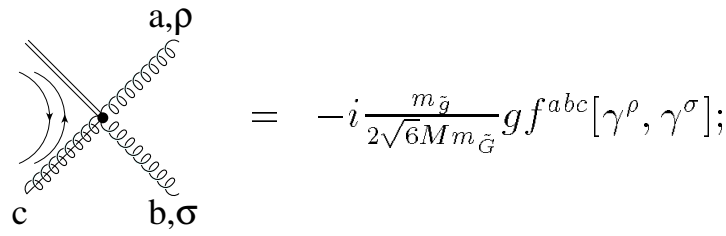


- Gravitino-quark-squark:

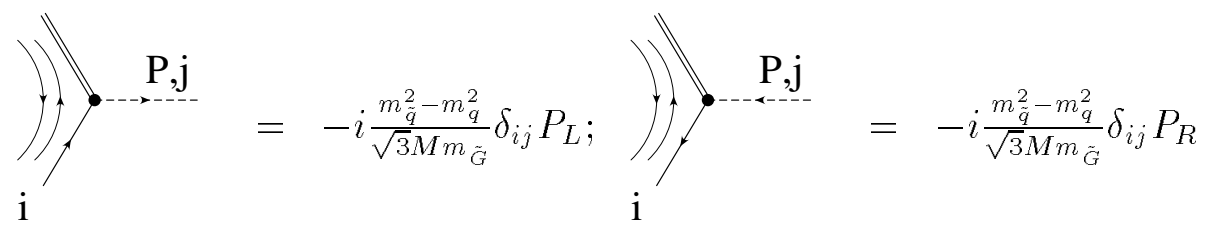

- Gravitino-gluino-squark-squark:

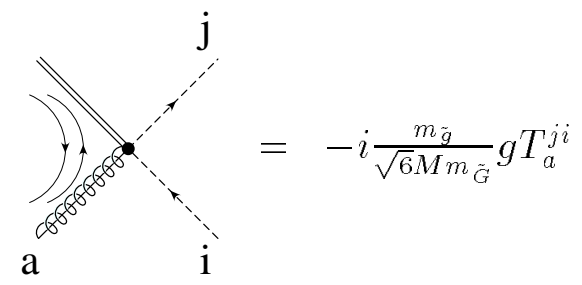




\section{Appendix B}

\section{Evaluation of The Phase Space Integrals in The Laboratory System}

When doing the phase space integrations in the Boltzmann collision term, eq. 2.23, keeping the quantum statistical factor $\left(1 \pm f_{c}^{\mathrm{Eq}}\right)$ requires to remain in the laboratory system for all integrations. In this appendix the technical details are presented which are needed in order to take into account the quantum statistical factor of a final particle in the Boltzmann collision term for a $2 \rightarrow 2$ scattering process. In the first section the general formalism is outlined. It leads to an integral expression which has to be evaluated numerically in most cases. In the second section it is shown that in the special case of the coefficient of the leading logarithmic term of $\mathcal{C}_{2 \rightarrow 2}$, the Boltzmann collision term from inelastic $2 \rightarrow 2$ gravitino production processes, this coefficient can be determined analytically in the weak coupling limit.

\section{B.1 General formalism}

The formalism will be demonstrated for massless particles, so the momenta are $P_{i}^{\mu}=$ $\left(p_{i}, \mathbf{p}_{i}\right)$. The collision term eq. 2.23 with the phase space differentials written out reads

$$
\mathcal{C}=\int \frac{d^{3} p_{a} d^{3} p_{b} d^{3} p_{c} d^{3} p_{\tilde{G}}}{16(2 \pi)^{12} p_{a} p_{b} p_{c} p_{\tilde{G}}}(2 \pi)^{4} \delta\left(P_{a}+P_{b}-P_{c}-P_{\tilde{G}}\right)|\mathcal{M}|^{2} f_{a}^{\mathrm{Eq}}\left(p_{a}\right) f_{b}^{\mathrm{Eq}}\left(p_{b}\right)\left[1 \pm f_{c}^{\mathrm{Eq}}\left(p_{c}\right)\right]
$$

The problem with this integral is that working only with the momentum three vectors of the four particles $\mathbf{p}_{a}, \mathbf{p}_{b}, \mathbf{p}_{c}$, and $\mathbf{p}_{\tilde{G}}$, one would have to deal with complicated relations for the sines and cosines of the angles between the momenta. Here it helps a lot to separate the ingoing particle side from the outgoing particle side, and it is therefore convenient to 
introduce an additional three vector $h$ representing the momentum of the center of mass of the particles in the thermal bath:

$$
\mathbf{h}=\mathbf{p}_{a}+\mathbf{p}_{b}=\mathbf{p}_{c}+\mathbf{p}_{\tilde{G}}
$$

$\mathbf{h}$ plays the role of an interface between the ingoing and outgoing particle side. Some of the angles will be measured relative to $\mathbf{h}$, and for these angles the mutual relations are much simpler.

We will need three such angles: the angle between $\mathbf{h}$ and $\mathbf{p}_{b}$, the angle between $\mathbf{h}$ and $\mathbf{p}_{c}$, and the polar angle $\varphi$ between $\mathbf{p}_{b}$ and $\mathbf{p}_{c}$ relative to the axis $\mathbf{h}$ (the three momenta of the incoming particles $\mathbf{p}_{a}$ and $\mathbf{p}_{b}$ lie in a plane $A_{i}$, and the three momenta of the outgoing particles $\mathbf{p}_{c}$ and $\mathbf{p}_{\tilde{G}}$ lie in a plane $A_{f}$. In general, $A_{i}$ and $A_{f}$ are different: they have the vector $\mathbf{h}$ in common, but around $\mathbf{h}$ they can be rotated against each other by the angle $\varphi)$. So I define

$$
\begin{aligned}
\eta & =\frac{\mathbf{h} \mathbf{p}_{b}}{h p_{b}}, \\
\eta^{\prime} & =\frac{\mathbf{h} \mathbf{p}_{c}}{h p_{c}} \\
\varphi & =\text { Polar angle between } \mathbf{p}_{b} \text { and } \mathbf{p}_{c} \text { around } \mathbf{h} .
\end{aligned}
$$

With h one can rewrite the $\delta$-function in $\mathcal{C}($ eq. B.1) as

$$
\delta\left(P_{a}+P_{b}-P_{c}-P_{\tilde{G}}\right)=\delta\left(p_{a}+p_{b}-p_{c}-p_{\tilde{G}}\right) \int d^{3} h \delta\left(\mathbf{p}_{a}+\mathbf{p}_{b}-\mathbf{h}\right) \delta\left(\mathbf{p}_{c}+\mathbf{p}_{\tilde{G}}-\mathbf{h}\right)
$$

and perform the integration over $d^{3} p_{\tilde{G}}$.

Then we write the differentials $d^{3} p_{a} d^{3} p_{b} d^{3} p_{c}$ in $\mathcal{C}$ in terms of angular variables:

$$
d^{3} p_{a} d^{3} p_{b} d^{3} p_{c}=p_{a}^{2} d p_{a} d\left(\cos \theta^{\prime}\right) d \phi_{a} p_{b}^{2} d p_{b} d(\cos \theta) d \phi_{b} p_{c}^{2} d p_{c} d \eta^{\prime} d \phi
$$

As before in eq. 2.25, $\theta$ is the angle between $\mathbf{p}_{a}$ and $\mathbf{p}_{b} . \theta^{\prime}$ and $\phi_{a}$ are the azimuthal and polar angle between $\mathbf{p}_{a}$ and some arbitrary direction of reference, and $\phi_{b}$ is the polar angle of $\mathbf{p}_{b}$ around $\mathbf{p}_{a}$. Nothing depends on $\theta^{\prime}, \phi_{a}$, and $\phi_{b}$, so they can be directly integrated over, yielding a factor of $2(2 \pi)^{2}$.

Next $\eta^{\prime}$ is eliminated by integrating over the energy $\delta$-function $\delta\left(p_{a}+p_{b}-p_{c}-p_{\tilde{G}}\right)$. This produces a factor $\frac{p_{\tilde{G}}}{p_{c} h}$ and limits the $p_{c}$ integration region: $p_{c}$ integration goes from $\frac{x-h}{2}$ to $\frac{x+h}{2}$ (with the abbreviation $x=p_{a}+p_{b}$ ).

The final step is the integration over $d^{3} h$. Now all $\delta$-functions are gone, and we are left with an integral over five independent variables: the momenta $p_{a}, p_{b}$, and $p_{c}$ and the 
angular variables $\cos \theta$ and $\varphi$ :

$$
\mathcal{C}=\frac{1}{8(2 \pi)^{6}} \int d p_{a} p_{a} f_{a}^{\mathrm{Eq}}\left(p_{a}\right) \int d p_{b} p_{b} f_{b}^{\mathrm{Eq}}\left(p_{b}\right) \int d(\cos \theta) \int_{\frac{x-h}{2}}^{\frac{x+h}{2}} d p_{c}\left[1 \pm f_{c}^{\mathrm{Eq}}\left(p_{c}\right)\right] \int d \varphi \frac{|\mathcal{M}|^{2}}{h}
$$

Here $h$ is not independent but a function of $p_{a}, p_{b}$, and $\cos \theta: h=\sqrt{p_{a}^{2}+p_{b}^{2}+2 p_{a} p_{b} \cos \theta}$. $|\mathcal{M}|^{2}$ is a function of the Mandelstam variables $s$ and $t$, therefore we need the following expressions for $s$ and $t$ in terms of the lab system variables (with $k=p_{c}-\frac{x}{2}$, and $\eta$ as a dependent variable, $\left.\eta=\frac{1}{h}\left(x-\frac{s}{2 p_{b}}\right)\right)$ :

$$
\begin{aligned}
s= & \left(P_{a}+P_{b}\right)^{2}=x^{2}-h^{2}=2 p_{a} p_{b}(1-\cos \theta) \\
t= & \left(P_{b}-P_{c}\right)^{2}= \\
& =\frac{p_{b}}{h}\left[h(h \eta-x)+2 k(x \eta-h)+2 \cos \varphi \sqrt{\left(1-\eta^{2}\right) \cdot\left(\frac{h^{2}}{4}-k^{2}\right)}\right] .
\end{aligned}
$$

The reason why in the integral eq. B.8 there are five independent variables instead of four when one works in the CMS (cf. eq. 2.24) becomes apparent here: The additional variable $\varphi$ cannot be defined in the CMS, because there $\mathbf{h}$ shrinks to zero. In the CMS, the only variable defined in terms of a quantity of the outgoing particle side is $t$, equivalent to the CMS scattering angle. In the lab system, $t$ depends on two quantities of the outgoing particle side: on $p_{c}$ and on $\varphi$. Indeed one expects five independent variables, since starting with twelve from the four momenta, four are taken away by the $\delta$-function and three correspond to the choice of the coordinate system, leaving five physical degrees of freedom.

With the quantum statistical factor $\left(1 \pm f_{c}^{\text {Eq }}\right)$ kept we obtain new quantities corresponding to the ordinary total cross section $\sigma(s)$, eq. 2.14, and the thermally averaged cross section $\Sigma^{\prime}(T)$ figuring in eq. 2.27: a thermal cross section

$$
\sigma_{\mathrm{th}}\left(p_{a}, p_{b}, \theta, T\right)=\frac{1}{64 \pi^{2} I} \int_{\frac{x-h}{2}}^{\frac{x+h}{2}} d p_{c}\left[1 \pm f_{c}^{\mathrm{Eq}}\left(p_{c}\right)\right] \int d \varphi \frac{|\mathcal{M}|^{2}}{h}
$$

and a thermally averaged cross section

$$
\Sigma(T)=\frac{\mathcal{C}}{\eta_{a} \eta_{b} n_{\mathrm{rad}}^{2}}
$$

In general, this formalism does not deliver analytic results since one has to integrate a Bose or Fermi distribution function over a finite range in the $p_{c}$ integral. It is however possible to get analytically the coefficient of the leading logarithmic term of $\mathcal{C}$ for gravitino production, as is shown in the next section. 


\section{B.2 Integration of a divergent differential cross sec- tion $\propto \frac{1}{t}$}

In order to determine the leading logarithmic term of $\mathcal{C}_{2 \rightarrow 2}$ we start from the divergent terms of the matrix elements $\left|\mathcal{M}_{i}\right|^{2}$ of table 2.1 and apply naive gluon-mass regularization, i.e. we replace $\frac{1}{t} \rightarrow \frac{1}{t-m_{\text {eff }}^{2}}$ and $\frac{1}{s+t} \rightarrow \frac{1}{s+t+m_{\text {eff }}^{2}}$. After decomposing $\left|\mathcal{M}_{F}\right|^{2}$ into partial fractions all divergent terms are $\propto \frac{s^{2}}{t-m_{\text {eff }}^{2}}$ or $\propto \frac{s^{2}}{s+t+m_{\text {eff }}^{2}}$. In the following, $\mathcal{C}$ is calculated for the first case. The second case can then be easily derived.

Substituting for $t$ the expression eq. B.10 and doing the $\varphi$ integral gives $\left(\varepsilon=m_{\mathrm{eff}}^{2} / s\right)$

$$
I_{\varphi}:=-\int d \varphi \frac{s^{2}}{t-m_{\mathrm{eff}}^{2}}=\frac{2 \pi s h}{\sqrt{\left(p_{b}-p_{c}\right)^{2}+\varepsilon\left[h^{2}(1+\varepsilon)+\left(2 p_{c}-x\right)\left(x-2 p_{b}\right)\right.}} .
$$

Without the part proportional to $f_{c}^{\mathrm{Eq}}\left(p_{c}\right)$ in the integrand of eq. B.8, one could do the $p_{c}$ integration exactly, with the result

$$
I_{c}:=\int_{\frac{x-h}{2}}^{\frac{x+h}{2}} d p_{c} I_{\varphi}=2 \pi \operatorname{sh} \ln \frac{1+\varepsilon}{\varepsilon} .
$$

For small $\varepsilon$ the main contribution to $I_{c}$ comes from the region $p_{c} \approx p_{b}$ (note that the expression in square brackets in eq. B.13 is always greater than zero). This reflects the fact that our cross section strongly enhances forward scattering. One can rigorously show that in the limit $\varepsilon \rightarrow 0$,

$$
\frac{1}{\sqrt{\left(p_{b}-p_{c}\right)^{2}+\varepsilon\left[h^{2}(1+\varepsilon)+\left(2 p_{c}-x\right)\left(x-2 p_{b}\right)\right]}} \rightarrow \delta\left(p_{b}-p_{c}\right) \ln \frac{1+\varepsilon}{\varepsilon} .
$$

With this the $p_{c}$ integration becomes trivial. All other integrations can be performed analytically.

The thermal cross section is given by

$$
\sigma_{\mathrm{th}}\left(p_{a}, p_{b}, \theta, T\right)=\frac{1}{16 \pi} \ln \frac{s+m_{\mathrm{eff}}^{2}}{m_{\mathrm{eff}}^{2}}\left[1 \pm f_{c}^{\mathrm{Eq}}\left(p_{b}\right)\right] ;
$$

it has to be compared with the total cross section $\sigma(s)=\frac{1}{16 \pi} \ln \frac{s+m_{\text {eff }}^{2}}{m_{\text {eff }}^{2}}$ obtained without the quantum statistical factor of $\left(1 \pm f_{c}^{\mathrm{Eq}}\right)$ : this factor enhances scattering for bosons and suppresses scattering for fermions, if the final state is occupied.

We use again the expansion eq. 2.28 and find after $\cos \theta$ integration

$$
\mathcal{C}=\frac{1}{16 \pi} \int \frac{d p_{a}}{2 \pi^{2}} p_{a}^{2} f_{a}^{\mathrm{Eq}}\left(p_{a}\right) \int \frac{d p_{b}}{2 \pi^{2}} p_{b}^{2} f_{b}^{\mathrm{Eq}}\left(p_{b}\right)\left[1 \pm f_{c}^{\mathrm{Eq}}\left(p_{b}\right)\right]\left(\ln \frac{1}{g^{2}}+\ldots\right) .
$$


The $p_{a}$ integral gives the equilibrium number density $\eta_{a} n_{\text {rad }}$. For the $p_{b}$ integral, two cases can occur: Both particles $b$ and $c$ are bosons (BB) or both are fermions (FF). The respective integrals give

$$
\begin{aligned}
& \mathrm{BB}: \int \frac{d p_{b}}{2 \pi^{2}} p_{b}^{2} f_{B}\left(p_{b}\right)\left[1+f_{B}\left(p_{b}\right)\right]=\frac{T^{3}}{6}, \\
& \mathrm{FF}: \int \frac{d p_{b}}{2 \pi^{2}} p_{b}^{2} f_{F}\left(p_{b}\right)\left[1-f_{F}\left(p_{b}\right)\right]=\frac{T^{3}}{12} .
\end{aligned}
$$

With $\eta_{B B}^{\prime}=1, \eta_{F F}^{\prime}=\frac{1}{2}$ we write the final form of the collision term

$$
\mathcal{C}=\frac{1}{16 \pi} \eta_{a} n_{\mathrm{rad}} \eta_{b c}^{\prime} \frac{T^{3}}{6}\left(\ln \frac{1}{g^{2}}+\ldots\right)
$$

Compared to the collision term $\mathcal{C}^{\prime}$ obtained without the quantum statistical factor, eq. 2.26, including the factor of $\left(1 \pm f_{c}^{\mathrm{Eq}}\right)$ yields a factor of $\eta_{b c}^{\prime} \frac{T^{3}}{6}$, with fermions only contributing half the amount of bosons, instead of $\eta_{b} n_{\mathrm{rad}} \approx 0.122 \eta_{b} T^{3}$, where fermions give three quarters of the boson contribution.

The collision term corresponding to a squared matrix element $|\mathcal{M}|^{2} \propto \frac{s^{2}}{s+t+m_{\text {eff }}^{2}}$ can be obtained simply by exchanging the roles of $p_{a}$ and $p_{b}$.

Before putting together the contributions from the four gravitino production processes B, $\mathrm{F}, \mathrm{G}$, and $\mathrm{H}$ to obtain the quantum statistically corrected Boltzmann collision term $\mathcal{C}_{2 \rightarrow 2}$ for gravitino production one must be sure that for the processes involving both fermions and bosons, i.e. $\mathrm{B}$ and $\mathrm{H}, t$ has been defined such that it is made up of particle momenta of the same species. This is indeed the case. 


\section{Appendix C}

\section{The Supersymmetric Effective Gluon Mass}

The standard model gluon self energy $\Pi_{\mu \nu}^{\mathrm{SM}}(K)$ of a gluon with four momentum $K=\left(k_{0}, \mathrm{k}\right)$ was first calculated by Silin, Klimov, and Weldon [30]. There are four diagrams that contribute to the one-loop order: a quark loop, a gluon loop, a ghost loop and a tadpole from the four-gluon vertex.

In the minimal supersymmetric standard model, there are three more one-loop diagrams: a gluino loop, a squark loop, and a squark tadpole from the gluon-gluon-squark-squark vertex. It turns out that the contributions of each of the four particle species gluon, gluino, quark, and squark are of the same form eq. 3.27, with individual coefficients for each particle species.

To determine the supersymmetric effective gluon mass, it is enough to look only at the zero zero component $\Pi_{00}$. The contribution to $\Pi_{00}^{\mathrm{MSSM}}$ from each of the four particle species (for technical details see e.g. [28, 40]) can be parameterized as

$$
\Pi_{00}^{i}(K)=\frac{X_{i} g^{2} T^{2}}{3}\left[\frac{k_{0}}{2 k} \ln \frac{k_{0}+k}{k_{0}-k}-1\right]
$$

with the following individual coefficients:

- $X_{\text {gluons }}=\sum_{b c}\left|f^{a b c}\right|^{2}$ from gluons,

- $X_{\text {gluinos }}=\frac{1}{2} \sum_{b c}\left|f^{a b c}\right|^{2}$ from gluinos,

- $X_{\text {quarks }}=n_{f} \sum_{i j}\left|T_{j i}^{a}\right|^{2}$ from $n_{f}$ quark flavors,

- $X_{\text {squarks }}=2 n_{f} \sum_{i j}\left|T_{j i}^{a}\right|^{2}$ from $n_{f}$ squark flavors, each with two chiralities. 
Here, $a$ is the color index of the external gluon, $b$ and $c$ are the color indices of the loop gluons or gluinos, and $i$ and $j$ are the color indices of the loop quarks or squarks.

Note that both in the chiral and in the gauge multiplet, the contribution of a boson is twice as big as the one from its fermionic partner. This corresponds to the result eq. B.20 for the Boltzmann collision term of a $2 \rightarrow 2$ gravitino production process in the LSF, where a process with a gravitino and a boson in the final state contributes twice as much as a process with a gravitino and a fermion in the final state.

Comparison with eq. 3.27 shows that the individual contributions to the effective gluon mass are $X_{i} g^{2} T^{2} / 9$. The summations are easiest done by summing over over all color degrees of freedom, and then dividing by the number of gluon color degrees of freedom $N^{2}-1$. With the relations eq. 2.29 one finds the claimed supersymmetric effective gluon mass eq. 3.28:

$$
\left(m_{g}^{\mathrm{MSSM}}\right)^{2}=\frac{g^{2} T^{2}}{6}\left(N+n_{f}\right)
$$

Compared to the standard model effective gluon mass

$$
\left(m_{g}^{\mathrm{SM}}\right)^{2}=\frac{g^{2} T^{2}}{9}\left(N+\frac{n_{f}}{2}\right)
$$

the supersymmetric mass squared is bigger by a factor of $9 / 4$ for $N=3$ and $n_{f}=6$. 


\section{Appendix D}

\section{Approximations in The Calculation of The Imaginary Part of The Gravitino Self Energy}

\section{D.1 Contribution of the pole terms to the imaginary part of the gravitino self energy}

Here it is shown that the delta function parts $\rho_{T / L}^{P}(\omega, k)$, eq. 3.36, of the gluon spectral densities $\rho_{T / L}(\omega, k)$,

$$
\rho_{T / L}^{P}(\omega, k)=\operatorname{Res}_{T / L}\left(\omega_{T / L}, k\right)\left[\delta\left(\omega-\omega_{T / L}\right)-\delta\left(\omega+\omega_{T / L}\right)\right]
$$

do not contribute to the leading logarithmic or next-to-leading constant term of the imaginary part of the gravitino self energy eq. 3.45.

The poles $\omega_{T / L}(k)$ of the transverse and longitudinal gluon propagators eq. 3.26 are solutions of transcendental equations. Their limiting forms for the two cases $k \ll m_{g}$ and $k \gg m_{g}$ are [31]:

$$
\begin{aligned}
& \omega_{T}(k)= \begin{cases}m_{g}+\frac{3}{5} \frac{k^{2}}{m_{g}}+\ldots, & k \ll m_{g}, \\
k+\frac{3}{4} \frac{m_{g}^{2}}{k}+\ldots, & k \gg m_{g},\end{cases} \\
& \omega_{L}(k)= \begin{cases}m_{g}+\frac{3}{10} \frac{k^{2}}{m_{g}}+\ldots, & k \ll m_{g}, \\
k\left(1+2 e^{-\frac{2}{3} \frac{k^{2}}{m_{g}^{2}}-2}+\ldots\right), & k \gg m_{g} .\end{cases}
\end{aligned}
$$


One can show that for all $k, m_{g}$ is a lower bound and $k+m_{g}$ is an upper bound for both poles $\omega_{T}(k)$ and $\omega_{L}(k)$. For $k \gg m_{g}$, the residues have the limiting form (I get a longitudinal residue different from [31])

$$
\begin{aligned}
\operatorname{Res}_{T}\left[\omega_{T}(k), k\right] & =\frac{1}{2 k}+\ldots, \\
\operatorname{Res}_{L}\left[\omega_{L}(k), k\right] & =-\frac{4}{3} \frac{k}{m_{g}^{2}} e^{-\frac{2}{3} \frac{k^{2}}{m_{g}^{2}}-2}(1+\ldots) .
\end{aligned}
$$

\section{Small loop momenta $k<E$}

For gluons with momenta $k<E$ in the loop, the two $\omega$ integration regions eq. 3.43 and eq. 3.44 are approximately $\omega=-k \ldots k$ and $\omega=2 E-k \ldots 2 E+k$, up to terms $\mathcal{O}\left(\frac{m_{\tilde{g}}^{2}}{E}\right)$. Therefore certainly no poles are lying within the $\omega$ integration regions for $k<m_{g}$. As for loop momenta $m_{g}<k<E$, poles will not be within the second integration region $\omega=2 E-k \ldots 2 E+k$ until $k \approx E / 3$; this case will be dealt with in the large loop momenta subsection. In order to decide whether the poles are lying within the first $\omega$ integration region one has to develop the square roots in eq. 3.43 :

$$
E-\sqrt{(p \mp k)^{2}+m_{\tilde{g}}^{2}}= \pm k-\frac{m_{\tilde{g}}^{2}}{2 E} \mp \frac{k\left(k^{2}+m_{\tilde{g}}^{2}\right)}{2 E^{2}}+\mathcal{O}\left(\frac{m_{\tilde{G}}^{2}}{E}, \frac{m_{\tilde{g}}^{4}}{E^{3}}, \frac{k^{2} m_{\tilde{g}}^{2}}{E^{3}}, \frac{k^{4}}{E^{3}}\right) .
$$

If at all, the poles $-\omega_{T / L}$ could be reached at the lower end of the integration region for $k>k_{T / L}$ with $k_{T / L}$ given approximately by the solution of the equations

$$
\begin{aligned}
\frac{m_{g}^{2}}{2 k_{T}} & =\frac{m_{\tilde{g}}^{2}}{2 E}-\frac{k_{T}^{3}}{2 E^{2}}, \\
2 k_{L} e^{-\frac{2}{3} \frac{k_{L}^{2}}{m_{g}^{2}}-2} & =\frac{m_{\tilde{g}}^{2}}{2 E}-\frac{k_{L}^{3}}{2 E^{2}} .
\end{aligned}
$$

One can exclude the pole contributions completely by requiring

$$
m_{g}^{3}>m_{\tilde{g}}^{2} E
$$

because then the right hand sides of eqs. D.7 are always negative for $k>m_{g}$. The assumption eq. D.8 is reasonable and consistent with the requirement $m_{g} \gg m_{\tilde{g}}$ which I will need in section D.2. However, if one wants to avoid assumption eq. D.8, the condition $m_{g} \gg m_{\tilde{g}}$ is sufficient to exclude the transverse pole, and the contribution from the longitudinal pole can be shown to be negligible as long as $\frac{k_{L}}{m_{g}} \gg 1$. 


\section{Large loop momenta $k>E$}

Rather than discussing under which conditions the poles are lying within the $\omega$ integration regions I will show that even if they contribute for all $k>E$ their contribution is negligible by finding an upper bound. Even if for all $k>E$ the poles contribute, their contribution to $\Gamma_{\tilde{G}}^{\text {hard }}$ cannot be larger than (cf. eq. 3.45 )

$$
\begin{aligned}
\gamma_{T / L}= & \frac{C}{8 \pi} \frac{1}{p E} \int_{E}^{\infty} d k k\left\{\left|\operatorname{Res}_{T / L} D_{T / L}\left(1+f_{\omega}-f_{q}\right)\right|_{\omega=-\omega_{T / L}(k)}+\right. \\
& \left.+\left|\operatorname{Res}_{T / L} D_{T / L}\left(f_{\omega}+f_{q}\right)\right|_{\omega=+\omega_{T / L}(k)}\right\}
\end{aligned}
$$

Note that the first integration region eq. 3.43 can only possibly cover the poles $-\omega_{T / L} \approx$ $-k$, whereas the second integration region eq. 3.44 can only possibly cover the poles $+\omega_{T / L} \approx k$. Therefore one can approximate

$$
\begin{aligned}
\left|1+f_{\omega}-f_{q}\right|_{\omega=-\omega_{T / L}(k)} & \approx\left(1+e^{-E / T}\right) e^{-k / T} \\
\left|f_{\omega}+f_{q}\right|_{\omega=\omega_{T / L}(k)} & \approx\left(1+e^{E / T}\right) e^{-k / T} .
\end{aligned}
$$

With the Dirac traces (cf. eq. 3.46, neglecting terms containing mass factors)

$$
\begin{aligned}
& \left.D_{T}\right|_{\omega= \pm \omega_{T}(k)}=\frac{9}{4} m_{g}^{4}\left(\frac{E}{k}-\frac{E^{2}}{k^{2}}\right)+\mathcal{O}\left(\frac{m_{g}^{6}}{k^{2}}\right) \\
& \left.D_{L}\right|_{\omega= \pm \omega_{T}(k)}=k^{4} e^{-\frac{2}{3} \frac{k^{2}}{m_{g}^{2}}-2}\left(1-4 \frac{E}{k}+4 \frac{E^{2}}{k^{2}}\right)+\mathcal{O}\left(k^{4} e^{-\frac{4}{3} \frac{k^{2}}{m_{g}^{2}}-4}\right),
\end{aligned}
$$

one gets

$$
\gamma_{T} \sim \mathcal{O}\left(\frac{C m_{g}^{4}}{E}\right),
$$

which is suppressed by a factor of $\frac{E^{2}}{m_{g}^{2}} \sim \varepsilon^{2}$ compared to the leading terms of $\Gamma_{\tilde{G}}^{\text {hard }}$ eq. 3.63, and a $\gamma_{L}$ which is exponentially suppressed with a factor $\sim e^{-\frac{E^{2}}{m_{g}^{2}}}$.

\section{D.2 Approximation of the $\omega$ integration limits}

Here a proof is given that the error one produces by approximating the $\omega$ integration limits to calculate the soft gluon contribution $\Gamma_{\tilde{G}}^{\text {soft }}$ to the gravitino recreation rate in section 3.2 is negligible. The proof requires the assumption that $m_{\tilde{g}} \ll m_{g}$. 
In the expression eq. 3.50 for $\Gamma_{\tilde{G}}^{\text {oft }}$, the exact integral to be calculated would read

$$
\begin{aligned}
& \int_{0}^{\frac{1}{\varepsilon}} d z \int_{x_{-}}^{x_{+}} d x\left(1-x^{2}\right)\left[\frac{z}{A_{T}^{2}+\left(z+B_{T}\right)^{2}}+\frac{2 z}{A_{L}^{2}+\left(z+B_{L}\right)^{2}}\right]=: \\
& \int_{0}^{\frac{1}{\varepsilon}} d z \int_{x_{-}}^{x_{+}} d x\left[I_{T}(x, z)+I_{L}(x, z)\right],
\end{aligned}
$$

with $A_{T / L}$ and $B_{T / L}$ given in eq. 3.35 and the $x$ integration limits (cf. eq. 3.43 )

$$
x_{ \pm}= \pm 1-\frac{m_{\tilde{g}}^{2}}{2 k E} \mp \frac{k^{2}+m_{\tilde{g}}^{2}}{2 E^{2}}+\mathcal{O}\left(\frac{m_{\tilde{G}}^{2}}{k E}, \frac{m_{\tilde{g}}^{4}}{k E^{3}}, \frac{k m_{\tilde{g}}^{2}}{E^{3}}, \frac{k^{3}}{E^{3}}\right) .
$$

In eq. 3.50, this is approximated by setting $x_{ \pm}= \pm 1$. To leading order, the integral gives a logarithm $\ln \frac{1}{\varepsilon}$ and a constant.

The corrections obtained by using the exact limits eq. D.15 will now be shown to be of higher order. To this end the $z$ integration region is subdivided into three parts: region I is from $z=0 \ldots z^{*}$, region II is from $z=z^{*} \ldots 1$, and region III is from $z=1 \ldots \frac{1}{\varepsilon}$. Here the quantity $z^{*}=k^{* 2} / m_{g}^{2}$ was introduced, where $k^{*}=m_{\tilde{g}}^{2} / m_{g}$. For loop momenta $k>k^{*}$, the corrections in the integration limits $x_{ \pm}$are smaller than $3 \varepsilon$ (note that $m_{g} / E \sim \varepsilon$ ), whereas for $k<k^{*}$ (i. e. in $z$ integration region I) they can become large. Additionally, one requires $z^{*} \ll 1$ (i. e. $m_{\tilde{g}} \ll m_{g}$ ). Note that one only has to care about corrections of $x_{ \pm}$which make the $x$ integration region smaller, since the integrand is zero for $|x|>1$.

The errors $E_{T / L}^{\mathrm{I}}, E_{T / L}^{\mathrm{II}}$, and $E_{T / L}^{\mathrm{III}}$ resulting from the three $z$ integration regions and the transverse and longitudinal term $I_{T}(x, z)$ and $I_{L}(x, z)$ in the integrand of eq. D.14 are defined as the difference between the exact expression eq. D.14 and the approximated integrals with $x_{ \pm}= \pm 1$. For these errors one can find the following upper bounds:

\section{$z$ integration region I: $z=0 \ldots z^{*}$}

As both terms $I_{T}(x, z)$ and $I_{L}(x, z)$ of the integrand in eq. D.14 are always positive or zero, integration over the whole range $x=-1 \ldots 1, z=0 \ldots z^{*}$, certainly gives an upper limit of $E_{T / L}^{\mathrm{I}}$.

Error from transverse term: For the denominator of the integrand, one has $A_{T}^{2}+$ $\left(z+B_{T}\right)^{2} \geq x^{2}+z^{2}$, therefore

$$
E_{T}^{\mathrm{I}}<\int_{0}^{z^{*}} d z \int_{-1}^{1} d x \frac{z}{x^{2}+z^{2}}<\pi z^{*}
$$

The error is $\mathcal{O}\left(z^{*}\right)$ which is below $\mathcal{O}(1)$; it is negligible. 
Error from longitudinal term: For the denominator of the integrand, one has $A_{L}^{2}+$ $\left(z+B_{L}\right)^{2}>4$, therefore

$$
E_{L}^{\mathrm{I}}<\int_{0}^{z^{*}} d z \int_{-1}^{1} d x\left(1-x^{2}\right) \frac{z}{4}=\frac{z^{* 2}}{6} .
$$

This error is even $\mathcal{O}\left(z^{* 2}\right)$ and therefore negligible, too.

\section{$z$ integration region II: $z=z^{*} \ldots 1$}

In region II and region III the corrections to the integration limits $x_{ \pm}= \pm 1$ are small, of order $\varepsilon$, and therefore one can develop the integrands around $x= \pm 1$. Around both $x=1-u$ and $x=-1+u$, for small $u$ the integrands are

$$
\begin{aligned}
& I_{T}(u, z)=2 u z\left[\left(\frac{3}{4} \pi\right)^{2}+\left(z+\frac{3}{4 u}\right)^{2}\right]^{-1}+\mathcal{O}\left(u^{2} z\right), \\
& I_{L}(u, z)=4 u z\left[\left(\frac{3}{2} \pi\right)^{2}+\left(z+3-\frac{3}{2} \ln \frac{2}{u}\right)^{2}\right]^{-1}+\mathcal{O}\left(u^{2} z\right) .
\end{aligned}
$$

Error from transverse term: $I_{T}(u, z)$ is bounded from above by $u z$, therefore

$$
E_{T}^{\mathrm{II}}<2 \int_{0}^{1} d z \int_{0}^{3 \varepsilon} d u u z=\frac{9}{2} \varepsilon^{2}
$$

(the factor of 2 is for the two borders around $x= \pm 1$ ). The error is of higher order than $\mathcal{O}(1)$

Error from longitudinal term: $I_{L}(u, z)$ is bounded from above by $u z$, too; therefore one obtains the same estimate for $E_{L}^{\mathrm{II}}$ as for $E_{T}^{\mathrm{II}}$ :

$$
E_{L}^{\mathrm{II}}<\frac{9}{2} \varepsilon^{2}
$$

$z$ integration region III: $z=1 \ldots \frac{1}{\varepsilon}$

Error from transverse term: Here one can use the upper bound $\frac{2 u}{z}$ for $I_{T}(u, z)$ (eq. D.18):

$$
E_{T}^{\mathrm{III}}<2 \int_{1}^{\frac{1}{\varepsilon}} d z \int_{0}^{3 \varepsilon} d u 2 \frac{u}{z}=18 \varepsilon^{2} \ln \frac{1}{\varepsilon}
$$

Since $\varepsilon \ln \frac{1}{\varepsilon}<\sqrt{\varepsilon}$, this error is below $\mathcal{O}\left(\varepsilon^{\frac{3}{2}}\right)$ and hence negligible. 
Error from longitudinal term: An upper bound $I_{L}(u, z)<\frac{16 u}{z}$ is valid for $\frac{3}{2} \ln \frac{1}{u}<$ $\frac{z}{2} \Leftrightarrow u>e^{-\frac{z}{3}}$. For all values of $u$, one has the bound $I_{L}(u, z)<u z$. With $\bar{z}=3 \ln \frac{1}{3 \varepsilon}$ one has the bound for the error

$$
\begin{aligned}
E_{L}^{\mathrm{III}} & <2 \int_{1}^{\bar{z}} d z \int_{0}^{3 \varepsilon} d u u z+2 \int_{\bar{z}}^{\frac{1}{\varepsilon}} d z \int_{0}^{e^{-\frac{z}{3}}} d u u z+2 \int_{\bar{z}}^{\frac{1}{\varepsilon}} d z \int_{0}^{3 \varepsilon} d u \frac{16 u}{z}= \\
& =\frac{9}{2} \varepsilon^{2}\left(\bar{z}^{2}-1\right)+\frac{9}{4}\left(e^{-\frac{2}{3} \bar{z}}-e^{-\frac{2}{3 \varepsilon}}\right)+\frac{3}{2}\left(\bar{z} e^{-\frac{2}{3} \bar{z}}-\frac{1}{\varepsilon} e^{-\frac{2}{3 \varepsilon}}\right)+144 \varepsilon^{2}\left(\ln \frac{1}{\varepsilon}-\ln \bar{z}\right)= \\
& =\frac{81}{2} \varepsilon^{2}\left(\ln \frac{1}{\varepsilon}\right)^{2}+\mathcal{O}\left(\varepsilon^{2} \ln \frac{1}{\varepsilon}\right) .
\end{aligned}
$$

This is below $\mathcal{O}(\varepsilon)$ and hence negligible. 


\section{Bibliography}

[1] S. Ferrara, D. Z. Freedman, P. van Nieuwenhuizen, Phys. Rev. D13 (1976) 3214, for a review: P. van Nieuwenhuizen, Phys. Rep. 68 (1981) 189

[2] S. Deser, B. Zumino, Phys. Rev. Lett. 38 (1977) 1433,

E. Cremmer, B. Julia, J. Scherk, P. van Nieuwenhuizen, S. Ferrara, L. Girardello, Phys. Lett. B 79 (1978) 231

[3] P. Fayet, Phys. Lett. B 84 (1979) 421

[4] H. Pagels, J. R. Primack, Phys. Rev. Lett. 48 (1982) 223

[5] S. Weinberg, Phys. Rev. Lett. 48 (1982) 1303

[6] L. M. Kraus, Nucl. Phys. B227 (1983) 556

[7] J. Ellis, A. D. Linde, D. V. Nanopoulos, Phys. Lett. B 118 (1982) 59

[8] M. D. Khlopov, A. D. Linde, Phys. Lett. B 138 (1984) 265

[9] M. Fukugita, T. Yanagida, Phys. Lett. B 174 (1986) 45

[10] J. Ellis, J. E. Kim, D. V. Nanopoulos, Phys. Lett. B 145 (1984) 181

[11] T. Moroi, H. Murayama, M. Yamaguchi, Phys. Lett. B 303 (1993) 289;

M. Kawasaki, T. Moroi, Progr. Theor. Phys. 93 (1995) 879;

T. Moroi, Ph.D. thesis, hep-ph/9503210

[12] W. Fischler, Phys. Lett. B 332 (1994) 277

[13] J. Ellis, D. V. Nanopoulos, K. A. Olive, S.-J. Rey, Astropart. Phys. 4 (1996) 371

[14] M. Bolz, W. Buchmüller, M. Plümacher, Phys. Lett. B 443 (1998) 209

[15] R. Kallosh, L. Kovman, A. Linde, A. van Proeyen, hep-ph/9907124 
[16] V. Bargmann, E. P. Wigner, Proc. Nat. Acad. Sci. (USA) 34 (1948) 211

[17] D. Lurié, Particles and Fields, Wiley, New York, 1968

[18] W. Rarita, J. Schwinger, Phys. Rev. 60 (1941) 61

[19] E. Cremmer, S. Ferrara, L. Girardello, A. van Proeyen, Nucl. Phys. B212 (1983) 413 ,

J. Wess, J. Bagger, Supersymmetry and Supergravity, Princeton University Press, Princeton, New Jersey, 1992

[20] P. R. Auvil, J. J. Brehm, Phys. Rev. 145 (1966) 1152

[21] R. Casalbuoni, D. de Curtis, D. Dominici, F. Feruglio, R. Gatto, Phys. Lett. B 215 (1988) 313

[22] T. Lee, G.-H. Wu, Phys. Lett. B 447 (1999) 83

[23] R. Cutler, D. Sivers, Phys. Rev. D17 (1978) 196

[24] L. D. Landau, E. M. Lifschitz, Lehrbuch der theoretischen Physik, vol. IV, AkademieVerlag, Berlin, 1989

[25] E. W. Kolb, M. S. Turner, The Early Universe, Addison-Wesley, Redwood City, CA, 1990

[26] M. E. Peskin, D. V. Schroeder, An Introduction to Quantum Field Theory, AddisonWesley Publishing Company, Reading, Massachusetts, 1995

[27] H. A. Weldon, Phys. Rev. D28 (1983) 2007

[28] E. Braaten, R. D. Pisarski, Nucl. Phys. B337 (1990) 569

[29] E. Braaten, T. C. Yuang, Phys. Rev. Lett. 66 (1991) 2183

[30] V. P. Silin, Sov. Phys. JETP 11 (1960) 1136,

O. Kalashnikov, V. V. Klimov, Sov. J. Nucl. Phys. 31 (1980) 699,

V. V. Klimov, Sov. Phys. JETP 55 (1982) 199,

H. A. Weldon, Phys. Rev. D26 (1982) 1394,

H. A. Weldon, Annals Phys. 271 (1999) 141

[31] R. D. Pisarski, Physica A 158 (1989) 146 
[32] R. D. Pisarski, Nucl. Phys. B309 (1988) 476

[33] V. A. Kuzmin, V. A. Rubakov, M. E. Shaposhnikov, Phys. Lett. B 155 (1985) 36

[34] W. Buchmüller, M. Plümacher, Phys. Lett. B 389 (1996) 73;

M. Plümacher, Nucl. Phys. B 530 (1998) 207

[35] J. Ellis, G. B. Gelmini, J. L. Lopez, D. V. Nanopoulos, S. Sarkar, Nucl. Phys. B373 (1992) 399

[36] W. L. Freedman, preprint astro-ph/990976 (1999)

[37] J. Edsjö, P. Gondolo, Phys. Rev. D56 (1997) 1879

[38] P. Gondolo, private communication

[39] A. Denner, H. Eck, O. Hahn, J. Küblbeck, Nucl. Phys. B387 (1992) 467

[40] M. H. Thoma in R. C. Hwa (ed.), Quark-Gluon-Plasma 2, World Scientific Publishing Company, Singapore, 1995 


\section{Acknowledgements}

I would like to thank my supervisor Wilfried Buchmüller, who suggested this investigation, for his support and encouragement as well as for highly instructive comments and discussions.

Tilman Plehn and Michael Plümacher helped me a lot in learning the basics of theoretical particle physics when I was new in the DESY theory department. I am grateful to York Schröder, Frank Eberlein, Oliver Bär, Uli Nierste, Francesco Vissani and David Delepine for valuable advice. It is my pleasure to thank Margarete Mühlleitner for her concise instructions on how to use VEGAS.

In particular, I want to thank my office mate Robert "de Niro" Kirchner for many fruitful discussions on very varied issues. 